\title{
Developing a Novel Method of Analysis for Thiols in Sauvignon blanc.
}

\author{
By Danica Christine Carter
}

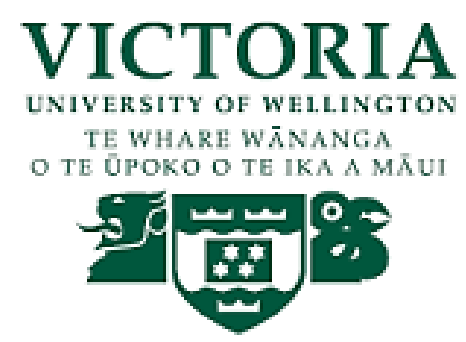

A thesis submitted to

Victoria University of Wellington

in partial fulfilment of the

requirements for the degree of

Master of Science in Chemistry.

Victoria University of Wellington

February 2014 


\section{Abstract}

New Zealand wine had an export value of $\$ 1.21$ billion in 2013. Of the total 170 million litres of wine exported that year, Sauvignon blanc made up 84.5\%. Sauvignon blanc wines have specific flavours and aromas that consumers detect and enjoy including grapefruit, passion fruit, and citrus characters that are due to the presence of sulfur containing thiols. Unfortunately, thiols are also responsible for aromas such as cat's urine, grass, and gasoline, which taint the flavour of a wine. Careful analysis of these compounds could lead to wines tailored to specific palates and a reduction of taint aromas and flavours, therefore further increasing the market potential for New Zealand Sauvignon blanc. The aim of this project was to further develop an SPME-based technique for thiol analysis of wine that is more reproducible, more accessible, and less toxic than the current method that concentrates the thiols using organomercury columns. To do this, gold nanoparticles were synthesised and coated onto SPME fibres in an attempt to selectively extract thiols from wine samples. Initial results showed an inconsistency between analyses and led to the need for a more comprehensive analysis of the gold surface, the gold-sulfur bond, and its RED-OX chemistry. Techniques employed for analysis of the gold surface included scanning electron microscopy, transmission electron microscopy, zeta-sizing and UV-Vis Spectrophotometry. To examine the interactions between gold and sulfur, Surface-Enhanced Raman Spectroscopy and computational chemistry were used. The RED-OX chemistry was initially assessed in terms of the carrier gas in the gas chromatographs but was later changed to reductive and oxidative dips. It was found that an $\mathrm{H}_{2} \mathrm{O}_{2}$ dip in between samples oxidised the bound thiolates to a series of dimers that were easier to remove from the gold. While not yet completely resolving the hysteresis observed in previous attempts, this method of cleaning the fibres will lead to future experimentation and development in this area. 


\title{
Acknowledgements
}

\author{
"When you are a Bear of Very Little Brain, and you Think of Things, \\ you find sometimes that a Thing which seemed very Thingish inside \\ you is quite different when it gets out into the open and has other people \\ looking at it."
}

- A.A. Milne.

While I may not be a Bear of Very Little Brain, this thesis is filled with Things that, without the help of my family, friends \& colleagues, would be very Thingish indeed.

I never thought I would join an organics based lab, and although I will never claim to be an organic chemist, it has been a huge learning curve, and a great experience. For this, I would like to express my gratitude to my supervisor Dr. Rob Keyzers for all of his time, his endless supply of ideas and positivity as well as his eagle eye when reading my drafts. Thanks to my lab group; Chriselle, Nathaniel, Vidhiya, Simon, Andy, Emma, Sarah, Ben and honorary lab mate Claire, thank you for putting up with the wine smells, giving me screeds of advice and making our lunch breaks such a laugh each and every day!

There were a lot of people who helped contribute to this work and to them I am very grateful; To Matthias Lein and Richard for their support in regards to computational chemistry, Andrea Kolb for her help with the characterisation of nanoparticles, To Richard Tilley for his advice and ideas in the initial stages of this project, To Eric and Brendan for all their help in the realm of physics and their expertise in SERS, To David Flynn for his time and training on the SEM and TEM and to Ben and Lucy for helping me analyse the results, To Natalie and Hannah for their help with the clean room and plasma work, To Jackie King for the use of her fume hoods and putting up with the smells in her lab too and finally to Eva and Vidhiya who started work on this project for their advice over the past two years, especially on how to get thiols out of clothing!

I would like to thank the Faculty of Science and the School of Chemical and Physical Sciences, I have truly appreciated the support and advice over the last 5 years. To those involved in the Vic Plus Leadership award, thank you for your support, opportunities to give back to the community and the skills to better prepare myself for the 'real world'. I have also very much appreciated funding from Vic Link in the initial stages of this project, the Curtis-Gordon Research Scholarship in Chemistry, and the Te Rūnanga-Ā-Iwi-O-Ngāpuhi Undergraduate Scholarship Award during my time at University.

I would very much like to thank my friends and fellow post-grads for all their support. - You have filled the past few years with great memories of BYEs (and Cavern Club), long days in the office, and far too many great (terrible) science jokes and for that I thank you all! A special thanks to Eve, Elliot and Jose, thank you for all the study sessions, crossword puzzles and horoscope readings during undergrad, it certainly made all those days writing lab reports more bearable!

Most of all, to my family who have always encouraged me. Thank you for instilling in me a love for science and a thirst for knowledge, all those family gatherings talking about chemistry, biology, physics, geology, infinity, imaginary numbers, computers, games and the likes have made me so happy to be part of the biggest family of geeks I've ever met, and thank you for making me so proud of that fact. The biggest thanks have to go to Erica, Neville and Jordy - thank you for putting up with my stressed, busy lifestyle as I got involved with everything and for always being there for me, regardless of how 'Thingish' my ideas might have seemed, I am forever grateful. 


\section{Table of Contents}

Acknowledgements. .iii

1. Introduction. 1

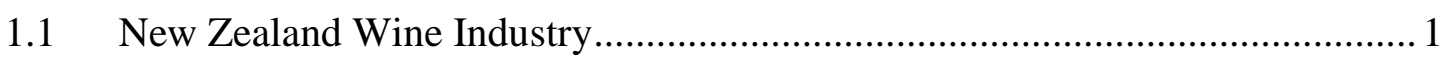

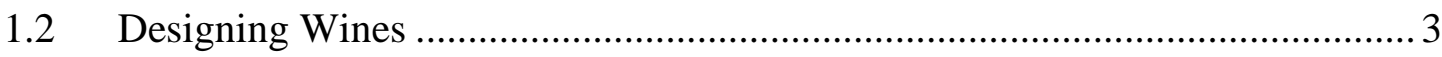

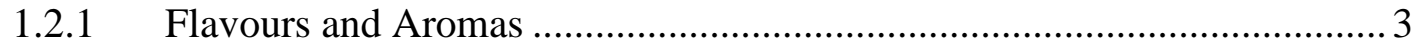

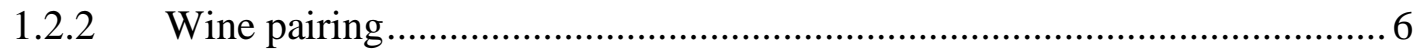

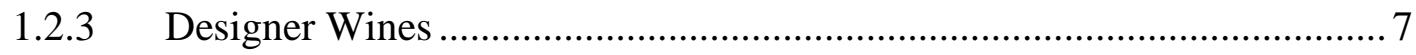

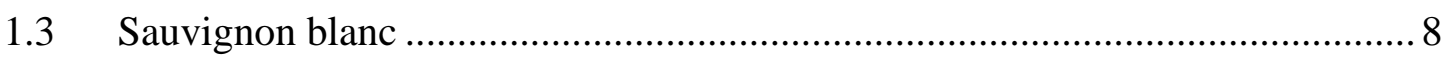

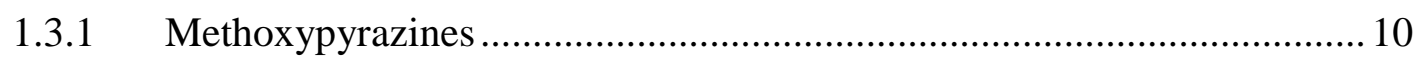

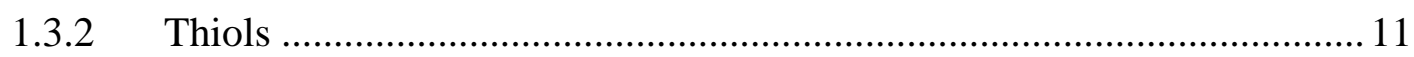

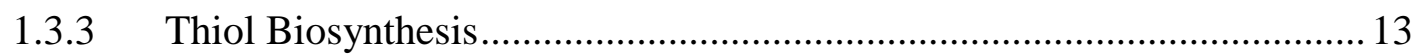

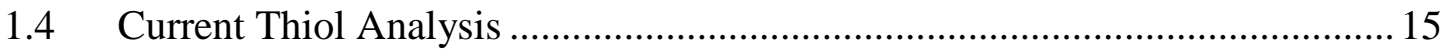

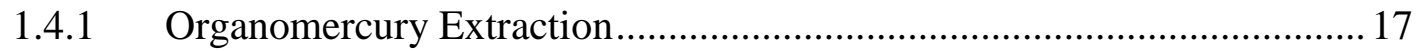

1.4.2 Thiol Analysis by Gas Chromatography............................................. 18

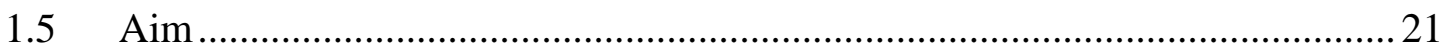

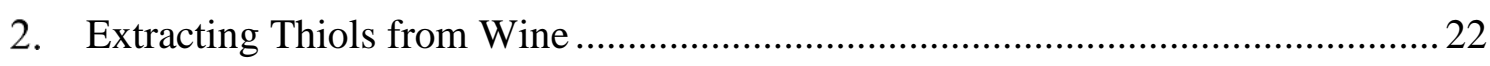

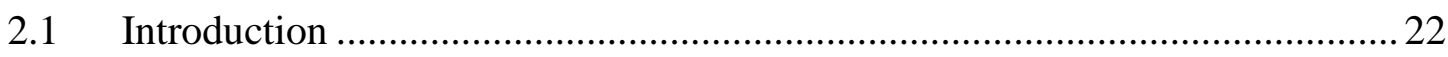

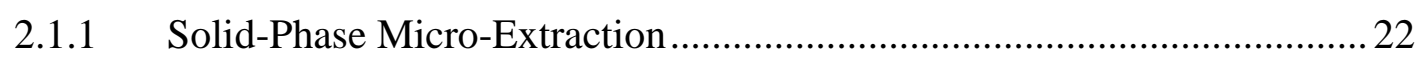

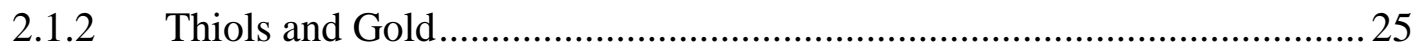

2.1.3 Kinetic and Thermodynamic Factors .................................................... 26

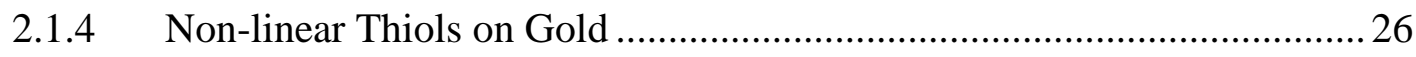

2.1.5 Gold Coated Fibre for Thiol Extraction ................................................ 27

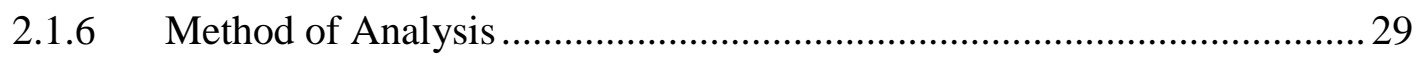

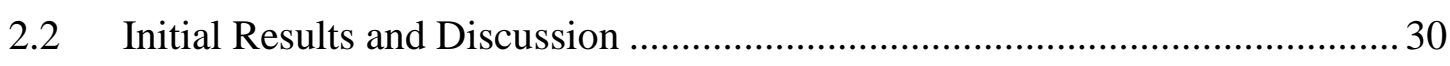

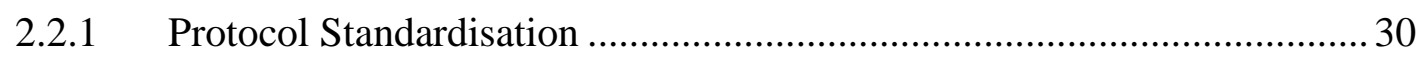

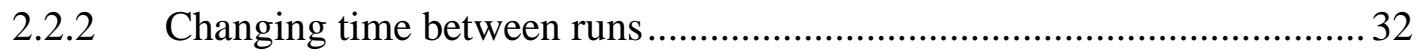

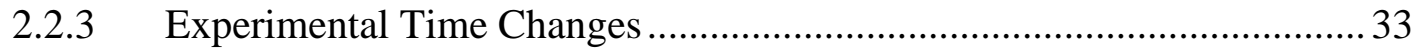

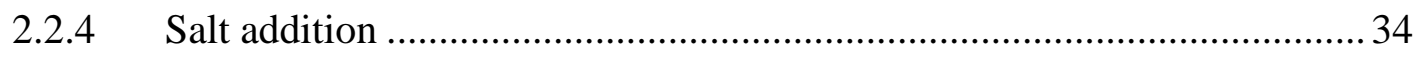

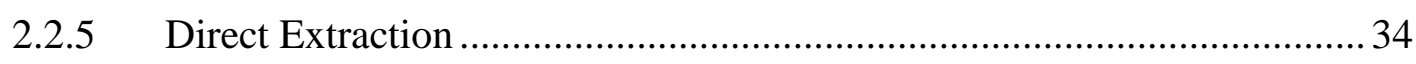

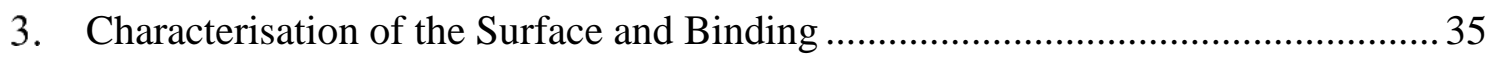

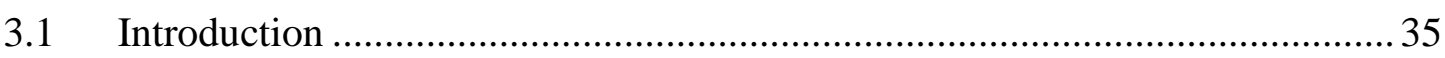

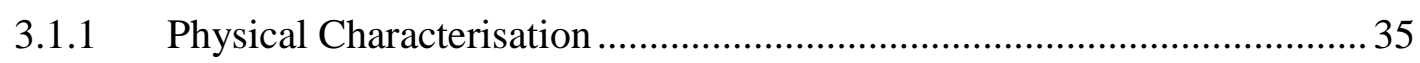

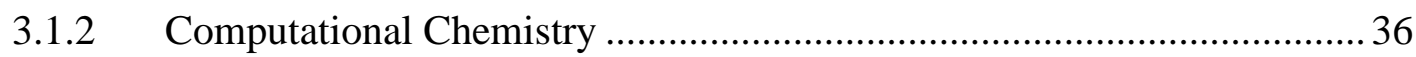

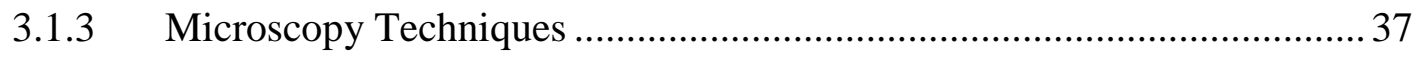


3.1.4 Surface-Enhanced Raman Spectroscopy.............................................. 38

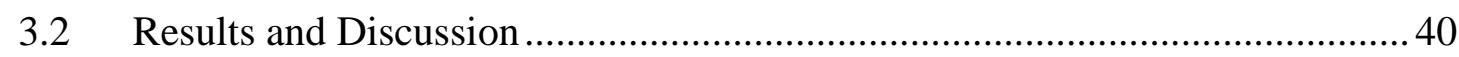

3.2.1 Microscopic and Physical Characterisation ......................................... 40

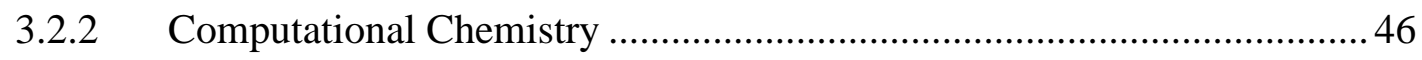

3.2.3 Surface-Enhanced Raman Spectroscopy........................................... 47

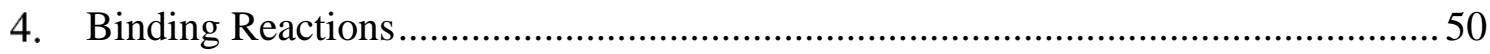

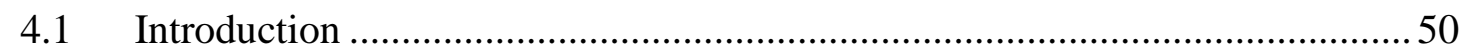

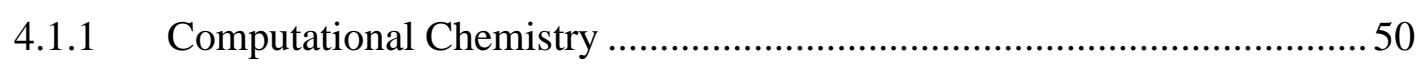

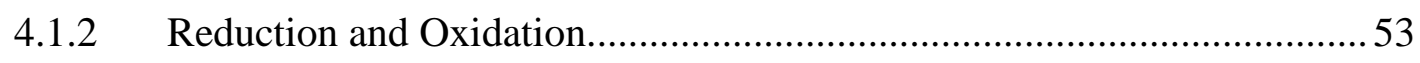

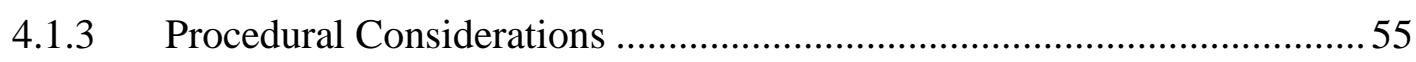

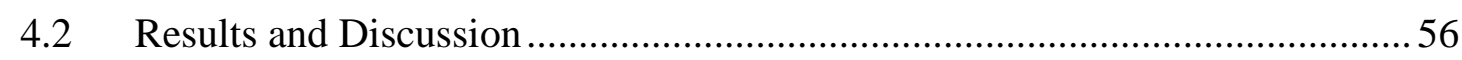

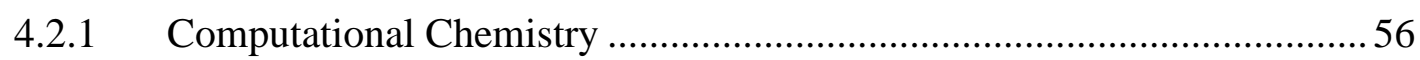

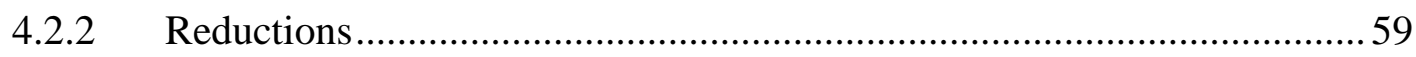

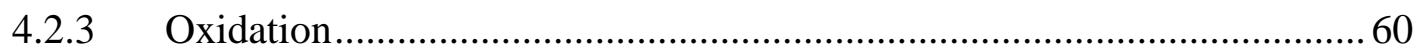

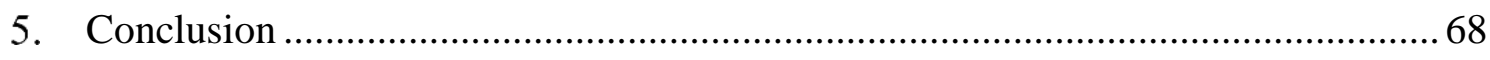

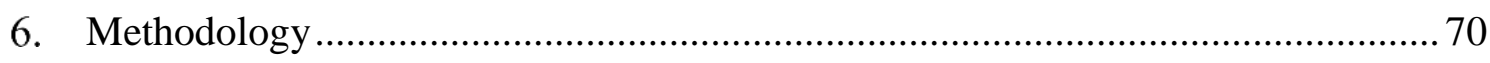

GC-MS 71

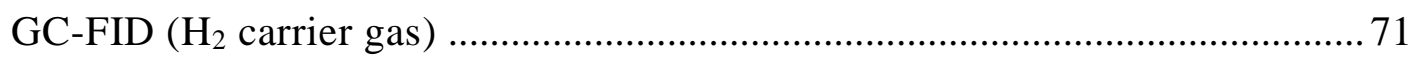

SERS 72

Plasma 72

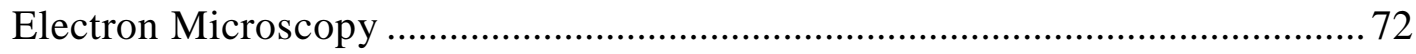

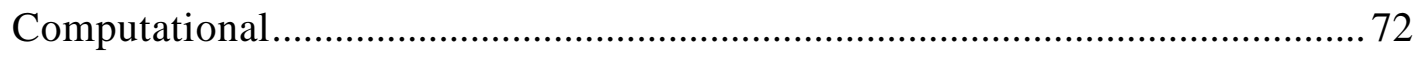

UV-Vis 73

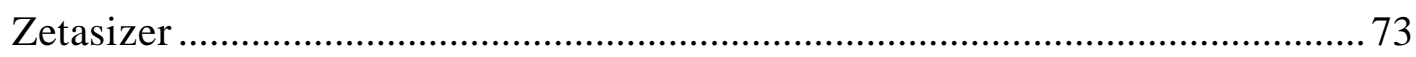

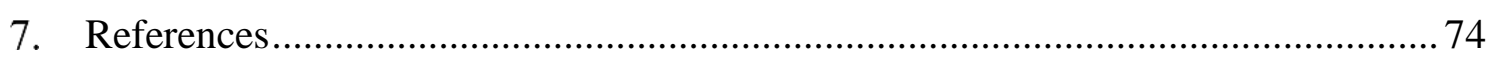




\section{Table of Abbreviations}

\begin{tabular}{|c|c|c|}
\hline 2FMT & 2-Furanmethanethiol & SAM \\
\hline 2M3FT & 2-Methyl-3-furanthiol & SBMP \\
\hline 3MSBOH & 3-Methyl-3-sulfanylbutan-1-ol & $\mathrm{sccm}$ \\
\hline 3МТP & 3-Methylthiopropanol & SEM \\
\hline $3 \mathrm{SH}$ & 3-Sulfanylhexanol & SERS \\
\hline 3SHA & 3-Sulfanylhexyl acetate & SIDA \\
\hline 4MSP & 4-Methyl-4-sulfanylpentan-2-one & SIM \\
\hline 4MSPOH & 4-Methyl-4-sulfanylpentan-2-ol & SPME \\
\hline APTMS & 3-Aminopropyltrimethoxysilane & TEM \\
\hline AMU & Atomic Mass Units & TIC \\
\hline BnT & Benzylthiol & TSC \\
\hline BP86 & Beck Perdew 86 functional & $\mathrm{TS}_{X}$ \\
\hline Cys & Cysteine & UV-Vis \\
\hline DBU & 1,8-Diazabicyclo[5.4.0]undec-7-ene & XPS \\
\hline DFT & Density Functional Theory & \\
\hline DMS & Dimethyl sulfide & \\
\hline EDS & Energy-Dispersive X-ray spectroscopy & \\
\hline EDTA & Ethylenediaminetetraacetic acid & \\
\hline FID & Flame Ionisation Detector & \\
\hline GC & Gas Chromatography & \\
\hline GSH & Glutathione & \\
\hline HS & Head Space & \\
\hline IBMP & Isobutyl-methoxypyrazine & \\
\hline IPMP & Isopropyl-methoxypyrazine & \\
\hline $\mathrm{I}_{X}$ & Intermediate & \\
\hline MQ & MilliQ triple distilled water & \\
\hline MS & Mass Spectrometry & \\
\hline $\mathrm{NCI}$ & Negative Chemical Ionisation & \\
\hline NTD & Needle Trap Device & \\
\hline $\mathrm{PFBBr}$ & Pentafluorobenzyl Bromide & \\
\hline PFPD & Pulsed-flame Photometric Detector & \\
\hline$p \mathrm{HMB}$ & $p$-Hydroxymercuribenzoate & \\
\hline PMIRRAS & $\begin{array}{l}\text { Polarisation Modulation } \\
\text { Adsorption Spectroscopy }\end{array}$ & \\
\hline RED-OX & Reduction and Oxidation & \\
\hline
\end{tabular}

Self-Assembled Monolayer

Sec-butyl-methoxypyrazine

Standard Cubic Centimetres per Minute

Scanning Electron Microscopy

Surface Enhanced Raman Spectroscopy

Stable Isotope Dilution Analysis

Selected Ion Monitoring

Solid Phase Micro Extraction

Transmission Electron Microscopy

Total Ion Chromatogram

Trisodium Citrate

Transition State

Ultra Violet and Visible

X-ray Photoelectron Spectroscopy 


\section{List of Figures}

Figure 1: 2013 Major markets by value NZ\$ (millions)......................................................... 2

Figure 2: Possible simplified odorant recognition pathway..................................................... 4

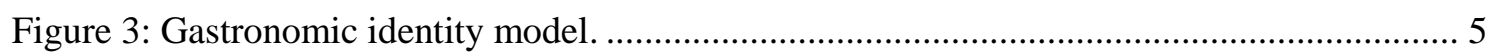

Figure 4: Volatile compounds' concentrations to predict sensory characteristics with wines by

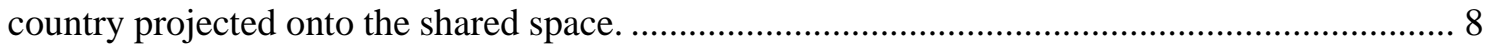

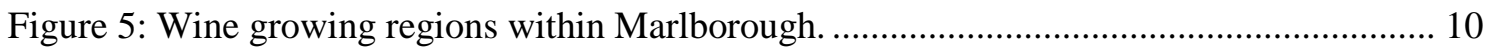

Figure 6: Structures of common methoxypyrazines in Sauvignon blanc................................... 11

Figure 7: Structures of common sulfur containing compounds in Sauvignon blanc. .................. 12

Figure 8:Representative chromatogram of an organic extract of Sauvignon blanc. .................. 16

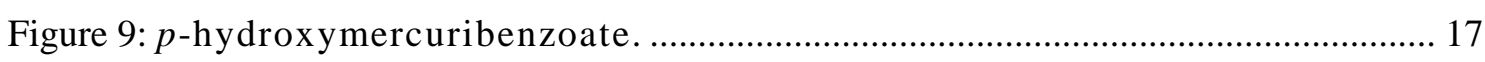

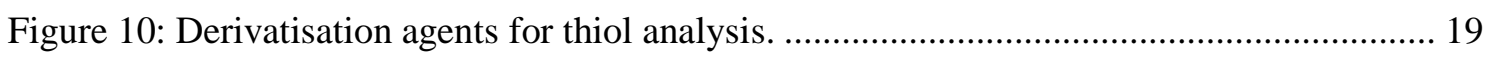

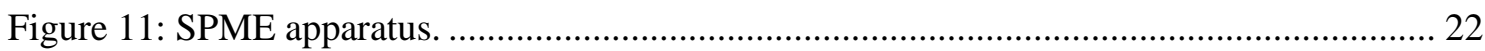

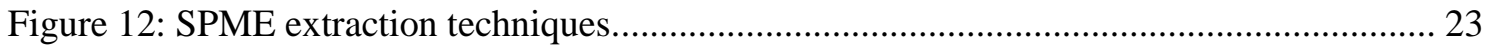

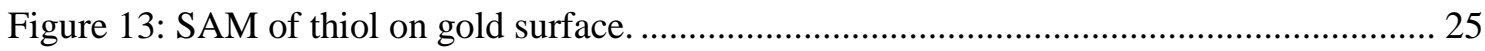

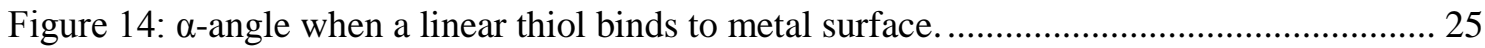

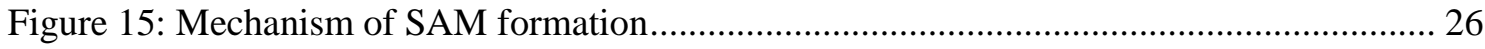

Figure 16: Side View of the orientation of 3SH and 3SHA binding to a gold surface. .............. 27

Figure 17: Uncoated (a), gold coated (b) and coated and conditioned (c) $2 \mathrm{~cm}$ fibres............... 27

Figure 18: Example of generic SPME sample preparation and GC-MS analysis...................... 29

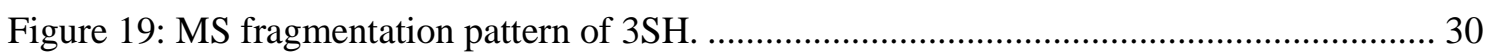

Figure 20: Overlay of HS Chromatograms of the model solution........................................... 31

Figure 21: 3SH peak area in model solution over subsequent runs. .......................................... 31

Figure 22: Peak area of 3SH after 'resting' fibre for increasing periods of time........................ 32

Figure 23: Overlay of 3SH peak heights of subsequent injections........................................... 33

Figure 24: Experimental timings in relation to the $3 \mathrm{SH}$ peak area observed.............................. 33

Figure 25: 3SHPeak areas of subsequent injections with $\mathrm{NaCl}$ additions. ................................. 34

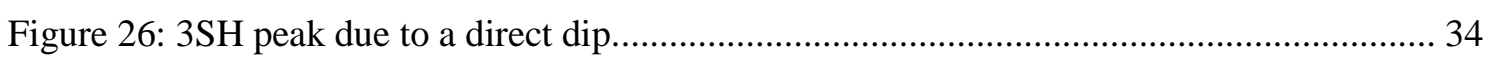

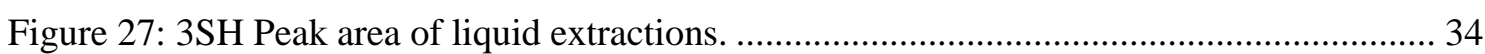

Figure 28: UV-Vis spectra for Au nanoparticles with different sizes in aqueous solution.......... 36

Figure 29: TEM diffraction pattern of gold nanoparticles ....................................................... 40

Figure 30: Concentrated (left) and diluted (right) colloidal gold samples................................. 41

Figure 31: TEM image of colloidal nanoparticles drop cast on a copper grid............................ 41

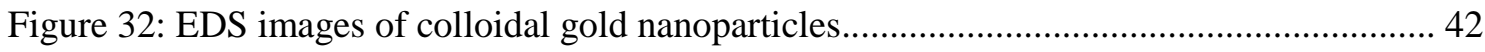

Figure 33: UV-Vis spectra of colloidal gold nanoparticle solutions........................................... 43

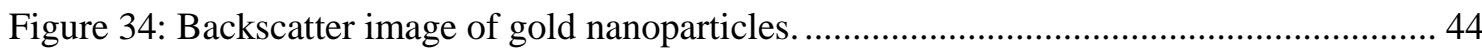




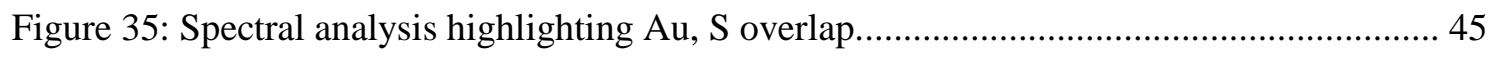

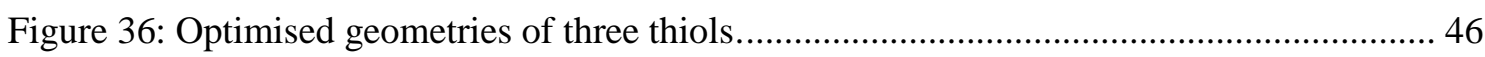

Figure 37: Predicted Raman spectra of 3SHA, 4MSP \& 3SH..................................................... 47

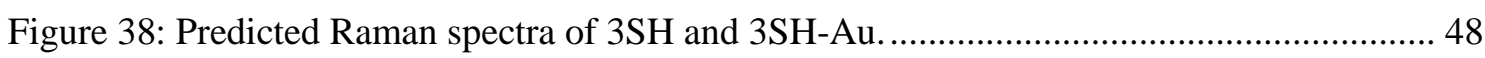

Figure 39: SERS spectra of varying concentrations of thiols in a gold colloid. .......................... 49

Figure 40: Two possible mechanisms for thiols binding to gold ................................................ 51

Figure 41: Optimised structures of reagents, products, intermediates $\left(\mathrm{I}_{\mathrm{n}}\right)$ and transition states $\left(\mathrm{TS}_{\mathrm{n}}\right)$ for the proposed mechanism of dissociative adsorption of methanethiol on a single Au........... 52

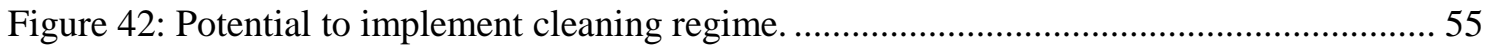

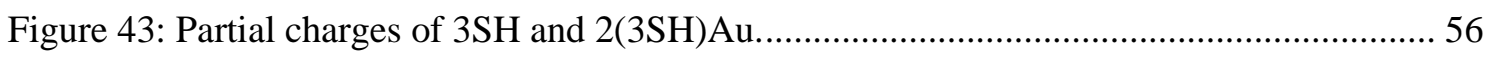

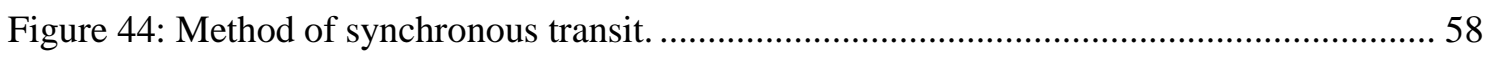

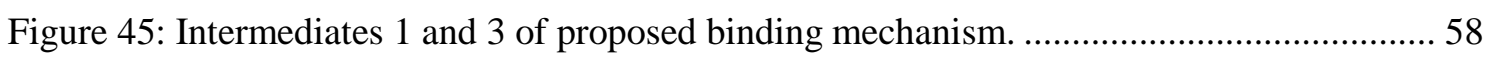

Figure 46: Peak area of 3SH over subsequent injections to the GC-FID................................... 59

Figure 47: Comparison of the effects of reductive conditions on thiol analysis using GC-MS.. 60

Figure 48: Overlay of HS Chromatograms with oxidation between each injection .................... 61

Figure 49: Overlay of HS Chromatograms with oxidation between each injection ................... 61

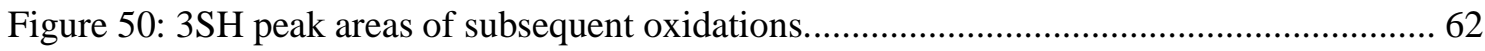

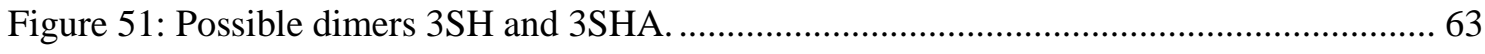

Figure 52: Mass spectral fragmentation pattern for compound eluted at 10.80 minutes ............ 64

Figure 53: Mass spectral fragmentation pattern for compound eluted at 20.25 minutes ............ 65

Figure 54: Mass spectral fragmentation pattern for compound eluted at 21 minutes. ................. 66

Figure 55: Mass spectral fragmentation pattern for compound eluted at 21.75 minutes ............. 67

\section{List of Schemes}

Scheme 1: A possible chemical pathway for the synthesis of thiols......................................... 13

Scheme 2: Possible biosynthesis pathway for thiol synthesis.................................................... 14

Scheme 3: Derivatisation scheme of the reaction of PFBBr with 3SH...................................... 20

Scheme 4: Derivatisation scheme of the reaction of N-phenylmaleimide with ethanethiol ....... 20

Scheme 5: Generic equilibria expressions for SPME adsorption ............................................... 24

Scheme 6: 3SH addition to gold, demonstrating the thiolate-gold bond. ................................... 50

Scheme 7: Possible oxidation of 3SH by $\mathrm{H}_{2} \mathrm{O}_{2}$ to form a carboxylic acid.................................... 64

Scheme 8: Fragmentation of linear thiol to give an $\mathrm{M}-\mathrm{H}_{2} \mathrm{~S}-\left(\mathrm{CH}_{2}=\mathrm{CH}_{2}\right)$ series of peaks............ 64

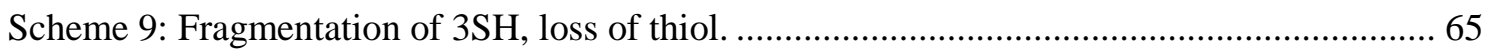




\section{List of Tables}

Table 1: New Zealand Sauvignon blanc in context with total New Zealand industry.................. 1

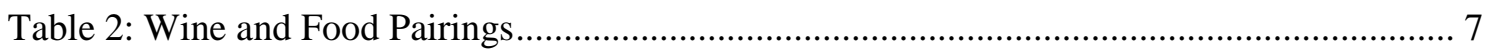

Table 3: Sulfur containing odour compounds, thresholds and perceived aroma. ....................... 12

Table 4: Compounds tentatively identified in an extracted wine sample by GC-MS ................. 16

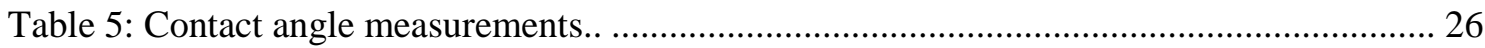

Table 6: Comparison of calculated d-spacing and reference values for gold. ............................. 40

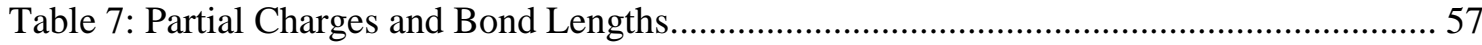




\section{Introduction}

\subsection{New Zealand Wine Industry}

The wine produced in New Zealand accounts for less than $1 \%$ of global wine production ${ }^{1}$, however, in spite of this New Zealand is currently the world's 8th largest exporter by value $^{2}$ and was ranked 11th in terms of export volume in $2012 .{ }^{1}$ This has seen a large increase in the past three years, as in 2010 New Zealand was only ranked 20th in terms of its contribution to the total volume of wine produced per year (France, Italy and Spain took the top three places respectively). ${ }^{3}$ Over the past ten years the New Zealand wine export industry has grown substantially (Table 1) from $\$ 246.4$ million in $2002^{1}$ to $\$ 1.21$ billion in $2013 .^{2}$

Table 1: New Zealand Sauvignon blanc in context with total New Zealand industry.

\begin{tabular}{cccc}
\hline New Zealand Wine Industry & $\mathbf{2 0 0 2}^{\mathbf{1}}$ & $\mathbf{2 0 1 3}^{\mathbf{2}}$ & Percentage $^{\text {growth }}$ \\
\hline Hectares of grape vines & 15800 & 35733 & 126 \\
Hectares of Sauvignon blanc vines & 4516 & 20429 & 352 \\
Grapes crushed for wine (tonnes) & 118700 & 336337 & 183 \\
Sauvignon blanc grapes crushed for wine (tonnes) & 36742 & 228781 & 523 \\
Wine exports (millions of litres) & $51.2^{*}$ & 169.7 & 331 \\
Sauvignon blanc wine exports (millions of litres) & $36.3^{*}$ & 144.6 & 298 \\
\hline
\end{tabular}

*2005 values

The past eleven years have seen a disproportionate increase in the production of Sauvignon blanc wine (Table 1). The percentage of Sauvignon blanc grape vines increased from less than $30 \%$ of the total grape vines (by area) to almost $60 \%$ and the total tonnes of Sauvignon blanc grapes approximately doubled to nearly $70 \%$ of the total tonnage. ${ }^{1,2}$ Furthermore, New Zealand imports of wine decreased by 35\% between 2002 (41.890 million litres) and 2011( 27.331 million litres). This is a significant change in the New Zealand wine trade and includes a decrease in white wine imports of approximately 10 million litres ${ }^{1}$ demonstrating that New Zealand is better able to sustain and grow within its own market. 
New Zealand's wine exports are primarily to Australia which equates to approximately $\$ 370$ million (31\% of total export value) (Figure 1). The United Kingdom imports a much greater volume of New Zealand wine, however, due to weak conversion rates, this is at a lower value, approximately $\$ 278$ million (23\% of total export value). ${ }^{2}$ The export market to the United States is reasonably comparable to that of the United Kingdom in that they have reached an export value that exceeds $\$ 250$ million and has been as an area of great opportunity in the market. Exports to Canada have seen an increase between 2011 and 2012 of approximately $20 \%$ which has seen an income of over $\$ 70$ million. A further area that has been identified as a significant opportunity for growth and income is China. These figures led to the annual wine review endorsing a focus on the markets in Asia (particularly China), North America and mainland Europe as they hold the greatest potential return for New Zealand wine. ${ }^{1}$ This trend is exaggerated by the large expansion of vineyards throughout Europe being severely restricted. Climatic and economic factors also limit Sauvignon blanc plantings in other regions of the world which clearly gives New Zealand a competitive advantage in this area. ${ }^{2}$

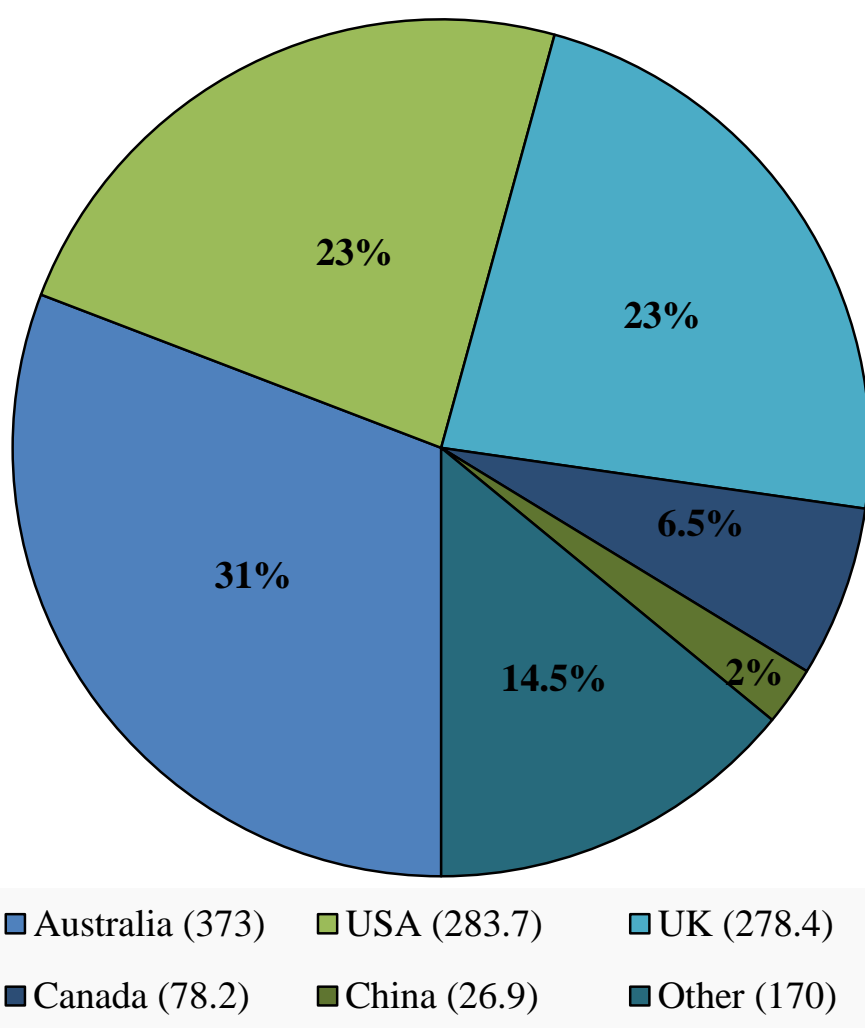

Figure 1: 2013 Major markets by value NZ\$ (millions).

From these data it is obvious that white wine in New Zealand (particularly Sauvignon blanc) is an important source of revenue for the economy. In terms of the average price at which its wines are sold, New Zealand comes in second only to France, however, New Zealand white wines tend to sell for approximately half as much as red wines (\$6.69 and $\$ 11.66$ per litre respectively. $)^{2}$ It has been suggested that the major risk New Zealand wine makers may face in the near future would be to take Sauvignon blanc for granted and fail to invest in the improvement of quality or evolve with the market. ${ }^{2}$ If white wines can be developed with a superior taste and aroma profile they could occupy higher price brackets 
or be sold in more specialised markets. Even with small increases in price per bottle there is the possibility of a great increase in revenue which could be of great economic importance.

"While there is much to be accomplished the goal is clear: To make New Zealand renowned around the world for its exceptional wines." -Stuart Smith, Chair New Zealand Winegrowers Association.

\subsection{Designing Wines}

Currently New Zealand is well known for its Marlborough Sauvignon blanc, however, a Marlborough Sauvignon blanc does not stand out from the rest of the industry. ${ }^{2}$ It is valued for its consistency as our flagship, but in a world that is craving both consistency and diversity the New Zealand wine industry must strive to produce something unique. ${ }^{2}$ A current research aim is to be able to produce wines with predicted flavours and aroma compositions, so called "designer wines". A knowledge of the relative concentrations of the odour compounds that make a wine desirable and where their origins in the wine making process are, will lead to designer wines. These wines will have specific concentrations of these favourable flavours and aromas and reduction of taints (to the preference of certain palates and markets) to increase the quality and therefore sale price of the wine.

\subsubsection{Flavours and Aromas}

Humans all have differing abilities to detect concentrations of flavours and aromas in the food and beverages that they consume. They also have personal preference in terms of what they do like and do not like, based on a combination of the detection of the compounds, their subsequent signals to the brain and the personal response exhibited. An odorant is a specific ligand that is recognised by an olfactory receptor. It has been found that most odorants are hydrophobic and a leading hypothesis on their transport is that an odorant binding protein transfers the ligand to the dendritic membrane where it is transferred to the transmembrane olfactory receptor. When the ligand binds to the receptor molecule it causes a depolarisation of the dendrite and subsequent production of an action potential from a neuron to trigger a 'smell' (Figure 2). ${ }^{4}$ 


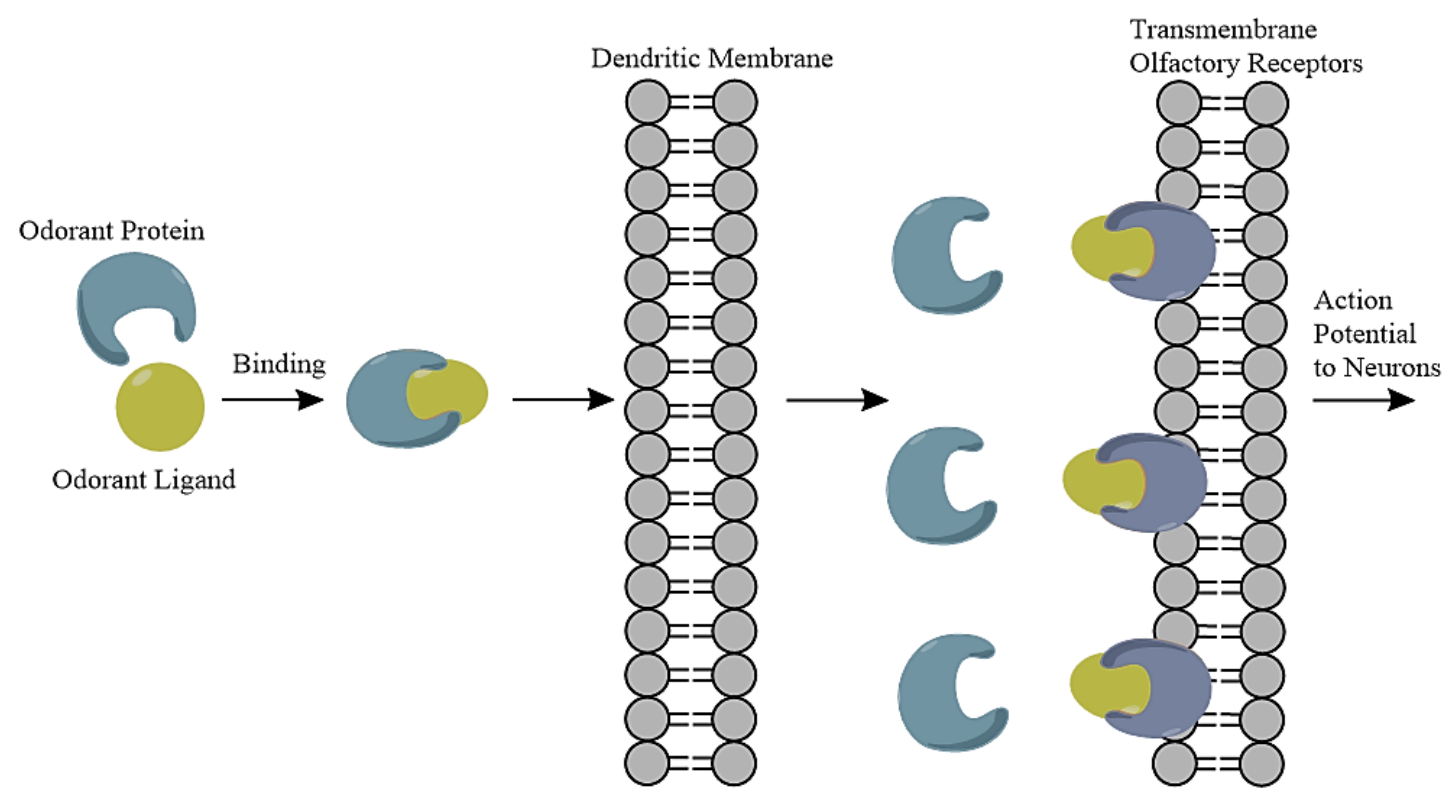

Figure 2: Possible simplified odorant recognition pathway

There are five main 'tastes' that humans can identify with their tongues; sweet, salty, bitter, sour and umami (savoury). Combinations of these tastes are what gives a food or beverage its characteristic flavour. Furthermore, these ranges of flavours have led to the development of profiles by which one can identify gastronomic preferences in both wines and food. ${ }^{5}$ While our attraction to sweet tastes appears to be innate, ${ }^{6}$ our other preferences may not be so straightforward.

Harrington et al. have examined the effects of geography and climate on regional or cultural gastronomic identity. ${ }^{5}$ They found that there are a variety of different factors that impact on what people eat and what they drink (Figure 3). They noted that throughout history, it was often seen that wine and food were consumed in and around the areas that they were produced. Knowing that the environmental factors in a region influence the agricultural products that are available to the consumers, their research indicated that the food and drink available in a region, greatly affected the flavour preferences, eating/drinking habits, recipes and dining etiquette of a culture. While the regional tastes are developing and changing with time, these progressions are based on tradition, flavour preferences, product availability and the current 'fashions'. 5 


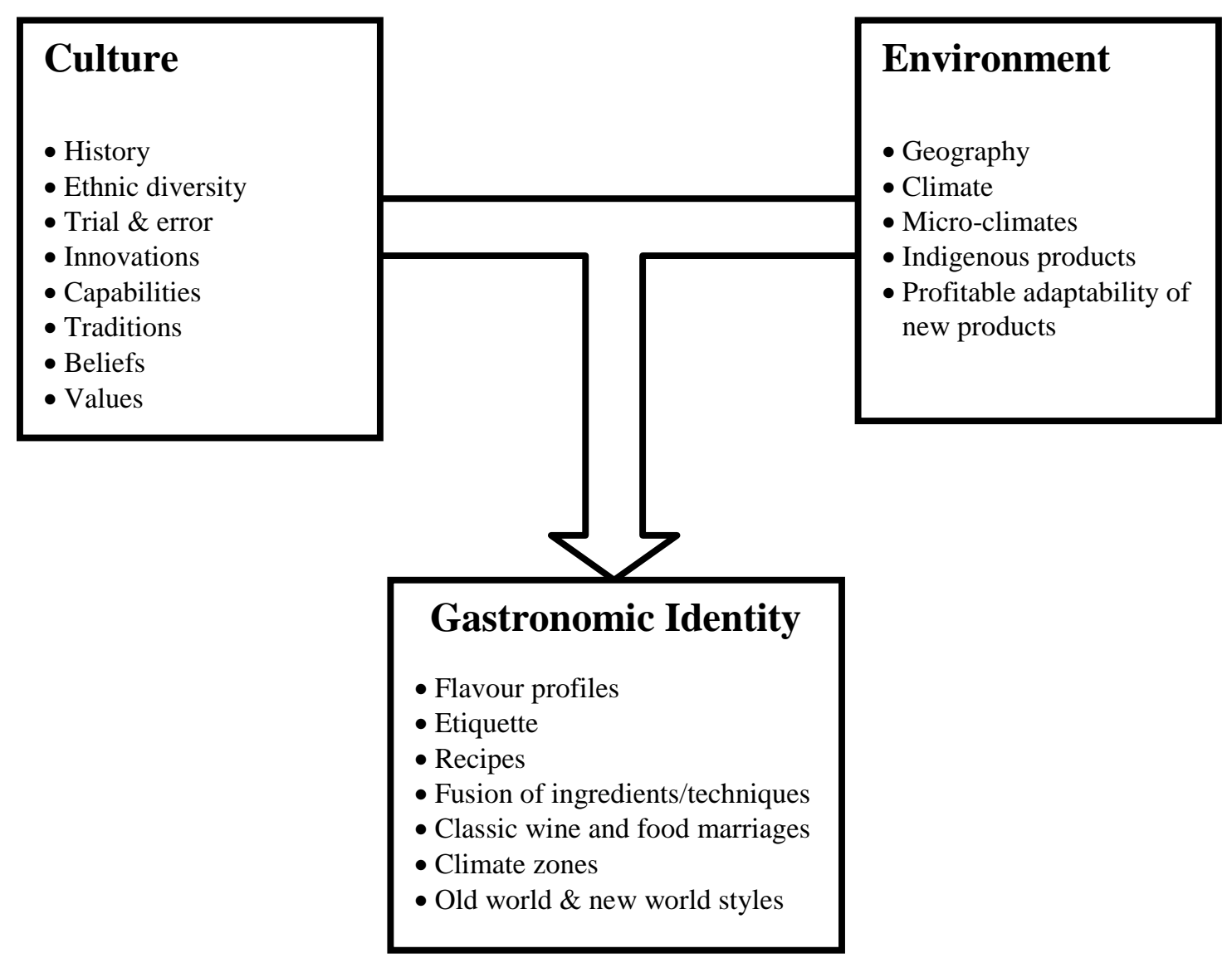

Figure 3: Gastronomic identity model. Adapted from Harrington et al. (2005)

Flavour profiles, etiquette and recipes that are unique to a region are typically derived through a continuous interaction with fashion, tradition, culture and climate as they evolve over time. The fusion of ingredients and techniques as a result of marrying diverse cultures, ethnic influences and history creates a variety of cuisines and gastronomic traditions in different regions..$^{5}$ An example of this can be seen in the generic comparison of wines made from grapes grown in cool zones and those grown in warmer zone; with all else being equal, it is often noted that the wines from cooler regions are tarter and lighter than those from the warmer regions. ${ }^{5}$ This environmental factor produces a wine that the local people become accustomed to and enjoy. This being said, it is noted that in countries like France, there is a very close relationship between the climate zones, and the type of wine-food choices that are made which indicates that pairing food with the right kind of wine is very important in the gastronomic experience. ${ }^{5}$ 


\subsubsection{Wine pairing}

Taking into consideration a culture's typical diet and preferences in terms of the five tastes (sweet, sour, bitter, salty and umami) will allow wine makers to predict possible pairing flavours for wine. While wine pairing is not an exact science, and can often depend on the personal preferences of the consumer, there are areas in which general 'rules' can be applied to get a match that will be appreciated by a large proportion of the target market. As a general rule of thumb, a white wine is typically paired with fish while a red wine complements red meat. ${ }^{7}$ This is often due to the fishy aftertaste that can be perceived in wine with fish and seafood pairing. Previous research noted that the intensity of a fishy aftertaste, due to wine and scallop pairings, was strongly correlated to the concentration of iron in the wine. ${ }^{8}$ This correlation was further supported by the observation that the intensity of fishy aftertaste in red wine increased as the researchers added ferrous ions to a model wine and when they suppressed the ferrous ion by chelation it decreased. As a final point, they found that compounds such as hexanal, heptanal, 1-octen-3-one, E,Z-2,4-heptadienal, nonanal, and decanal, (potentially potent, fishy odorant compounds) were present in dried scallops that were soaked in red wine as determined by Gas Chromatography-Olfactory (GC-O) and Gas Chromatography-Mass Spectrometry (GC-MS). Because the formations of these compounds depend on the concentration of ferrous ion in the model wine, these results suggest that ferrous ions are key to the formation of fishy aftertastes in wine and seafood pairings. ${ }^{8}$

In a more complex view of wine pairing (Table 2), it can be seen that much more complex interactions are at play. Typically a good wine match will include complementary traits, for example the weight of the wine, which then allows for contrasts, such as flavours, to work. ${ }^{7}$ Aspects of these pairings can also be seen in regions, where the climate and environment of the region 'naturally' pair wines and food in regards to what is available. Stark differences can be seen between the new world and old world wines, for example; the poor rocky soil in many old world wine countries appears to give the wines an earthy characteristic which pairs well with foods such as potatoes, truffles, cheeses, and mushrooms. Notably, this is exemplified in Spain where the earthiness of a Riojas wine pairs well with wild mushrooms in garlic oil and garlic due to the earthiness of both flavour arrangements. ${ }^{5}$ In contrast, New Zealand cuisine is a fusion of traditional European flavours and indigenous, tropical techniques that pair nicely with the high acidity and slight sweetness of a Sauvignon blanc from the same country. ${ }^{5}$ Additionally, New Zealand Sauvignon blanc is complementary to a piece of grilled fish in place of a 
squeeze of fresh lemon for acidity. ${ }^{7}$ These combinations have come about over the years after many trials and errors and this process has led to a repertoire of known pairings, and the extrapolation of expected ones.

\subsubsection{Designer Wines}

A knowledge of the flavours and aromas of specific wines and their contribution to favourable gastronomy can not only be used to break into new countries and but could be used to target sales in high end eateries both in New Zealand and overseas. For example, if a restaurant has a signature dish, an analysis could be carried out that determined the main flavours, textures and weight of the dish and compared with preferable traits in a paired wine. This in turn could then be traced back to the components responsible for these characteristics and a wine specific to the restaurant's menu could be developed therefore, increasing the diner's gastronomic satisfaction and the likelihood of positive advertisement and subsequently more business.

Table 2: Wine and Food Pairings (as described in Harrington et al. 20055)

\begin{tabular}{|c|c|c|}
\hline Wine & Food & Reasoning \\
\hline Sancerre & $\begin{array}{l}\text { Sancerrean Goat's } \\
\text { Cheese }\end{array}$ & $\begin{array}{l}\text { Acidity matches and cancels out, brings } \\
\text { out the creamy nature of the cheese and } \\
\text { smoothes both. }\end{array}$ \\
\hline Red Burgundy & Boef Bourguinon & $\begin{array}{l}\text { Red Burgundy in the Boef Bourguinon } \\
\text { sauce bridges the pair. }\end{array}$ \\
\hline Oregon Pinot Noir & $\begin{array}{l}\text { Wood-Grilled Wild } \\
\text { Salmon }\end{array}$ & $\begin{array}{l}\text { Wood-Grilling combined with the high } \\
\text { fattiness of the salmon matches the Pinot } \\
\text { Noir on a body-body level. This Pinot } \\
\text { Noir is produced in a cooler climate and } \\
\text { its subsequently higher acidity contrasts } \\
\text { nicely with the oily character of the fish. }\end{array}$ \\
\hline $\begin{array}{l}\text { Californian } \\
\text { Chardonnay }\end{array}$ & $\begin{array}{l}\text { Dungeness Crab } \\
\text { dipped in Butter }\end{array}$ & $\begin{array}{c}\text { Flavour and body match. Lower acidity of } \\
\text { the wine does not overpower the } \\
\text { sweetness of the crab }\end{array}$ \\
\hline Red Bordeaux & Lamb & $\begin{array}{l}\text { Strong texture and flavour of the lamb } \\
\text { matches the full body of the wine. Creates } \\
\text { a smooth mouth feel with the fattiness of } \\
\text { the lamb and the young Bordeaux. }\end{array}$ \\
\hline
\end{tabular}


In order to control these specific flavours and aromas, the processes by which they are biosynthesised needs to be further understood. This would require links between the biochemistry, plant physiology and biosynthesis that occurs in grapes during their growth as well as sensory science and analytical chemistry. This way, the grape berries can be analysed throughout their growth cycle and the growing conditions can be altered in order to give optimal concentrations of the specific aroma and flavour compounds for the wine. This necessitates a thorough understanding of how the grapes develop and the conditions that control the compounds formed during this process. It also requires a knowledge of the concentration at which compounds are deemed undesirable or at which they are contributing to the desirable sensory properties of a wine for a given target market.

\subsection{Sauvignon blanc}

Sauvignon blanc wine is typically very light in colour and mouth feel and is usually fermented to quite a dry level (typically fermented for longer periods of time to allow the yeast to breakdown more sugar). They are typically more acidic wines, and are therefore consumed quite soon after bottling as this is an attractive characteristic for this varietal.

Sauvignon blanc wines have specific flavours and aromas that consumers detect and enjoy. These impact odorants, which are the flavours and aromas that are distinctive to a particular varietal of wine, are volatile sulfur containing compounds (such as thiols) and methoxypyrazines, both of which are grape derived. ${ }^{9}$ The flavours and aromas of Sauvignon blanc wines tend to be characteristic of tropical fruits, passion-fruit, green capsicum, cut grass, box-tree, grapefruit and blackcurrant bud. ${ }^{10}$ A comparison between

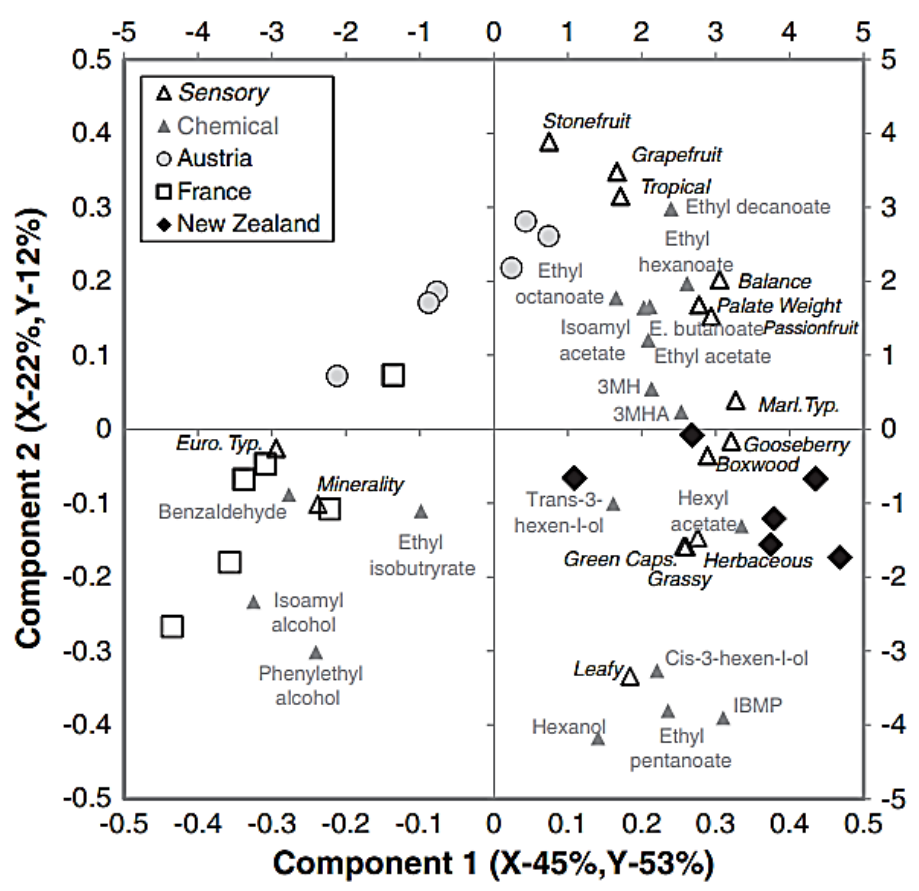

Figure 4: Volatile compounds' concentrations to predict sensory characteristics with wines by country projected onto the shared space. Reprinted from Food Research International, (44), Green, J., Parr, W., Breitmeyer, J., Valentin, D. \& Sherlock, R., Sensory and Chemical characterisation of Sauvignon blanc Wine: influence of source of origin, 2788-2797, Copyright (2011), with permission from Elsevier. 
New Zealand, French and Austrian wines presented significant differences in their flavour and aroma profiles (Figure 4). Both the New Zealand and Austrian wines contained higher concentrations of ethyl and acetate esters than the French wines which gave them fruitier/riper notes. The Austrian wines had a higher proportion of 4-methyl-4-sulfanylpentanone (4MSP) and appeared the least 'green' which is thought to be due to the low concentrations of compounds such as hexanol and cis-3-hexen-1-ol in comparison to the French wines. ${ }^{11}$ The French wines actually had high levels of compounds that, to this date, are not clearly associated with either ripe or green characteristics (e.g. ethyl isobutyrate, benzaldehyde, phenethyl alcohol and isoamyl alcohol). Sauvignon blanc wines from New Zealand (specifically Marlborough) were routinely associated with a green sensory profile (green capsicum, grassy, and leafy aromas). ${ }^{11}$ This is likely to be due to higher concentrations of compounds such as isobutyl-methoxypyrazine (IBMP), cis-3-hexen-1-ol, hexanol and trans-3-hexen-1-ol. The New Zealand wines were also found to have considerably higher concentrations 3-sulfanylhexanol and 3-sulfanylhexyl acetate (3SH \& 3SHA) which are associated with tropically fruity aromas. ${ }^{11}$ These overall results indicate that the fruity and green aromas are a combination that is a distinguishing feature of New Zealand's Marlborough Sauvignon blanc wine.

Of all the wine produced in New Zealand, the vineyards of Marlborough (located in the northeast of New Zealand's South Island) made up almost three quarters of the total grape production with 252,000 tonnes being produced per year in 2013. This completely over shadows the other regions as the second biggest contributor was Hawkes Bay with only 39,000 tonnes. Marlborough receives longer than average hours of sunlight in comparison to the rest of New Zealand, and this contributes to its 'terroir'. ${ }^{12}$ Terroir is a French oenology term which relates the environmental influence upon the chemistry of the grapes i.e. climate and geography heavily influence the concentrations of aroma compounds in the grapes and therefore the subsequent wines and their sensory properties. A wine also can be classified in terms of its typicité - representative of its terroir - as it has unique characteristics specific to its geographical area as well its chemical and physical properties. $^{13}$

It is thought that the 'green' characters in Sauvignon blanc can be manipulated through vineyard management techniques. ${ }^{14}$ A longitudinal viticultural study in New Zealand suggested that the site or location of a vineyard is a major determinant of increased concentration of IBMP and perceived green aromas. ${ }^{15}$ For example, vine attributes were 
shown to be strongly correlated to soil temperature during the summer months (particularly in January). ${ }^{16}$ It has been acknowledged that the location of vineyards in the Marlborough region also vary with respect to their terroir and subsequently the flavour and aroma profile of their respective wines. For example, Sauvignon blanc made from fruit grown in the lower Awatere Valley region were rated as much more 'Marlboroughesque' than wines from other Marlborough regions such as Rapaura, Upper Wairau, Fairhall and Brancott. ${ }^{15}$

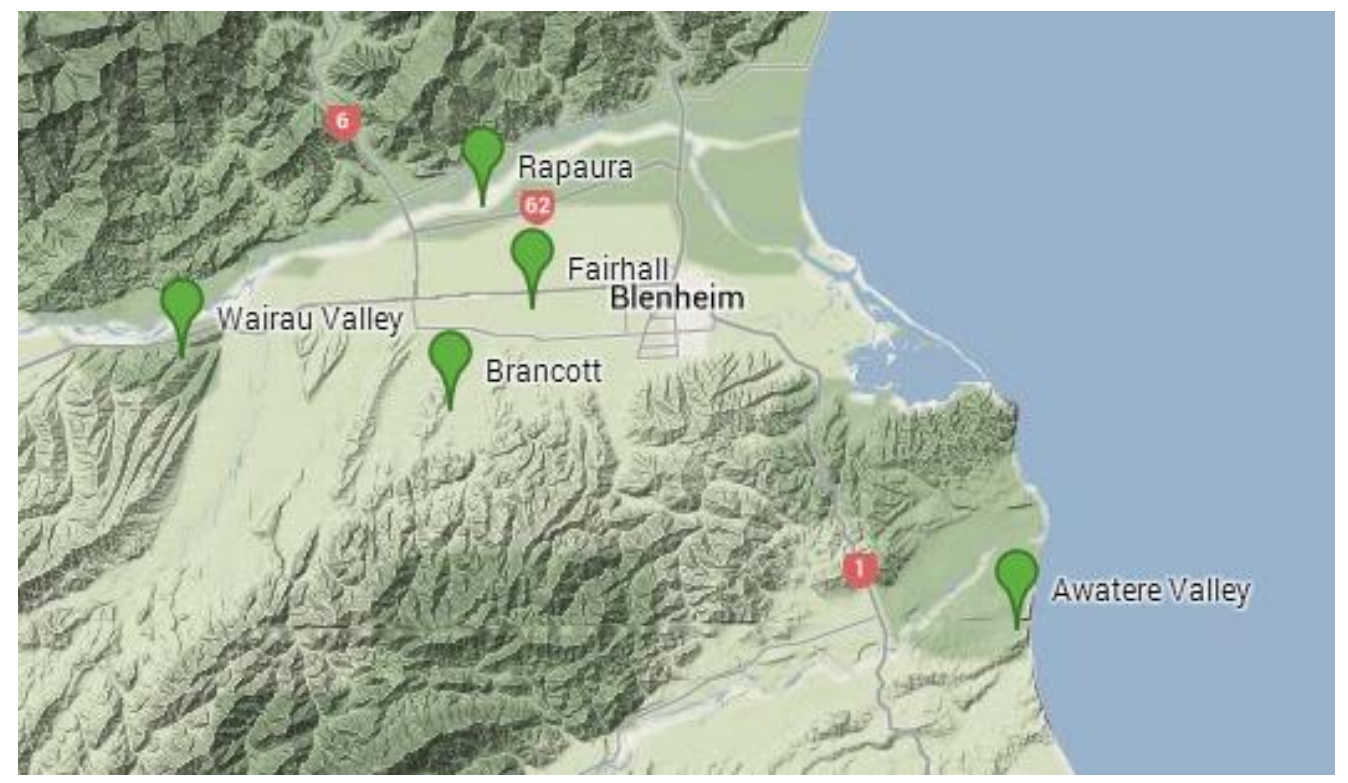

Figure 5: Wine growing regions within Marlborough.

Marlborough Sauvignon blanc wines have been preliminarily shown to be distinguishable from wines from other regions, such as France, due to the development of unique techniques for grape growing and vinification. This uniqueness evokes an odour expectation in a wine drinker that includes a perceived importance of the passion fruit, boxwood and green capsicum characteristics of the wine. This was presented in a study comparing Marlborough Sauvignon blancs produced in 2003 \& 2004 with French wines of a 2004 vintage. ${ }^{13}$ New Zealand wines appear to get their characteristic flavours and aromas from compounds such as methoxypyrazines and thiols, which leads to the need to know more about these compounds, where they originate and how to use the terroir to control their optimal production in the grapes from which they are produced.

\subsubsection{Methoxypyrazines}

Methoxypyrazines (Figure 6) are one of the most key components of Sauvignon blanc. They typically give the wine the aroma and flavour of green capsicum. The three methoxypyrazines that are the most important to wine are isopropyl-, isobutyl- and sec- 
butyl-methoxypyrazine (IPMP, IBMP \& SBMP respectively). Methoxypyrazines all have slightly different aroma descriptions, odour detection thresholds and concentrations in wine. IBMP (responsible for the green capsicum aroma) is particularly potent with an odour threshold of approximately $10-16 \mathrm{ng} \mathrm{L}^{-1}$ in wine. ${ }^{9}$ However, IBMP has been measured at levels well above its odour threshold and therefore contributes significantly to the overall aroma and flavour of the wine. ${ }^{9}$ IPMP and SBMP are also found in wine, however, they are almost always present in concentrations lower than their odour detection thresholds and are not important to the overall flavour and aroma of the wine. This is actually quite beneficial as these two compounds contribute to taints (off-flavours) such as asparagus and galbanum oil aromas. ${ }^{9}$<smiles>COc1nccnc1CC(C)C</smiles>

IBMP Isobutyl methoxypyrazine<smiles>COc1nccnc1C(C)C</smiles>

IPMP Isopropyl methoxypyrazine<smiles>CCC(C)c1nccnc1OC</smiles>

SBMP Secbutyl methoxypyrazine

Figure 6: Structures of common methoxypyrazines in Sauvignon blanc.

\subsubsection{Thiols}

Many of the sulfur containing compounds in the Sauvignon blanc wines are present as thiols, sulfides or polysulfides, most of which are detectable at the low ng $\mathrm{L}^{-1}$ level, a much lower detection threshold than many other odour compounds (Table 3). ${ }^{9}$ Thiols are organosulfur compounds that contain an R-SH functionality (the sulfur analogue of alcohols). These compounds are often referred to as mercaptans (particularly in older texts) because thiol groups bind strongly to mercury, 'capturing' them. In order to know more about the flavour and aroma contribution of these volatile thiols, it is important to measure the quantities present in varieties of wines. ${ }^{17}$ Many of these thiols contribute to the distinctive flavours and aromas in Sauvignon blanc wines, including 3SH, 3SHA and 4MSP, which give the distinctive passion fruit, citrus and guava characters. Unfortunately these compounds can also contribute to many of the 'taints' such as cooked asparagus and cat's urine. These taints are often detected when the compounds responsible are present in slightly higher concentrations than their odour detection thresholds which means that it is very important to be able to control the concentration of such compounds. ${ }^{9}$ 


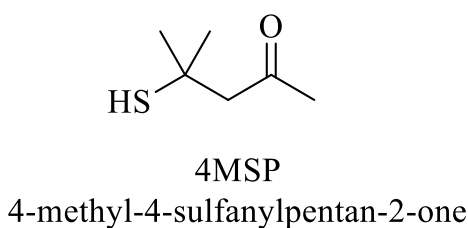

${ }^{\mathrm{OH}}$

$4 \mathrm{MSPOH}$

4-methyl-4-sulfanylpentan-2-ol<smiles>CC(C)(S)CCO</smiles>

$3 \mathrm{MSBOH}$

3-methyl-3-sulfanylbutan-1-ol<smiles>CCCC(S)CCO</smiles>

$3 \mathrm{SH}$

3-sulfanyl hexanol<smiles>CCCC(S)CCOC(C)=O</smiles>

3SHA

3-sulfanyl hexylacetate<smiles>CSC</smiles>

DMS

Dimethyl sulfide

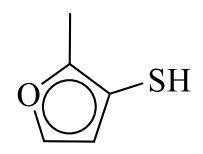

2M3FT

2-methyl-3-furanthiol

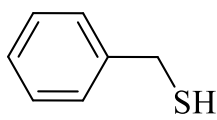

BnT

Benzylthiol

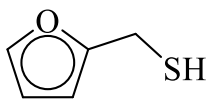

2FMS

2-Furanmethanethiol

Figure 7: Structures of common sulfur containing compounds in Sauvignon blanc.

Table 3: Sulfur containing odour compounds, thresholds and perceived aroma.

Volatile Thiol Perception Threshold

( $\mu \mathrm{g} \mathrm{L}^{-1}$ in aq alcohol)

\begin{tabular}{|c|c|c|}
\hline Volatile Thiol & $\begin{array}{l}\text { Perception Threshold } \\
\left(\mu g \mathrm{~L}^{-1} \text { in aq alcohol) }\right.\end{array}$ & Perceived Aroma \\
\hline 4MSP & 0.0008 & $\begin{array}{c}\text { Broom, citrus zest } \\
\text { urine }^{18} \text {, passion fruit } \\
\text { \& } \text { box }^{9} \text { guava } \\
\text {, } \text { cat's }^{9,10}\end{array}$ \\
\hline \multirow[t]{2}{*}{ 3SHA } & (R) 0.009 & Box tree $^{9}$, cat's urine ${ }^{9} \&$ broom ${ }^{18}$ \\
\hline & (S) 0.0025 & Passion fruit, grapefruit ${ }^{10,18}$ \\
\hline 3MSBOH & 1.5 & Cooked leeks $^{9,18}$ \\
\hline \multirow[t]{2}{*}{$3 \mathrm{SH}$} & (R) 0.005 & $\begin{array}{c}\text { Citrus, grapefruit, box tree, broom, rhubarb, cat's } \\
\text { urine, sulfur' }\end{array}$ \\
\hline & (S) 0.006 & Passion fruit, cat's urine, sulfur ${ }^{9}$ \\
\hline 4MSPOH & 0.055 & Box tree, broom, citrus zest, passion fruit ${ }^{9}$ \\
\hline $3 \mathrm{MSPOH}$ & 1000 & Sweet, potato, cauliflower, soup or meat ${ }^{9}$ \\
\hline DMS & 10 & $\begin{array}{c}\text { Black currant, cabbage, sulfur, gasoline, cooked } \\
\text { asparagus, corn, tomato }{ }^{9}\end{array}$ \\
\hline 2FMT & 0.0004 & Roasted coffee, meat, bread, popcorn ${ }^{9}$ \\
\hline 2M3FT & 0.005 & Meat $^{9}$ \\
\hline $\mathrm{BnT}$ & 0.003 & Struck match, struck flint, rubber ${ }^{9}$ \\
\hline
\end{tabular}

\section{Perceived Aroma}




\subsubsection{Thiol Biosynthesis}

It is thought that the precursors to volatile thiols are produced in the grape during berry development and growth, ${ }^{9}$ which typically proceeds via two growth periods separated by véraison (when the ripening of the berry and colour change begins to take place - pea green to yellow or purple for white and red respectively), the first of which lasts approximately 60 days from bud break. During this time, the berry swells in volume as the solutes accumulate. ${ }^{19}$ The biosynthesis of these thiols is not particularly well understood and it is possible that they can occur via several mechanisms.

\subsubsection{Addition to an Alkene}

Addition of $\mathrm{H}_{2} \mathrm{~S}$ to an alkene has been proposed as one source of thiols in wines, ${ }^{20}$ for example, the addition of $\mathrm{H}_{2} \mathrm{~S}$ to 4-methylpent-3-en-2-ol to form 4-methyl-4-sulfanylpentan-2-ol (Scheme 1). However, the non-racemic ratios of 3SHA found in wine juices are inconsistent with a purely chemical means of formation of the thiols which indicates this is not as straightforward as this purely chemical route. ${ }^{9}$

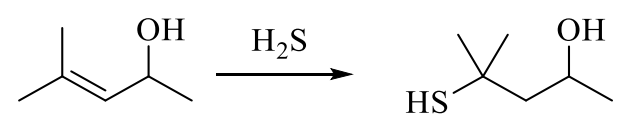

Scheme 1: A possible chemical pathway for the synthesis of thiols.

It is possible that this could be explained by enzymatic esterification of $3 \mathrm{SH}$ in which the enzyme produces one configurational isomer over the other ${ }^{10}$ or possibly a lack of sulfur donors (such as $\mathrm{H}_{2} \mathrm{~S}$ ) during the fermentation process. ${ }^{21}$ It has been discovered that by adding increasing amounts of $\mathrm{NaSH} . \mathrm{xH}_{2} \mathrm{O}$ to Sauvignon blanc grape juice prior to fermentation, the excess $\mathrm{H}_{2} \mathrm{~S}$ reacts to give increased concentrations of 3SH/A in the subsequent wine. ${ }^{21}$ However, while the increase of thiols seems to be dependent on the addition of $\mathrm{H}_{2} \mathrm{~S}$ to the grape juice, it has also been noted that there is a limit to which this is efficient in the system, either due to toxicity of $\mathrm{H}_{2} \mathrm{~S}$ or the exhaustion of reaction partners in the grape must. This was observed in the loss of efficiency in terms of the $\mathrm{H}_{2} \mathrm{~S}$ conversion to $3 \mathrm{SH} / \mathrm{A}$. When $0.00-0.12 \mu \mathrm{M}$ concentrations of $\mathrm{H}_{2} \mathrm{~S}$ were added to the grape juice there was a conversion rate of $22.3 \mathrm{pM}$ of $3 \mathrm{SH} / \mathrm{A}$ formed for every $\mathrm{nM}$ of $\mathrm{H}_{2} \mathrm{~S}$ added, whereas if $123.6-1236.2 \mu \mathrm{M}$ was used, the conversion rate was only $1.7 \mathrm{pM} .^{21}$

\subsubsection{Bound Conjugates}

Another leading hypothesis for the biosynthesis for organosulfur compounds is that nonvolatile thiol precursors, which are called bound conjugates, are found in the grape; 
cysteine and glutathione are potential conjugating groups. Cysteine is a sulfur-containing amino acid while glutathione is a tripeptide containing glutamic acid, cysteine and glycine residues. Therefore, there are two possible routes for enzymatic thiol synthesis (Scheme 2). The first is cleavage of the S-cysteine or S-glutathione bond to release the free thiol. It has been noted that the $\beta$-lyases that catalyse this cleavage have been shown to preferentially release the $S$ enantiomer which may help account for the different enantiomeric ratios noted previously. ${ }^{9}$ Scheme 2 depicts how cysteine and glutathione can bind to the alkene precursor and subsequently be cleaved to form the thiol. The alternative pathway to this is glutathione binding, followed by two peptide cleavages to afford the cysteine product which is then cleaved to afford the thiol.

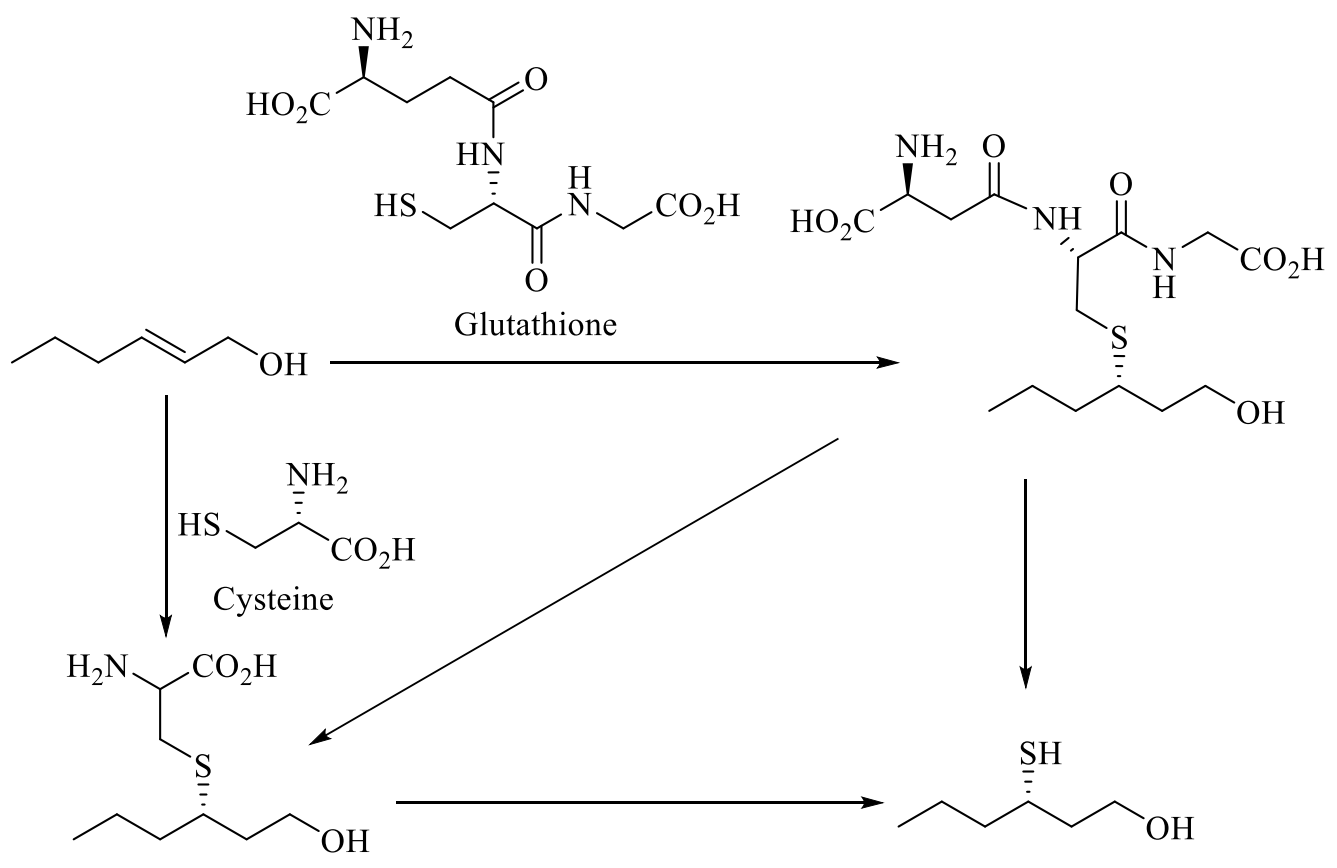

Scheme 2: Possible biosynthesis pathway for thiol synthesis

The biosynthetic pathway that leads to the production of the cysteine-bound conjugates has not yet been completely described, and there are still many discrepancies between the synthesis and final concentration of products, however there is some recent research that is leading in this direction.

\subsubsection{Hexenal/Hexenol Conversion}

It is possible that 2-hexenal, which is plentiful in the grape due to breakdown of linolenic acid via the lipoxygenase pathway following grape damage, is reduced to 2-hexenol and subsequently bound to cysteine to from Cys-3SH. Following this, $3 \mathrm{SH}$ could be cleaved by $\beta$-lyase during yeast fermentation. As 3SHA conjugates of cysteine are also present in the grape, acetylation would occur at some stage during the development to give 
Cys-3SHA. ${ }^{20}$ Subileau et al. have found (with deuterated analogues) that Cys-3SH was converted into $3 \mathrm{SH}$ but they noted that it only accounted for approximately $5 \%$ of the $3 \mathrm{SH}$ present in Sauvignon blanc. Their research with deuterated $E$-2-hexenal as a precursor also indicated that it was not a significant contributor to $3 \mathrm{SH}$ concentrations in wine. ${ }^{20}$ However, recent research by Harsch et al. has found that E-2-hexen-1-ol and $E$-2-hexenal can be interconverted and that E-2-hexenal actually has a higher thiol conversion rate in a model medium than E-2-hexen-1-ol (58.8\% compared to $9.4 \%$ ). When only E-2-hexenal is in the presence of yeast, $20-30 \%$ of it is converted to $E$-2-hexen-1-ol which gives an alcohol/aldehyde ratio of approximately 0.6-0.7 before both compounds were converted by the yeast (probably to hexen-1-ol and hexyl acetate). However, when $E$-2-hexen-1-ol was initially in higher concentrations than $E$-2-hexenal, the yeast favoured a conversion of the alcohol to the aldehyde to give an alcohol/aldehyde ratio between 1.02 and $1.42 .^{21}$

In order to find other thiol precursors, a reliable method of detection must be developed to detect low levels of thiols in wine. The data of Harsch et al. has been interpreted to show that $E$-2-hexen-1-ol acts as a precursor to the volatile thiols. ${ }^{21}$ Initially the yeast converts it to E-2-hexenal that can then go on to react with $\mathrm{H}_{2} \mathrm{~S}$ (or a similar thiol donating entity). This being the case, the initial concentration of the alcohol in the grape juice can have a major effect on the concentrations of $3 \mathrm{SH} / \mathrm{A}$ in the resulting wine. For example, if the amount of $E$-2-hexen-1-ol in the grape juice is greater than $E$-2-hexenal, $3 \mathrm{SH} / \mathrm{A}$ synthesis could be enhanced. However, if initially there is less $E$-2-hexen-1-ol than $E$-2hexenal in grape juice, the yeast may favour the interconversion of the aldehyde to the alcohol and lead to a potential decrease in 3SH/A produced. ${ }^{21}$ This indicates the importance of being able to pinpoint the biosynthetic pathway by which the thiols are formed and in order to do this effectively, a very sensitive technique for analysis of thiols is necessary.

\subsection{Current Thiol Analysis}

GC-MS is a powerful analytical tool for analysing organic compounds. The machine is simple to use and provides significant qualitative and quantitative information. ${ }^{22}$ The GCMS combines a gas chromatograph (which separates the volatile components of a sample), with a mass spectrometer (which helps to identify the individual components of the sample). When a wine sample (Brancott Estate Sauvignon blanc 2011) is extracted using 
liquid-liquid partitioning against dichloromethane, the organic extract can be injected into the GC-MS and the resulting chromatogram can be analysed (Figure 8).

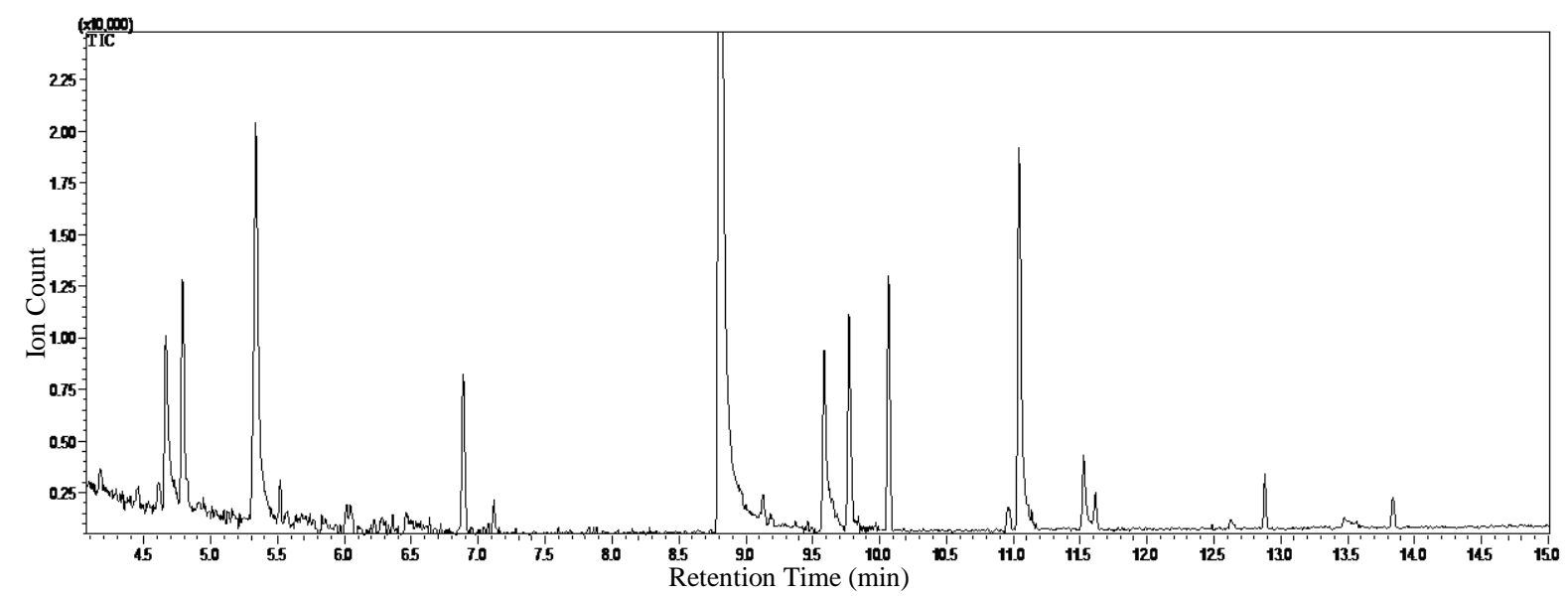

Figure 8: Representative chromatogram of an organic extract (dichloromethane) of Sauvignon blanc.

As can be seen in Table 4, a variety of acids, esters and alcohols can be identified in a Sauvignon blanc sample. However, the thiols that are responsible for this wine's characteristic fruity flavours cannot be detected. This is due to the very low concentrations of thiols present in the wine $\left(<0.01 \mu \mathrm{g} \mathrm{L}^{-1}\right) .^{9}$ This means that a means of concentrating the thiols, or specifically extracting or detecting them is needed, in order to get qualitative and quantitative results.

Table 4: Compounds tentatively identified in an extracted wine sample by GC-MS

\begin{tabular}{cc}
\hline Retention Time & Name* $^{*}$ \\
\hline \hline 4.660 & 1-Hexanol \\
4.790 & 3-Methylbutyl ethanoate \\
5.335 & 4-Hydroxy-butanoic acid \\
6.885 & Ethyl hexanoate \\
8.805 & Phenylethyl alcohol \\
9.585 & Octanoic acid \\
9.770 & Diethyl butanedioate \\
10.065 & Ethyl octanoate \\
\hline
\end{tabular}

$11.045 \quad$ 2-Hydroxy-butanedioic acid diethyl ester

*Compounds tentatively identified by mass spectral fingerprint matching to NIST 11 library.

The current "state of the art" method for thiol analysis uses highly toxic organomercury chemicals ( $p$-hydroxymercuribenzoate - Figure 9) that can be detrimental to human health and the environment, it is not particularly reproducible between labs and it is very time 
consuming - it is for these reasons that many laboratories will not use this methodology. ${ }^{23}$ There are, however, a few methods that have been developed that do not include the organomercury compounds but instead use complicated detectors to analyse the thiols. To this end, it would be beneficial to develop a novel method that is safer, widely available to wineries and laboratories, and more reproducible between labs.

\subsubsection{Organomercury Extraction}

The organomercury extraction method for thiol analysis involves the isolation of an organic phase containing the thiols which can then be extracted with $p$-hydroxymercuribenzoate ( $p \mathrm{HMB}$, Figure 9) where the mercury binds to the sulfur of the thiol. This is followed by a basic anion exchange column in which the benzoate functionality binds to the stationary phase and the other components of the wine are eluted from the column. The column is then eluted with cysteine which displaces the thiol from the mercury and the thiols are eluted from the column so that they can be re-extracted, dried and concentrated. ${ }^{24}$ Once the thiols have been concentrated, they are analysed using a MS. This method of analysis has been widely used in food and wine chemistry to analyse the levels of sulfur containing molecules in foodstuffs.

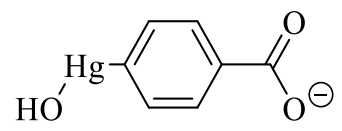

Figure 9: $p$-hydroxymercuribenzoate.

\subsubsection{Development of the Method}

Initially Tominaga et al. developed this method to extract and identify thiols in Sauvignon blanc which led to the first identification of $3 \mathrm{SH}, 4 \mathrm{MSPOH}$ and $3 \mathrm{MSBOH}$ in wines. ${ }^{17}$ Once these compounds were identified they were able to determine where in the wine-making process the compounds arose. It was determined that they were not present in the must, but in fact occurred during the alcoholic fermentation. ${ }^{17}$ Further research, combining this method with a cyclodextrin capillary column for gas chromatography, also allowed researchers to extract enough $3 \mathrm{SH}$ and $3 \mathrm{SHA}$ from wines to establish the enantiomeric ratios in which they were present. $^{25}$

\subsubsection{Detection of Thiols in Cheese}

Not only is this method used in the detection of low concentrations of thiols in wines, but it has also been deemed a selective extraction agent for thiols in cheeses. In 2008 Landaud 
and co-workers used $p \mathrm{HMB}$ to selectively extract thiols from the complex matrices of both Camembert and Munster cheeses for analysis. ${ }^{26}$ Initially researchers pursued whether there were indeed thiols in the cheese that were able to bind to the $p \mathrm{HMB}$. Using sensory analysis in the form of a triangle test, they were able to determine that controls (without $p \mathrm{HMB}$ ) smelt of fruity, sulfur and cheese whereas the samples containing $p \mathrm{HMB}$ were significantly stronger than the controls and the aromas typical of these samples were described as solvent, ammonia, cheese rind, alcohol, and white cheese. These results specified that there were thiols in the samples able to bind to the mercury compound and in doing so the aroma activity of theses thiols was decreased. ${ }^{26}$ Their study further compared three methods of extraction using this organomercury complex and found that the most effective way to extract the thiols from the complex matrix of the cheese was to take small pieces of surface cheese, dissolve them in dichloromethane containing a known amount of hexane thiol as an internal standard and agitate them for 18-20 hours. The samples were then filtered through glass wool and extracted twice using $p \mathrm{HMB}$ in a Tris buffer solution. Following extraction the samples were purified by a basic anion exchange column in which the volatile thiols were released from the mercury by percolation with a cysteine solution. The resulting eluates were sequentially extracted with dichloromethane that was spiked with thiazole (external standard), dried and then injected into a GC either coupled to a Mass Spectrometer or a Pulse Flame Photometric Detector (PFPD) for further analysis and identification. From the results of these studies they were able to identify for the first time, 3-sulfanylpropionate as a powerful aroma compound in Munster and Camembert cheeses. ${ }^{26}$

\subsubsection{Thiol Analysis by Gas Chromatography}

There are currently several methods of analysis that do not include mercury reagents, but they require specialised detectors or complex derivations of the substrate. This is because gas chromatography is highly applicable to the detection of volatile, organic compounds such as those responsible for many flavours and aromas in food and drink. Gas chromatographs can be coupled with a variety of detectors to aid in the elucidation of the unknown analytes in a sample, many of which enhance the sensitivity of the analysis.

\subsubsection{Negative Chemical Ionisation}

In 2006 Ferreira and co-workers proposed a method for thiol analysis using a pentafluorobenzyl bromide $(\mathrm{PFBBr})$ derivatisation and Head Space Solid-Phase Micro-Extraction (HS-SPME) techniques for extraction prior to GC-Negative Chemical 
Ionisation-MS (GC-NCI-MS) injection. ${ }^{27}$ However in 2010, they used a modified version of this method for the analysis of five thiols in wines. ${ }^{28}$ They used a combination of derivatisation of thiols with 1,8-diazabicyclo[5.4.0]undec-7-ene (DBU) and PFBBr in hexane and subsequent analysis with GC-NCI-MS. ${ }^{28}$ In order to prepare the samples for analysis, the wine was spiked with EDTA (Ethylenediaminetetraacetic acid), L-cysteine chlorohydrate and an ethanolic solution of 2-phenylethanethiol (internal standard). Once these were combined, $O$-methylhydroxylamine was added and the sample was purged with nitrogen while heating to $55^{\circ} \mathrm{C}$. The subsequent sample was then loaded onto a solid phase extraction cartridge upon which some of the other major volatile components were removed before the sample was eluted through a solution of DBU and then PFBBr (Figure 10). The derivatised analytes were finally eluted with a solvent mixture of hexane and diethyl ether and then octafluoronapthalene in hexane was added. Following this, the sample was washed with a sodium chloride solution and dried with anhydrous sodium sulphate before injection into the GC-NCI-MS system. ${ }^{28}$

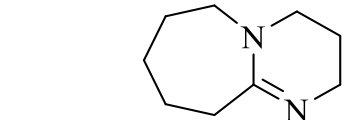

1,8-Diazabicyclo[5.4.0]undec-7-ene

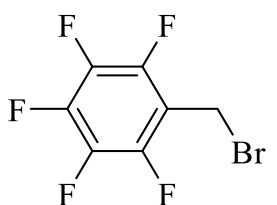

pentafluorobenzyl bromide

Figure 10: Derivatisation agents for thiol analysis.

While this method gave satisfactory determinations of concentrations at very low detection limits, it requires a range of solvents and destroys the natural matrix of the wine, meaning that the analysis takes place outside of the natural conditions that would be present in the grape and throughout the production and consumption processes. Another limitation of this process is that NCI detectors are not always readily available. ${ }^{28}$

\subsubsection{Micro-Extraction/ Stable Isotope Dilution Analysis}

Further methods were described by Capone et al. in 2011 that followed a similar derivatisation method as Ferreira, however, noting that NCI detectors are not readily available, trialled Head Space Solid-Phase Micro-Extraction/Stable Isotope Dilution Analysis GC-MS (HS/SPME/SIDA/GC-MS). ${ }^{29}$ For liquid-liquid extraction, different solvent compositions were evaluated to determine the best solvent for the extraction of $3 \mathrm{SH}$ from white wines, the different solvents included pentane, mixtures of pentane/dichloromethane and pentane/ethyl acetate. These were then extracted with aqueous $\mathrm{NaOH}$ solution. Following this EDTA and $\mathrm{NaCl}$ were added to the samples. In 
order to remove any residual organic solvent, the samples were purged with a stream of nitrogen before a PFBBr solution was added to the sample (Scheme 3). The $\mathrm{pH}$ was then adjusted to between four and five and extraction took place using HS/SPME/SIDA/GC-MS. ${ }^{29}$

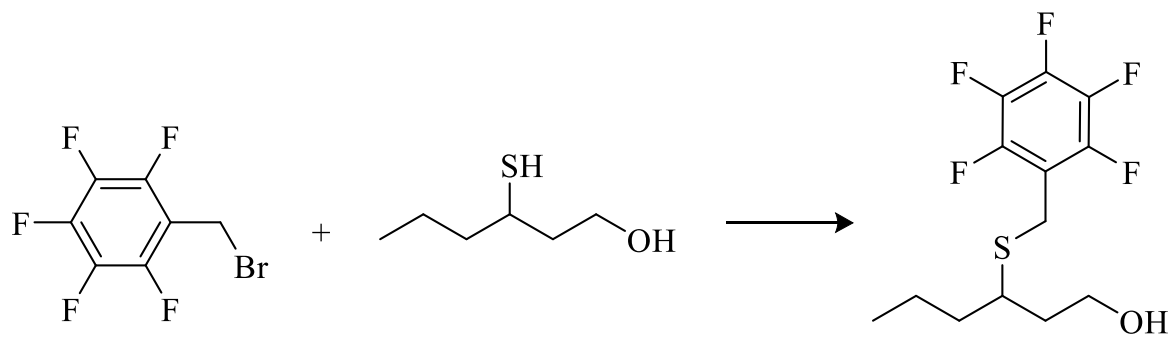

Scheme 3: Derivatisation scheme of the reaction of PFBBr with 3SH.

This method allowed the researchers to investigate the relationship between free thiols and related conjugates in both grape juice and wine. While this method addressed the need for a more available detector than NCI, it did not acknowledge the need for a more representative method of analysis in terms of the wine matrix and consumer conditions.

\subsubsection{In-Needle-Needle Trap Device/In-Fibre-SPME}

In 2012 Warren et al. measured thiol concentrations in garlic using Automated Headspace In-Needle-Needle Trap Device-Gas Chromatography-Mass Spectrometry (In-Needle-NTD-GC-MS) and Derivatised In-Fibre-SPME-GC-MS analysis. ${ }^{30}$ They prepared thiols samples under inert conditions to reduce the stench and diluted the samples with $90 \%$ water and $10 \%$ methanol to $1000 \mathrm{mg} \mathrm{L}^{-1}$. N-Phenylmaleimide was used to derivatise the thiols (Scheme 4 ) and a derivatisation yield of over $\sim 80 \%$ was achieved.<smiles></smiles>

Scheme 4: Derivatisation scheme of the reaction of $\mathbf{N}$-phenylmaleimide with ethanethiol

Using this derivatisation they were able to analyse and assess the thiol compounds present in garlic samples. They found that this method increased the GC peak intensity by over 100 times in comparison to the non-derivatised thiols which allowed great ease of elucidation in terms of attributing peaks to specific thiols and separating them from other components in the matrix. ${ }^{30}$ 


\subsection{Aim}

The aim of the current project was to continue the development of a novel gold based extraction method for analysing volatile thiols. The method combined the well-documented facile formation of a gold-sulfur bond and use of simple analysis techniques like SPME which will hopefully make the method more environmentally friendly, less time consuming and more reproducible between labs than current methods. It has been our aim to develop a product that can encompass all of the above, be accessible to the wine makers and analysts and be easy to use. In light of previous research in this area finding inconsistencies while developing the method (detailed in Chapter 2), it was necessary to take a deeper look many of the components that made up the analytical protocol.

- The kinetics and thermodynamics of the system.

- Observation of the surface chemistry was thought to give a more in depth understanding of the possibility of contaminants on the surface or unforeseen reactions occurring with the surface that could have hindered previous results.

- Alongside these results it was thought that theoretical studies of the thiols and their reactions with the gold would also prove insightful and aid in this investigation.

These insights were thought to enable the development of a technique to 'reset' the fibre to its original condition in between analyses to gain better control and reproducibility. A cost analysis will also be performed in order to determine if the resulting method would be cost effective as well. 


\section{Extracting Thiols from Wine}

\subsection{Introduction}

In order to design a new, non-toxic method for thiol analysis, researchers at Victoria University developed the idea of using gold nanoparticles to selectively bind to the sulfur containing molecules and remove them from the wine. ${ }^{31}$ Using this technique, it was thought that thiols could be selectively extracted from wine samples in concentrations high enough to be analysed on a simple GC-MS. In order to find an alternative to the toxic organomercury method, they investigated the addition of gold nanoparticles to thiol solutions and then analysing the remaining thiol solution to see if an appreciable decrease in thiol concentration (using GC-MS) occurred. At varying initial concentrations of thiols this was not found to make a noticeable difference and a different approach, using SPME, was investigated. ${ }^{31}$

\subsubsection{Solid-Phase Micro-Extraction}

SPME was developed as a technique for sampling that uses less solvent in analysis, improves time efficiency and allows preparation of samples in the field. ${ }^{32}$ SPME analysis uses fibres (usually made of silica) that are coated in a material specific to the analyte of interest. The analyte (or analytes) can be extracted from solutions and adsorb onto the coating of the fibre. Once the extraction is complete, the fibre can be transferred to the GC-MS for analysis. The fibres used in SPME are typically very fragile and therefore are incorporated into a syringe type device to protect them from breaks and contamination (Figure 11). For analysis, the analyte must be desorbed from the fibre (usually by heat) and during this process, the fibre is cleaned and can then be reused. In comparison

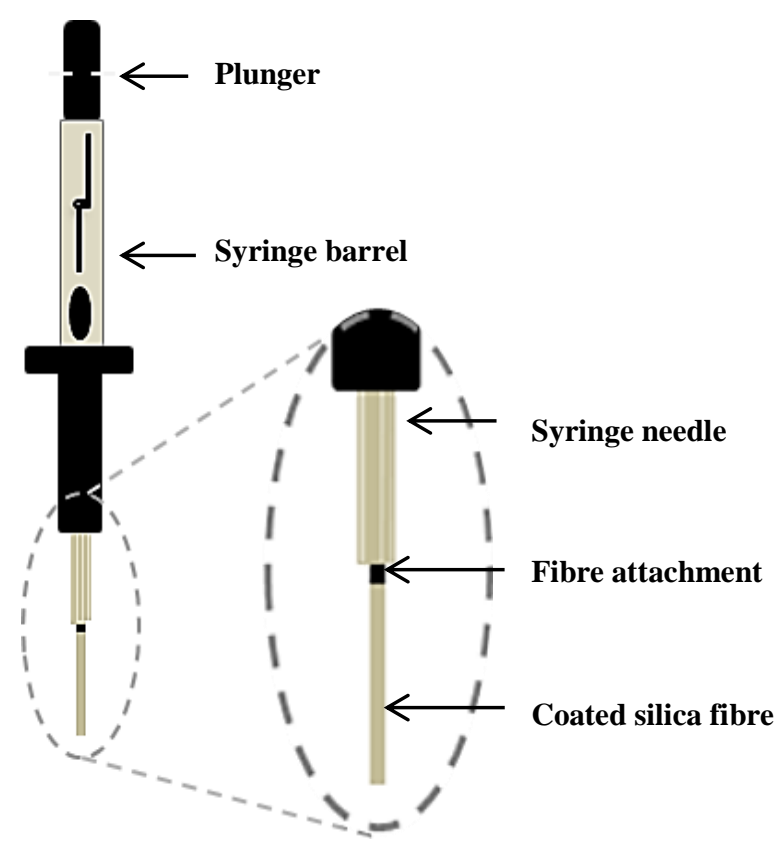

Figure 11: SPME apparatus. to the sample volume, the amount of solvent required for extraction is very small. Another 
benefit of this technique of analysis is that the sample is not exhausted and instead, an equilibrium is established between the sample matrix and the extraction phase and therefore the amount of analyte extracted from the sample is very low. ${ }^{32}$

There are two SPME regimes that are regularly used; direct extraction and head-space extraction. For direct extraction the coated fibre is dipped directly into the sample and the analytes are adsorbed while still dissolved in the sample matrix. However, for head-space extraction the vapour above the bulk solution is analysed rather than the sample medium itself. $^{33}$ This often includes heating of the matrix (and sometimes addition of salt) to help force the volatile analytes into the headspace above the bulk where they can adsorb to the coated fibre (Figure 12). SPME has been widely accepted in the

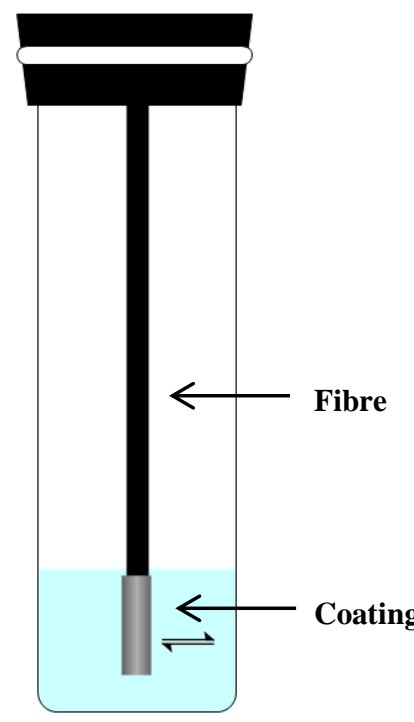

Direct Extraction

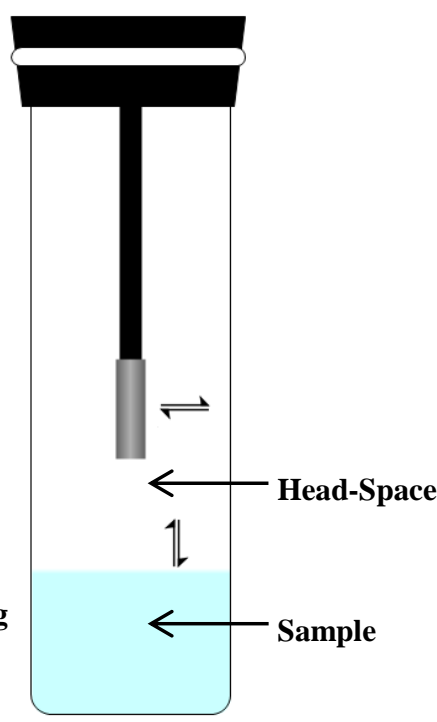

Head-Space Extraction

Figure 12: SPME extraction techniques. food industry, particularly for flavour and aroma analysis and can be successfully coupled with high performance liquid chromatography, gas chromatography and mass spectrometry. ${ }^{32}$ It is also industrially applicable as it can be automated in order to increase time efficiency. ${ }^{34}$ HS-SPME extraction is beneficial to food and beverage analysis as it emulates consumption in that the flavours and aromas that are sensed while eating are typically volatile components of the food or drink that need to be partitioned into the head-space for observation.

When using HS-SPME, several parameters need to be taken into consideration. For example, in 1994 Yang and Peppard demonstrated that the relative liquid and gas volumes of the samples are very important, particularly at low concentrations. The difference between direct extractions for the liquid phase, and head space extractions are the complex equilibria that form (Scheme 5). ${ }^{35}$ In these examples, $A_{1}$ is the analyte present in the liquid phase of the sample and $A_{2}$ is the analyte in the gas phase with $K_{1}$ being the distribution constant between the two phases. Head-space analysis has an additional equilibrium with $\mathrm{K}_{2}$ as the distribution constant between the analyte gas phase and the 
analyte adsorbed to the SPME fibre. While the existence of the gas phase does not necessarily change the distribution between the gas and liquid phases, the volume of the headspace has been seen to influence how much of the analyte will adsorb to the fibre. Yang and Peppard found that in order to obtain highly sensitive results, the sample headspace needed to be kept as small as possible. ${ }^{35}$ With this in mind the current study used $20 \mathrm{~mL}$ sample vials and due to the length of the fibre, used $10 \mathrm{~mL}$ of solution in order to keen the headspace as small as possible while still maintaining a distance between the tip of the fibre and liquid. ${ }^{35}$

a

$\left[\mathrm{A}_{1}\right] \stackrel{\mathrm{K}_{1}}{\rightleftharpoons}\left[\mathrm{A}_{2}\right]$ $\mathrm{b}$

$\left[\mathrm{A}_{1}\right] \stackrel{\mathrm{K}_{1}}{\rightleftharpoons}\left[\mathrm{A}_{2}\right] \stackrel{\mathrm{K}_{2}}{\rightleftharpoons}\left[\mathrm{A}_{3}\right]$

\section{Scheme 5: Generic equilibria expressions for SPME adsorption a) liquid extraction b) gas extraction}

Yang and Peppard also determined that in the majority of trials, as increasing amounts of salt were added to sample, the sensitivity of the SPME sampling changed considerably, thus giving rise to four different types of sample behaviour. The majority of flavour components exhibited increased sensitivity with increased salt concentration (e.g. ethyl butyrate, cis-3-hexenol, benzaldehyde, linalool, $\gamma$-hexalactone, phenylethyl alcohol and cinnamic aldehyde), some samples had an initial increase and then a plateau at higher salt concentrations (cis-3-hexenyl acetate, ethyl acetate, and geranial). The third trend showed that some compounds exhibited an initial increase in sensitivity and subsequently decreases with the addition of more salt (ethyl hexanoate, hexanoic acid,). Finally, some compounds only showed decreasing sensitivity when salt was added to the sample and decreased as more was added (limonene, anethole, and $\beta$-ionone). ${ }^{35}$

As they found that the liquid and headspace extractions differed in kinetics, they investigated the possible differences in rates of evaporation, dissolution, and diffusion in both the gas and liquid phases. ${ }^{35}$ From these analyses they noted that the difference in concentration between the liquid phase and the subsequent gas phase could make SPME liquid and headspace samplings were very different indeed. Typically, the adsorption rate was found to be higher when the concentration of target analyte was increased. It was also noted that if the target analyte was present predominantly in the liquid phase, the liquid extraction was likely to be more effective than headspace analysis (and vice versa) for a given sample volume and time. ${ }^{35}$ 


\subsubsection{Thiols and Gold}

Sulfur containing molecules can bind strongly to coinage metals such as gold, silver, platinum and copper. $^{36}$ The bond strength of a gold-sulfur bond is approximately $210 \mathrm{~kJ} \mathrm{~mol}^{-1}$, which falls within the realms of a typical covalent bond. ${ }^{37}$

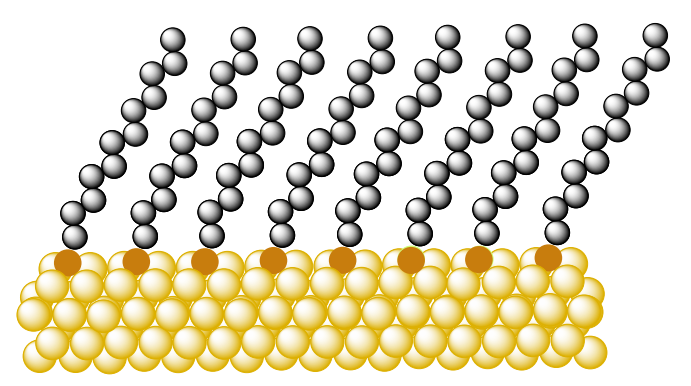

Figure 13: SAM of thiol on gold surface. Gold is advantageous for analytical methodology as it does not oxidise in air below its melting point, and it does not readily react with other chemicals. As a result it can be used under typical laboratory conditions without the need for Schlenk-type equipment (e.g. vacuums and inert atmospheres). Although gold is typically thought of as inert, on the nanoscale it has vast ligand chemistry and can be found in oxidation states ranging from $-\mathrm{I}$ to $+\mathrm{V}$, the most common oxidation states are $+\mathrm{I}$ and + III. Gold surfaces provide a convenient surface for Self-Assembled Monolayer (SAM) formation because they are typically easy to prepare and clean due to the inert properties of gold under ambient conditions. ${ }^{38}$ Long-chain, linear alkanethiols, $\mathrm{HS}\left(\mathrm{CH}_{2}\right)_{\mathrm{n}} \mathrm{X},(\mathrm{X}=$ a terminal functional group $)$ can adsorb onto gold surfaces from thiol solutions to form self-assembled monolayers (Figure 13). ${ }^{39}$ These monolayers form at an angle to the gold surface depending on the facet to which they bind; the $\alpha$-angle has been measured as $28^{\circ}$ for $\mathrm{Au}(111)$ and $14^{\circ}$ for $\mathrm{Au}(100) .{ }^{37}$ The $\alpha$-angle relates to the angle at which the linear thiol rotates away from $90^{\circ}$, meaning that it is not directly perpendicular to the surface in the monolayer. The thiols can also rotate in terms of the $\beta$-angle which will give the relative positioning of the hydrogen atoms along the carbon chain. For $\mathrm{Au}(111)$ this was measured to be $53^{\circ}$ and $70^{\circ}$ for $\mathrm{Au}(100) .^{37}$

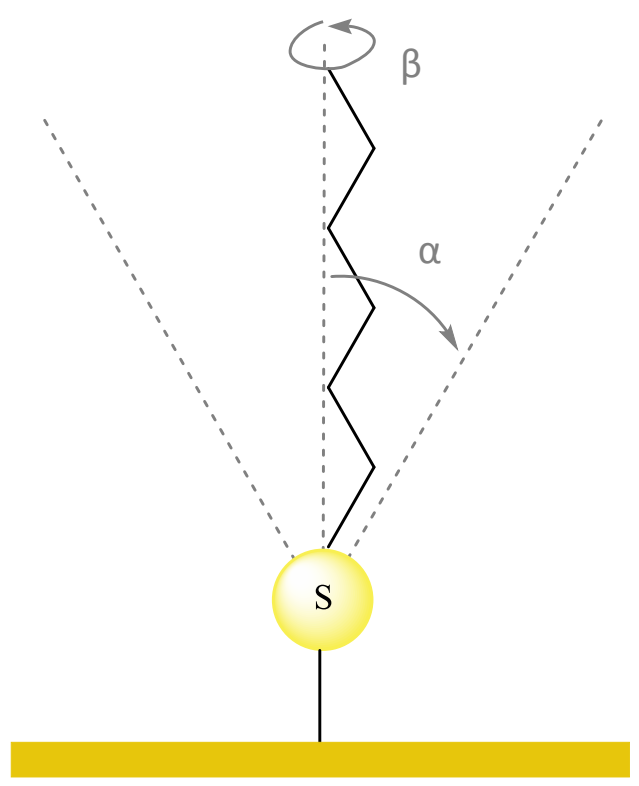

Figure 14: $\alpha$-angle when a linear thiol binds to metal surface. 


\subsubsection{Kinetic and Thermodynamic Factors}

The formation of a linear thiol SAM on a gold surface can be thought of as proceeding via a two-step mechanism. The first step is the initial absorption of the thiol to the surface, this is followed by a reorganisation of these thiols on the surface, which is the rate determining step of this mechanism. ${ }^{40}$ However, in 2010, Low noted that non-linear thiols such as 3SHA, do not form a highly ordered monolayer and that their ability to reorder is limited. The molecules adsorb to the surface by "lying down" parallel to the surface and therefore do not form a densely packed structure, meaning that adsorption is likely to occur almost instantaneously. ${ }^{40}$

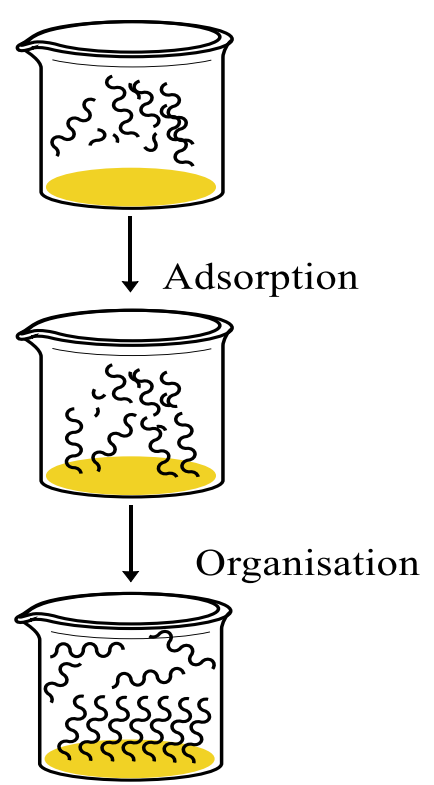

Figure 15: Mechanism of SAM formation

\subsubsection{Non-linear Thiols on Gold}

While it can be seen that long chain linear thiols arrange in reasonably uniform monolayers, non-linear thiols have the ability to form more complex layers on the surface due to increased sterics or functionalities that may change the nature of the packing. The difference

Table 5: Contact angle measurements. Adapted from Low 2010. ${ }^{40}$

\begin{tabular}{cc}
\hline Sample & Contact Angle $\left(^{\circ}\right)$ \\
\hline \hline Au (Clean) & $85 \pm 2.8$ \\
3SH & $80 \pm 1.4$ \\
3SHA & $37 \pm 1.4$ \\
Hexadecane thiol & $113 \pm 0.3$ \\
\hline
\end{tabular}
between the packing of linear and non-linear thiols can be observed in the wetting of the surface as described by Low in $2010 .{ }^{40}$ Glass microscope slides were sputtered with gold and then immersed in thiol solutions, these slides then had a bead of liquid dripped onto them and the contact angles were measured and compared with other thiols. It was found that hexadecane thiol and 3SHA afforded the most significant changes in comparison to the contact angle measure on the clean gold surface (Table 5) and that there was 'reasonable coverage' of the surface with thiol. ${ }^{40}$ This research also looked at the orientation of the thiols in relation to the gold surface and using Polarisation Modulation Infra-Red Reflection Adsorption Spectroscopy (PMIRRAS) found that 3SH and 3SHA lie flat on the gold surface (Figure 16). ${ }^{40}$ 


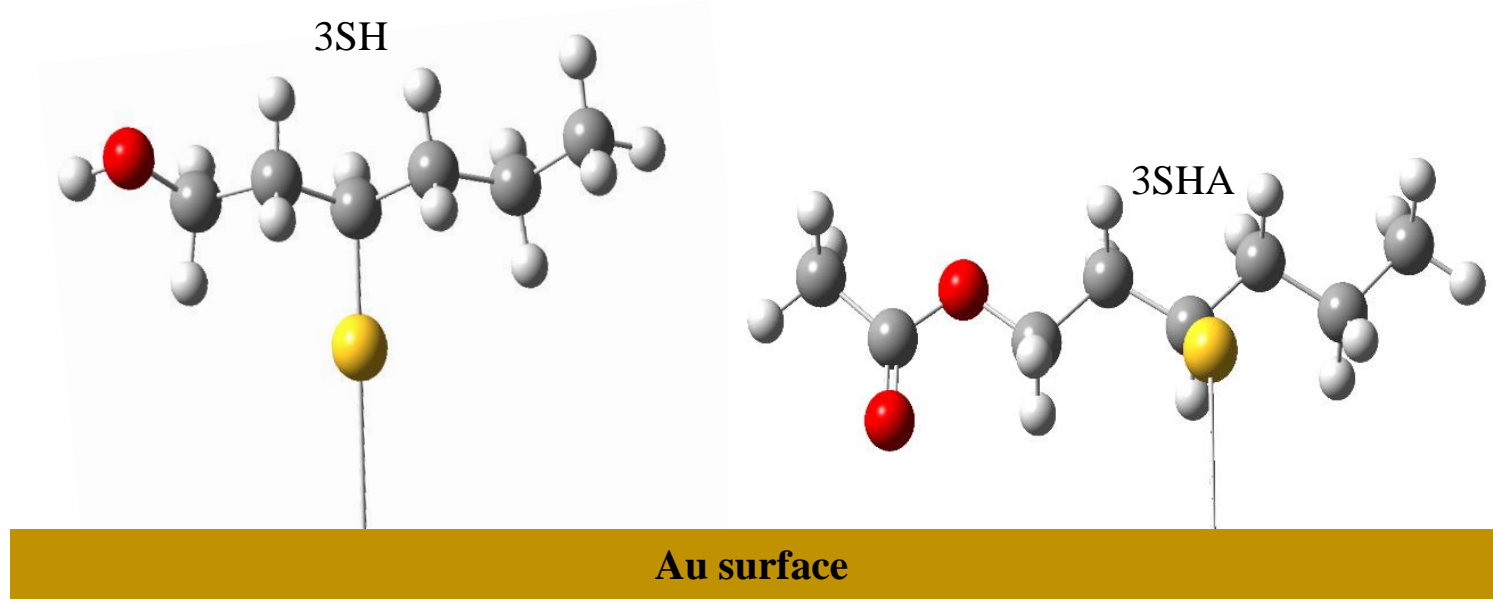

Figure 16: Side View of the orientation of 3SH and 3SHA binding to a gold surface. Adapted from Low 2010. ${ }^{40}$

\subsubsection{Gold Coated Fibre for Thiol Extraction}

Preliminary research at Victoria University followed a procedure in which the polymer coating from an SPME fibre was removed and the core was then coated with gold nanoparticles, this was then used to selectively extract the thiols and successfully detect them in Sauvignon blanc wines. ${ }^{31}$ Further developments of this work included the purchase of uncoated SPME fibres and subsequent silanisation of the fibre prior to gold deposition. ${ }^{41}$ The fibres were immersed in a gold nanoparticle colloid for an hour causing them to turn blue, followed by conditioning at $270^{\circ} \mathrm{C}$ for 1 hour (Figure 17).

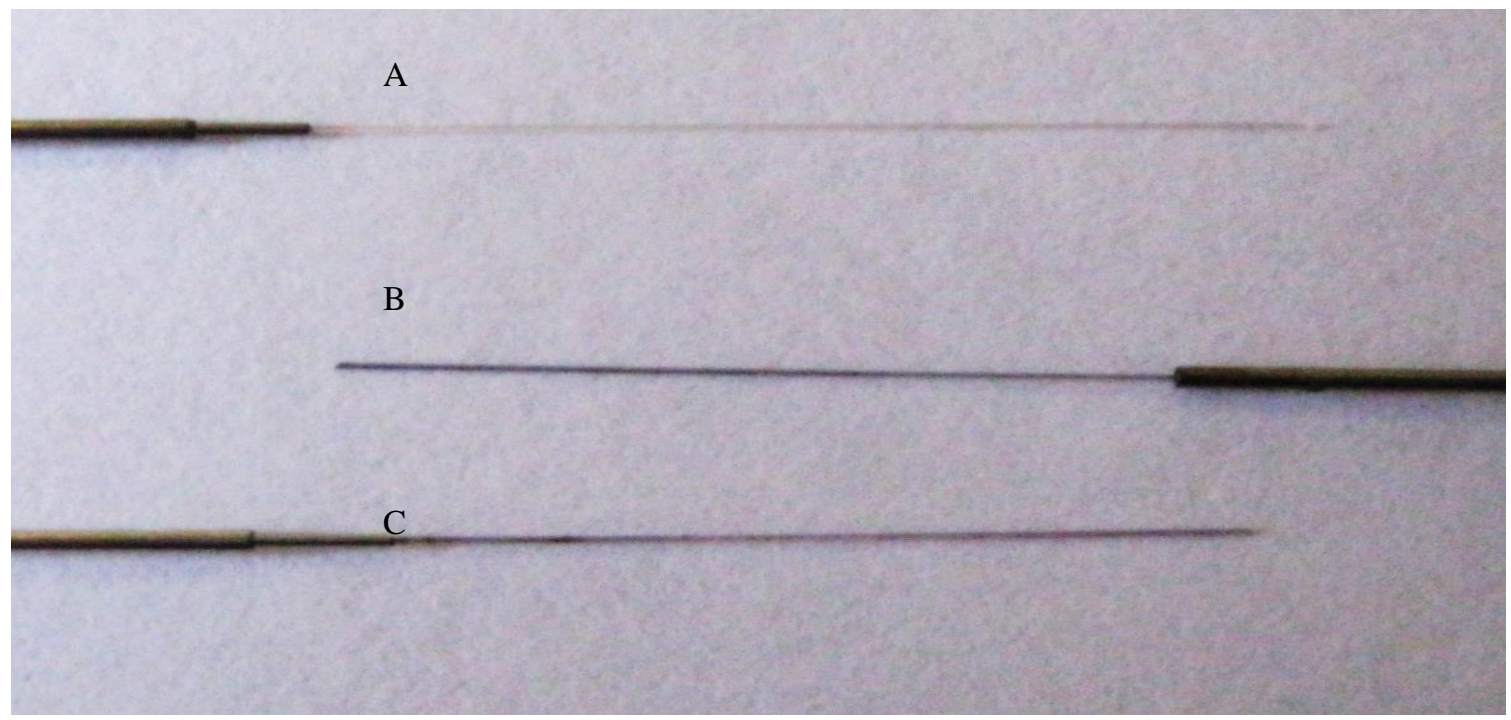

Figure 17: (a) Uncoated, (b) gold coated and coated (c) conditioned. All $2 \mathrm{~cm}$ fibres. ${ }^{41}$

Using this method, thiols were heated in SPME vials to $40^{\circ} \mathrm{C}$ for an incubation period of 15 minutes (to allow the volatile thiols to form an equilibrium between the liquid and the headspace). The fibre was then exposed to the headspace of the sample for an extraction 
period of 30 minutes (to allow the thiols sufficient time to replace the Trisodium Citrate (TSC) on the surface of the gold nanoparticles). Following which the thiols were desorbed from the fibre in the GC-MS (at $270^{\circ} \mathrm{C}$ ) to analyse relative concentrations. While these fibres were able to selectively extract thiols from model wine solutions $\left(1.1 \mu \mathrm{g} \mathrm{mL}^{-1} 3 \mathrm{SH}\right)$ at concentrations far below the detection limits of a standard coated polymer fibre, it was found that over consecutive runs there was a sequential decrease in peak intensity and a need for a significant fibre recovery time between analyses, often longer than a day. It was thought that the problem may have been related to a hysteresis effect, with incomplete desorption of thiols from the fibre surface and/or possible contamination of the fibre surface during or post-injection. ${ }^{41}$ Attempts to overcome this hysteresis, such as varied inlet temperatures, conditioning the fibre in between runs and nitric acid cleaning were pursued. Unfortunately, the latter appeared to damage the fibre and overall there was no change in the inconsistencies observed ${ }^{41}$ and considering fibres are approximately $\$ 300$ each, it is not feasible to buy a new fibre for each sample.

Coincidentally, a recent research project from the University of Auckland was found to have also focused on the investigation of the benefits and limitations of using of gold plated needles to detect 3SH and 3SHA in Sauvignon blanc. ${ }^{42}$ The outside shaft of an SPME needle was coated with metallic gold in order to selectively extract thiols from solution. Direct injection of pure thiol samples was used to demonstrate that detection of thiols could occur using GC-MS and that thiols were detected. However, they had relatively poor detection limits and a noisy baseline, which was due to the non-specificity of direct injection. It was also found that when using gold electroplated needle shafts, the analyses suffered a loss in sensitivity. Over time there appeared to be a reduction in gold affinity for 3SHA, and overall thiol concentration was not always related to peak area on the GC chromatograph. It was thought that the rubber septum of the GC injection port was degrading the gold surface by scraping off coated gold thus accounting for the gold-thiol affinity decrease over subsequent runs, ${ }^{42}$ although coupled with the results from Victoria University of Wellington, it is likely to be a combination of degradation and the aforementioned hysteresis effect.

The research discussed in this thesis focused on the reactions occurring at the interface between the gold and thiols to determine possible causes of the hysteresis. In 2004 Bellino et al. monitored the decrease of the plasmon band intensity, and were able to describe some of the kinetic parameters pertaining to the adsorption of charged thiols to gold nanoparticles with a diameter of $20 \mathrm{~nm} .{ }^{43}$ They noted that as they added increasing 
amounts of thiol to the nanoparticle solution, the radii of the nanoparticles remained the same as the surface coverage increased, and the colour of the colloids shifted from red to blue as they began to aggregate. ${ }^{43}$ Aggregation, however, was not thought to occur during the current studies as the nanoparticles were fused to a silica core, and were therefore unlikely to aggregate due to a change in surrounding molecules. The relative absorbance of the gold nanoparticles decreased over time as 3-mercapto-1-propane sulfonate (MPS) or 2-mercaptoethlyamine hydrochloride (MEA) bound to the surface and it was noted that this process took less than 10 minutes, particularly in the case of 3-mercapto-1-propane sulfonate in which it absorbance is negligible in under 2 minutes. ${ }^{43}$ For the purposes of the current research, this was important to note in terms of establishing the length of time for which the fibre was exposed to the thiols.

\subsubsection{Method of Analysis}

The timing of the analyses is important in obtaining reproducible results. For the current study, a 'dovetailing' method (Figure 18) was employed as the most efficient use of time and allowed for the least amount of potential contamination due to air exposure.

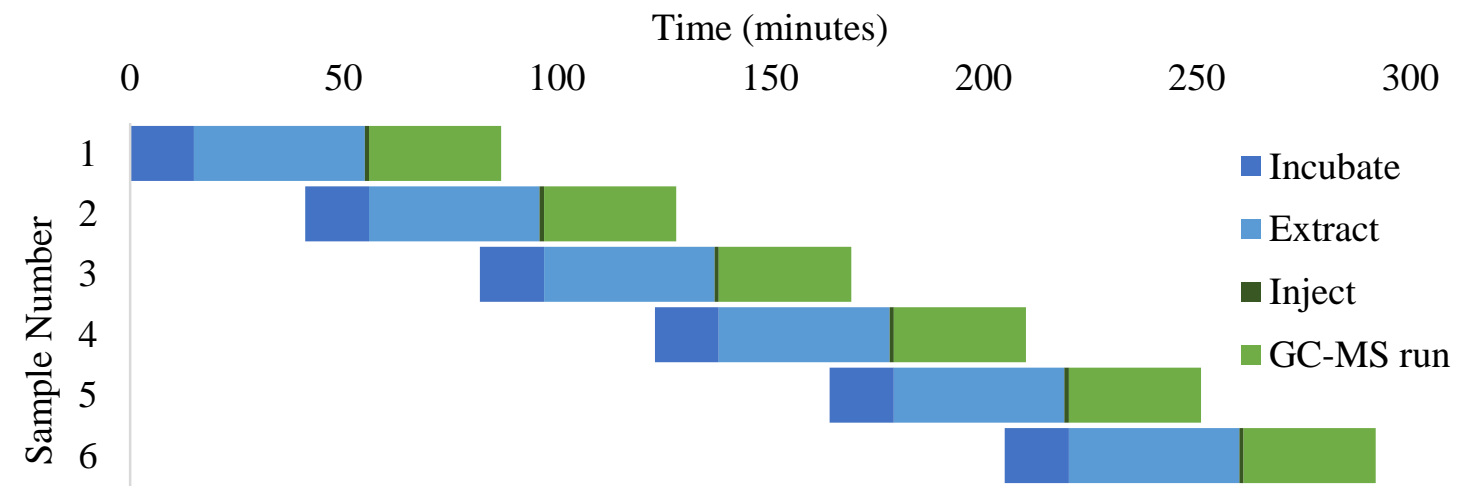

Figure 18: Example of generic SPME sample preparation and GC-MS analysis.

Using this procedure, it was possible to save approximately 3 hours over 6 samples while still maintaining appropriate incubation and extraction periods, when compared to a non-dovetailed, linear protocol. This also allowed for the fibre to be transferred from the sample into the GC inlet port and then directly into a new sample, reducing the fibres exposure to mere seconds as opposed to the potential for there to be 1.5 hours of possible contamination. 


\subsection{Initial Results and Discussion}

\subsubsection{Protocol Standardisation}

The retention time (RT) of each standard was established by comparing the compounds' mass spectral fragmentation pattern to the National Institute of Standards and Technology library $^{44}$ (Figure 19). In some cases, the MS was run in scan mode which monitors a wide range of mass fragments (usually from m/z 50 to m/z 400). However, the majority of samples were run in Selected Ion Monitoring (SIM) mode, where specific ions were selected and only those specific fragments were detected by the MS. SIM mode has lower detection limits than scan as only a small number of fragments are monitored which allowed for more signal to be acquired per second for each selected m/z. As seen in Figure 19 , there are some key fragment ions in the $3 \mathrm{SH}$ MS spectra which was obtained from the National Institute of Standards and Technology library. In subsequent analyses these key fragment ions (m/z values 55, 67, 82, 88, 100, 116, 134) were monitored in SIM mode.

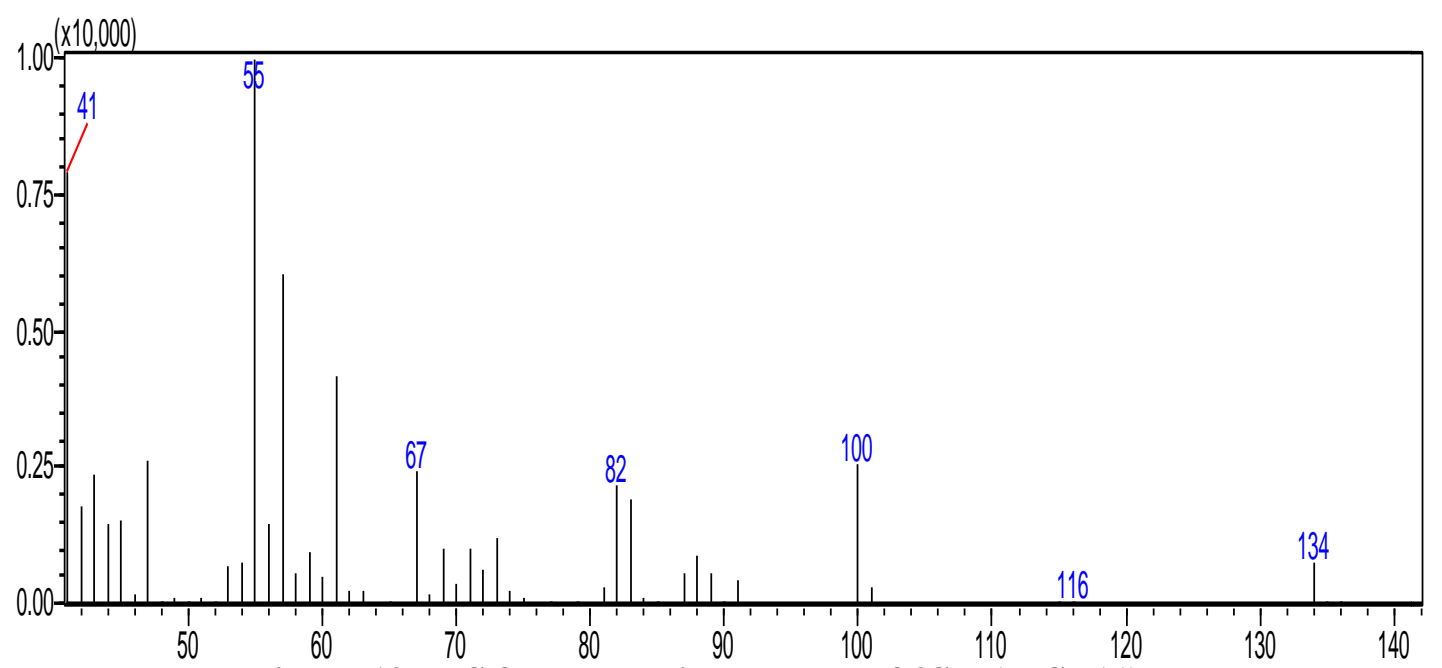

Figure 19: MS fragmentation pattern of 3SH (NIST11).

Typically there is one major fragment ion that is used for quantification (the "quant" ion) and the ratio between it and other fragment ions is used for identification (so-called "qual" or qualifier ions). For example, in the case of $3 \mathrm{SH} \mathrm{m} / \mathrm{z} 55$ should be twice as intense as $\mathrm{m} / \mathrm{z} 67$ and m/z 100, it should also be three times as high as m/z 82, and four times as high as $\mathrm{m} / \mathrm{z} 134$.

In order to test the feasibility for head space analysis, solutions of $3 \mathrm{SH}$ were prepared at a concentration of $60 \mu \mathrm{g} \mathrm{L}^{-1}$ (in water) and $10 \mathrm{~mL}$ of each sample was transferred to an SPME vial with $1 \mathrm{~g}$ salt $(\mathrm{NaCl})$ and stirred during incubation and extraction (15 and 40 minutes respectively). Using the fibre the target analytes were transferred to the injection port and after desorption the fibre was reused in the extraction of a subsequent 
sample. As can be seen in Figure 20, at 8.9 minutes there is a peak indicative of 3SH and three other related impurities, as the quoted 3\% impurity of the 3SH standard solution accounts for. All peaks, over subsequent injections, decrease and fluctuate in size which confirms the hysteresis effect noted in previous research. ${ }^{41}$

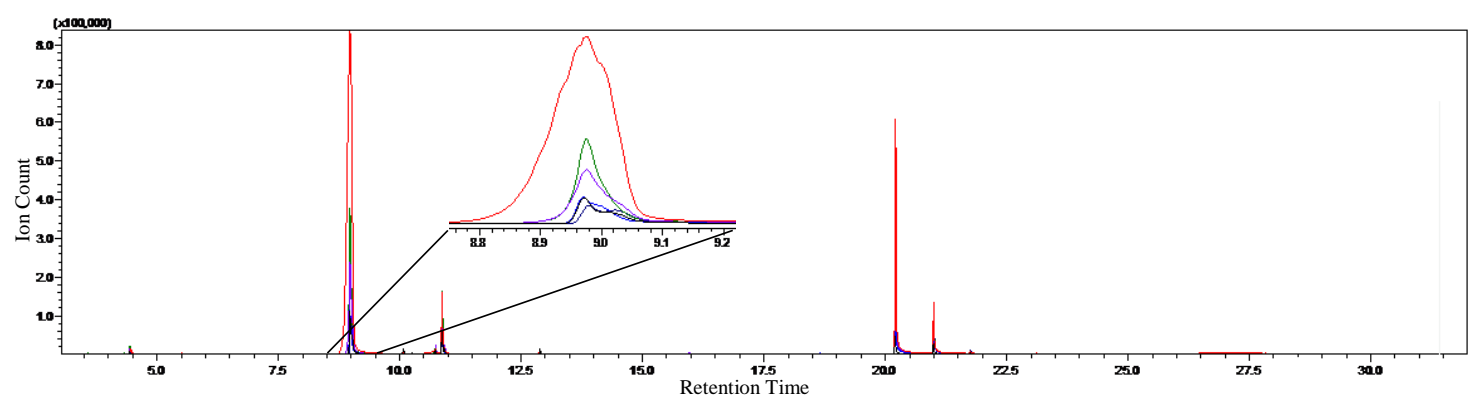

Figure 20: Overlay of HS Chromatograms of the model solution.

In order to rule out contamination from as many sources as possible, the samples were simplified to a bare minimum. Dilute thiol solutions were prepared at a concentration of $60 \mu \mathrm{g} \mathrm{L}-1$ and $10 \mathrm{~mL}$ of each sample was transferred to an SPME vial with MQ triple distilled water and using the same methods as the previous experiment. From the results in Figure 21 it can be seen that the peak area is initially stable within $1 \%$ of the initial run, however, following this there is an increase in peak area of over $1000 \%$ and then a subsequent decrease of $84 \%$. This indicated that a contamination was not the cause of

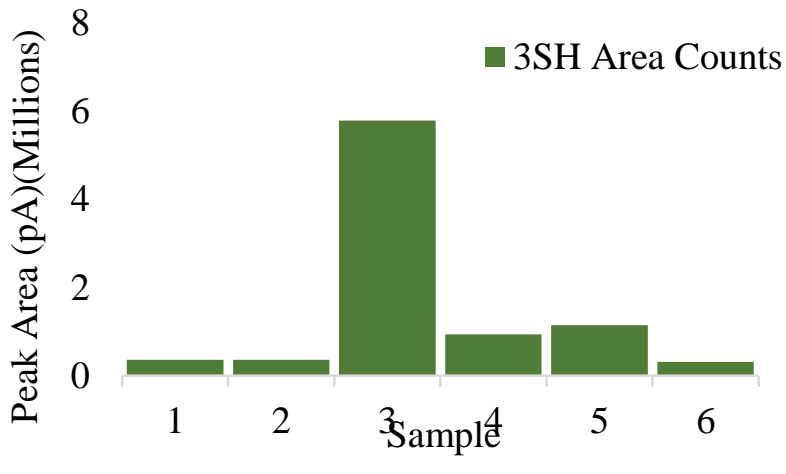

Figure 21: 3SH peak area in model solution over subsequent runs. the inconsistencies seen in the chromatograms of previous research; rather it was likely to be related to inconsistent extraction and desorption of the thiols themselves.

In order to test if any residual thiols were bound to the surface of the gold coated fibre (which may have been a cause for irregular results) was conducted. This analysis included an initial analysis of the standard 3SH sample and then a second injection with the same fibre. If a significant amount of thiol was still present on the fibre, this would have shown in a second peak on the chromatogram, however, this was not the case. This indicates that the temperature of the injection inlet is high enough to fully desorb thiols from the gold coated fibre. 


\subsubsection{Changing time between runs}

It was noted in previous research that there may have been a 'recovery time' for the fibres in order to get reproducible results. An attempt to find the minimum 'relaxation' time was investigated where the fibres were given an increasing length of time to 'recover' in between sets of sample analyses. A comparison can be seen in cluster one (Figure 22) that shows the changes in peak area over subsequent samples of the same concentration on the same day. This fluctuation initially looked as though it would plateau out over time with more samples degrading or interfering with the fibre,

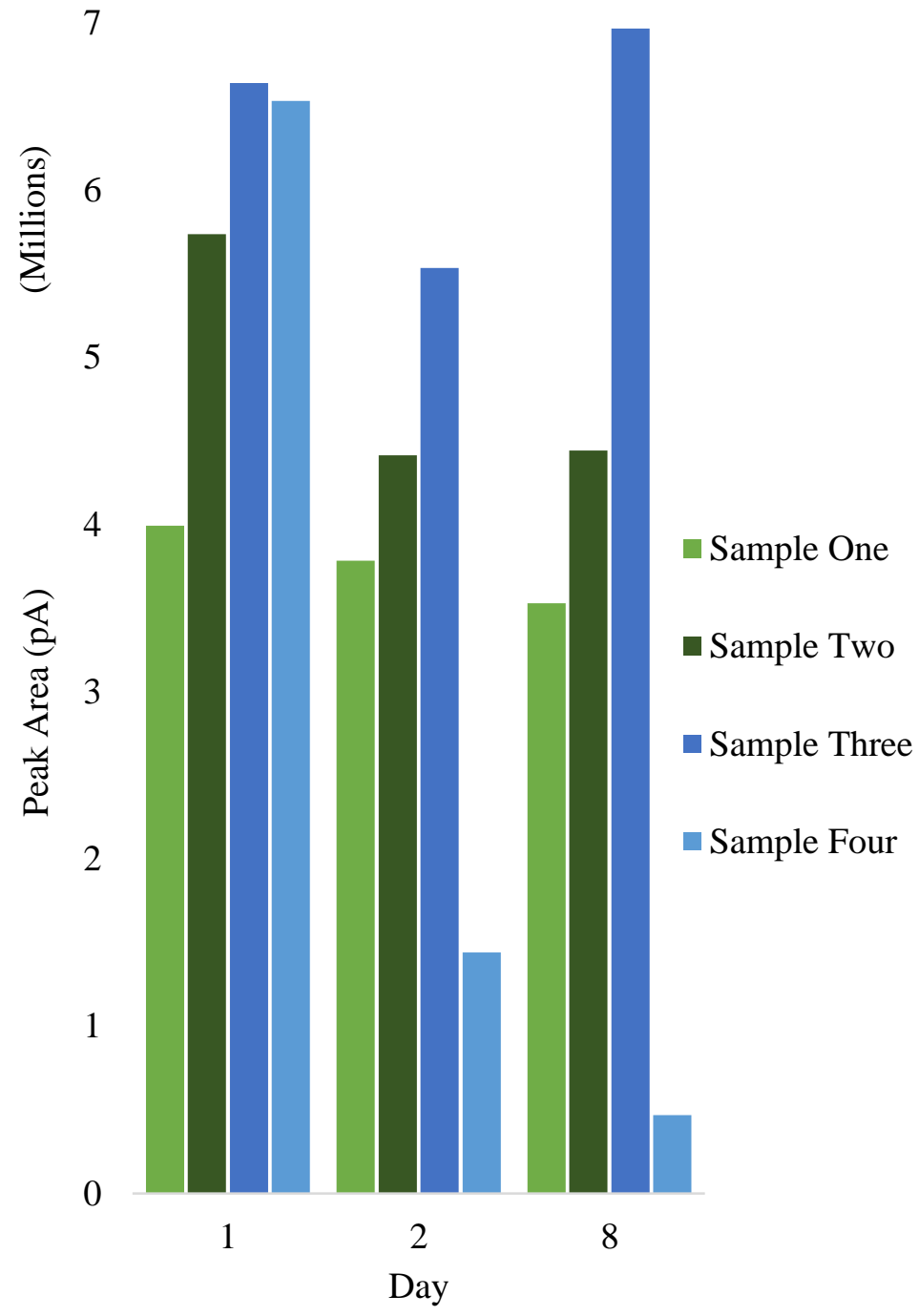

Figure 22: Peak area of 3SH after 'resting' fibre for increasing periods of time.

however, this was not the case and the peak areas continued to fluctuate. While throughout the experiment it was seen that the first three injections exhibited an increase in peak area, the fourth injections displayed decreases, particularly on the second and eighth days. The initial injections on each day were reasonably close in peak area (within $15 \%)$ to one another. Interestingly, on day eight the peak area of sample two was within $1 \%$ of the peak area of the second sample of day two, however, the rest of the injections did not indicate similar trends. As this experiment continued, fibres were 'rested' for increasing periods of time and this was not found to relieve the inconsistencies. 
It was noticed throughout all the results from the initial experiments that there was no predictability to the peak areas observed. Subsequent runs on a day saw the peak areas fluctuate and sometimes deform from narrow peaks to wider peaks with varying degrees of shouldering. This indicated that the desorption of the fibre, or the way in which the thiol interacted with the stationary phase in the GC column, had

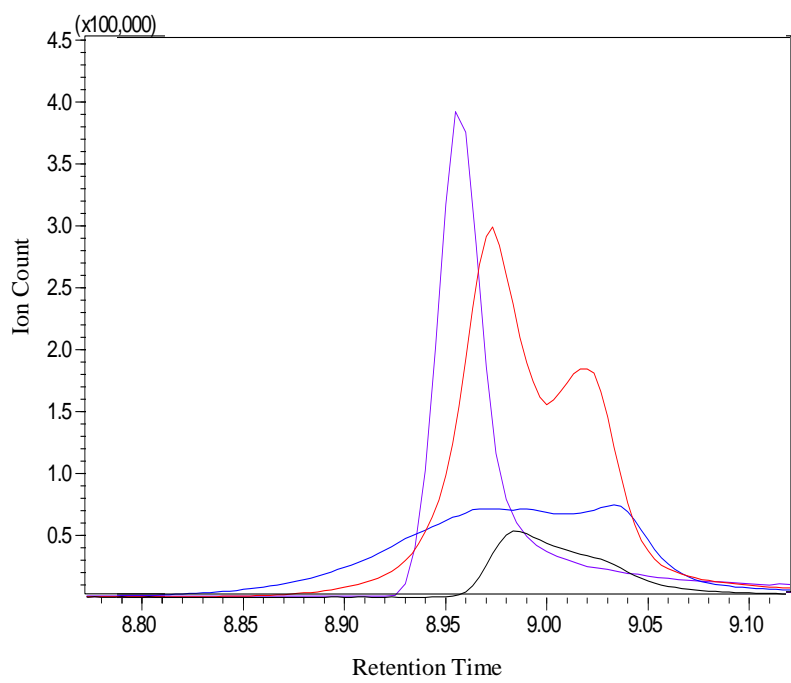

Figure 23: Overlay of $3 \mathrm{SH}$ peak heights of subsequent injections.

1:purple, 2:red, 3:black \& 4:blue

changed. Figure 23 depicts the deformation of the peaks, where the purple trace is the initial run and blue, black and purple represent traces from subsequent traces on the same day, using the same fibre.

\subsubsection{Experimental Time Changes}

Prior to injection into the GC-MS the incubation time and the extraction times of the samples were monitored and changed in order to see if this had an effect on the amount of analyte being adsorbed to the fibre and then detected in the GC-MS. As can be seen in Figure 24 the incubation and extraction times were not reliably correlated to the $3 \mathrm{SH}$ peak area observed on the GC chromatogram. This suggests that regardless of the time, reproducible adsorption and desorption at the surface was not being achieved.

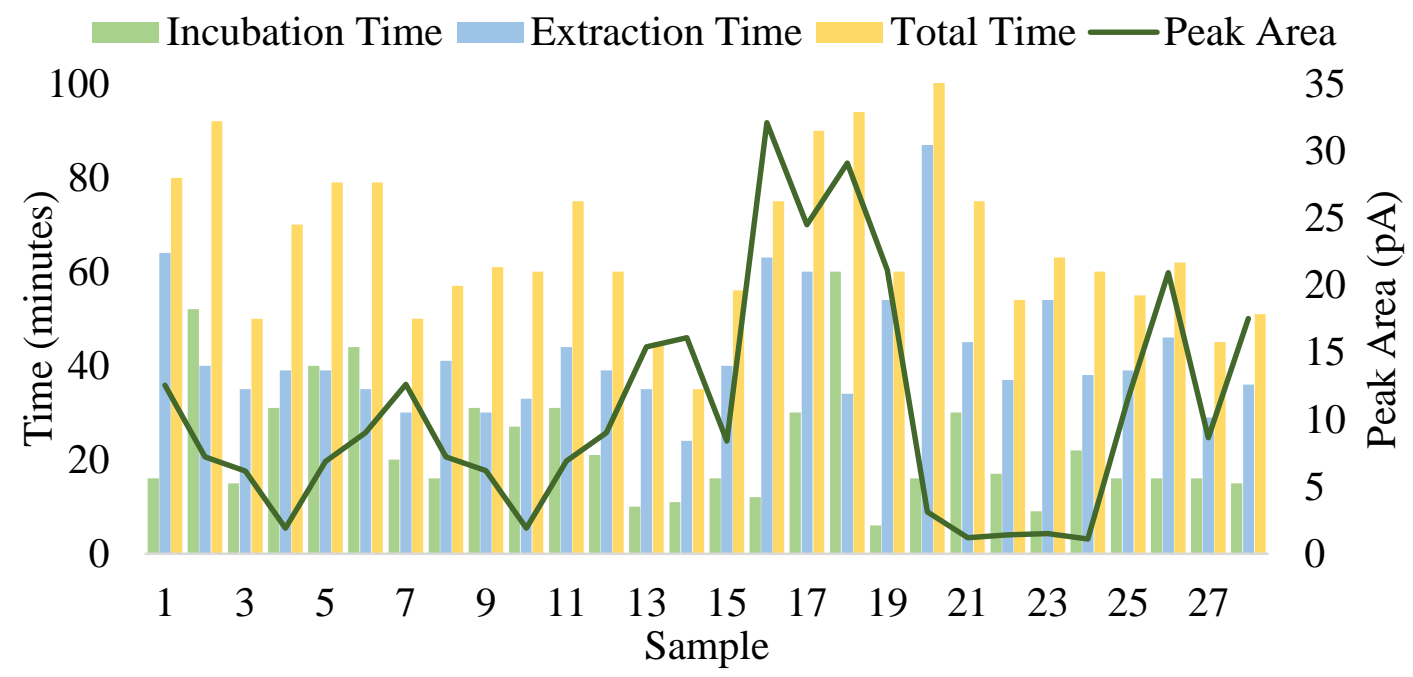

Figure 24: Experimental timings during protocol in relation to the 3SH peak area observed. 


\subsubsection{Salt addition}

In order to test the ability for head space analysis solutions of $3 \mathrm{SH}$ were prepared at a concentration of $60 \mu \mathrm{g} \mathrm{L}^{-1}$ and $10 \mathrm{~mL}$ of each sample was transferred to an SPME vial with $1 \mathrm{~g}$ salt but not stirred during incubation and extraction (15 and 40 minutes respectively) before being injected into the GC-MS. As with previous experiments it can be seen that the peak area of the $3 \mathrm{SH}$ varies with each injection and is not predictable.

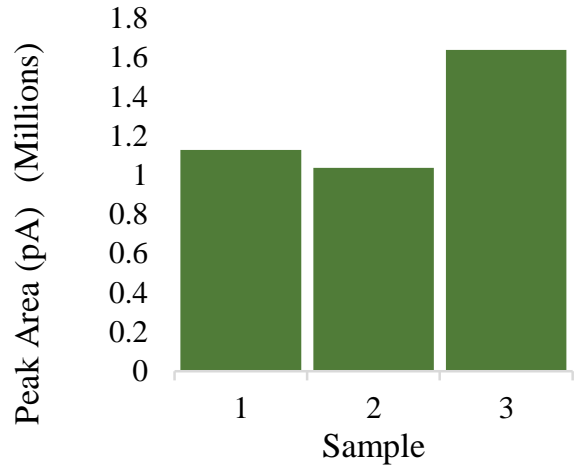

Figure 25: 3SH Peak areas of subsequent injections ( $\mathrm{NaCl}$ in sample matrix)

\subsubsection{Direct Extraction}

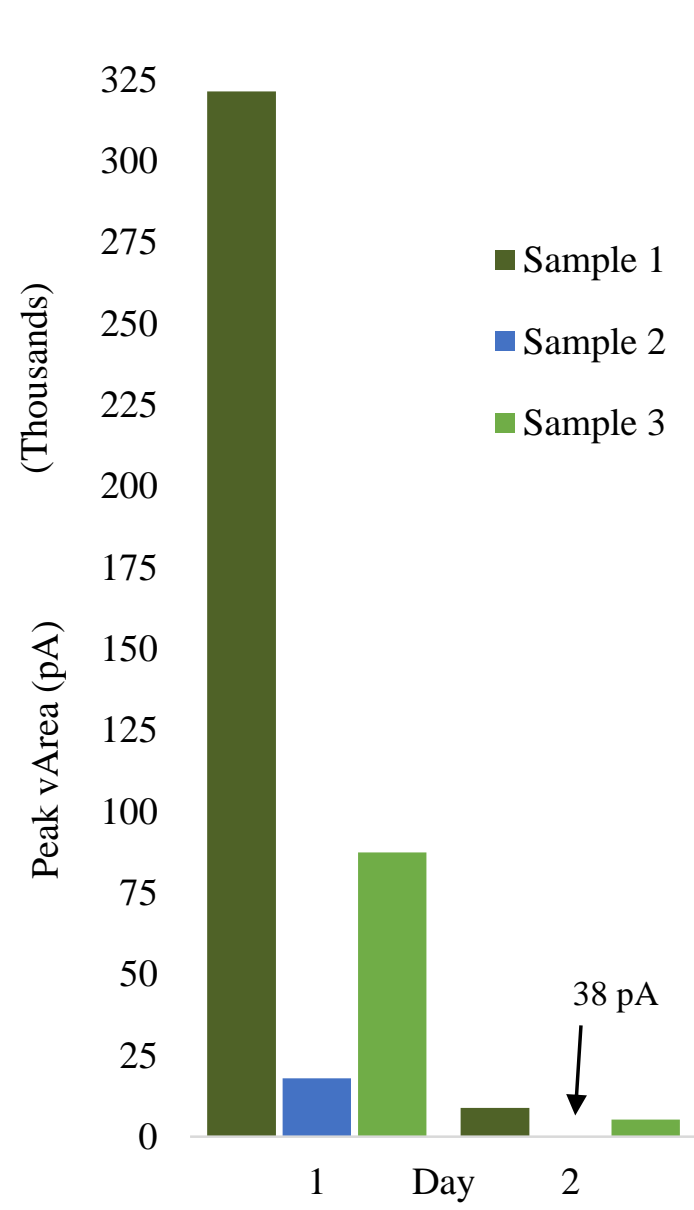

Figure 26: 3SH Peak area of liquid extractions.

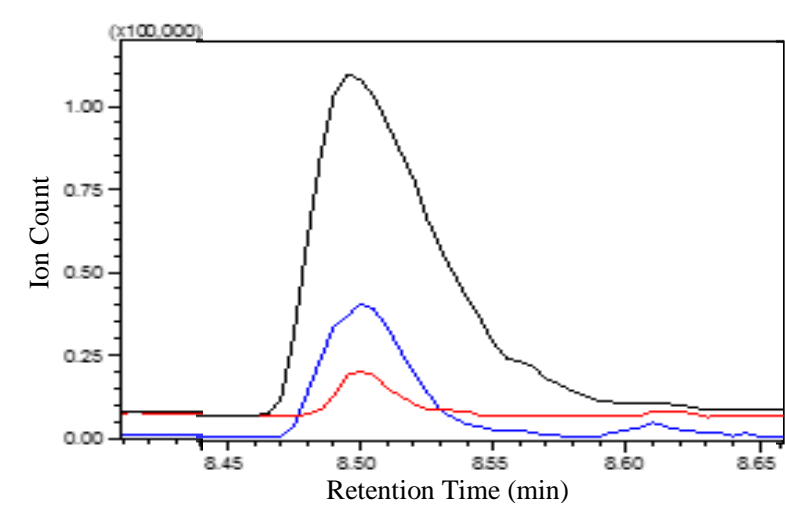

Figure 27: 3SH peak due to a direct dip. 1:black, 2:red \& 3:blue

Instead of using headspace analysis it was proposed that direct extraction into the liquid would provide a more reproducible environment for adsorption and that this might aid in the kinetics of the binding to the surface. $15 \mathrm{~mL}$ of $60 \mu \mathrm{g} \mathrm{L}^{-1} 3 \mathrm{SH}$ was transferred to an SPME vial and was directly extracted for 30 minutes using the gold coated SPME fibre. In Figure 26 it can be seen that this did not change the variability of the peak area over subsequent samples and days. It can also be seen in Figure 27 that the peak shape changes on subsequent runs as well as the fluctuation in peak area that occurred. 


\section{Characterisation of the Surface and Binding}

\subsection{Introduction}

To properly analyse the surface of the gold nanoparticles and their interaction with the thiols, it was important to develop a more in-depth understanding of the properties of the individual constituents of the gold surface and thiol ligand, and then examine combinations of their properties, both experimentally and theoretically. Examination of the surface of the gold nanoparticles was thought to give an insight to the chemistry that was occurring and possible sources of contamination or degradation at the thiol-gold interface. Initially, microscopy techniques were employed in order to characterise the size and shape of the nanoparticles, this was able to give initial parameters by which appropriate Surface-Enhanced Raman Scattering (SERS) spectra could be obtained and analysed. In combination with computational chemistry, it was possible to develop a theoretical picture of the chemistry occurring at the surface of the fibre during thiol binding and desorption.

\subsubsection{Physical Characterisation}

The physical properties of a nanoparticle can influence the way it looks and how it behaves. By comparing the physical characteristics of the synthesised nanoparticles with data collected from previous research, it was possible to identify the size and stability of the nanoparticles, which could give an indication of their chemistry and a starting point from which to extrapolate possible reactions and therefore, contaminations or degradations.

In 2007, Njoki et al. investigated the correlations between the optical properties of gold nanoparticles and their size. ${ }^{45}$ They synthesised nanoparticles of sizes ranging between 10 and $100 \mathrm{~nm}$ and analysed them using both UV-Vis and SERS. Due to the surface plasmon resonance effect attributed to gold nanoparticles, the observed colour of their colloids is related to their size. It was found that as the size of the particle increased from 30 to $90 \mathrm{~nm}$, the colour of the colloid transitioned from dark red to pink (Figure 28). ${ }^{45}$ 


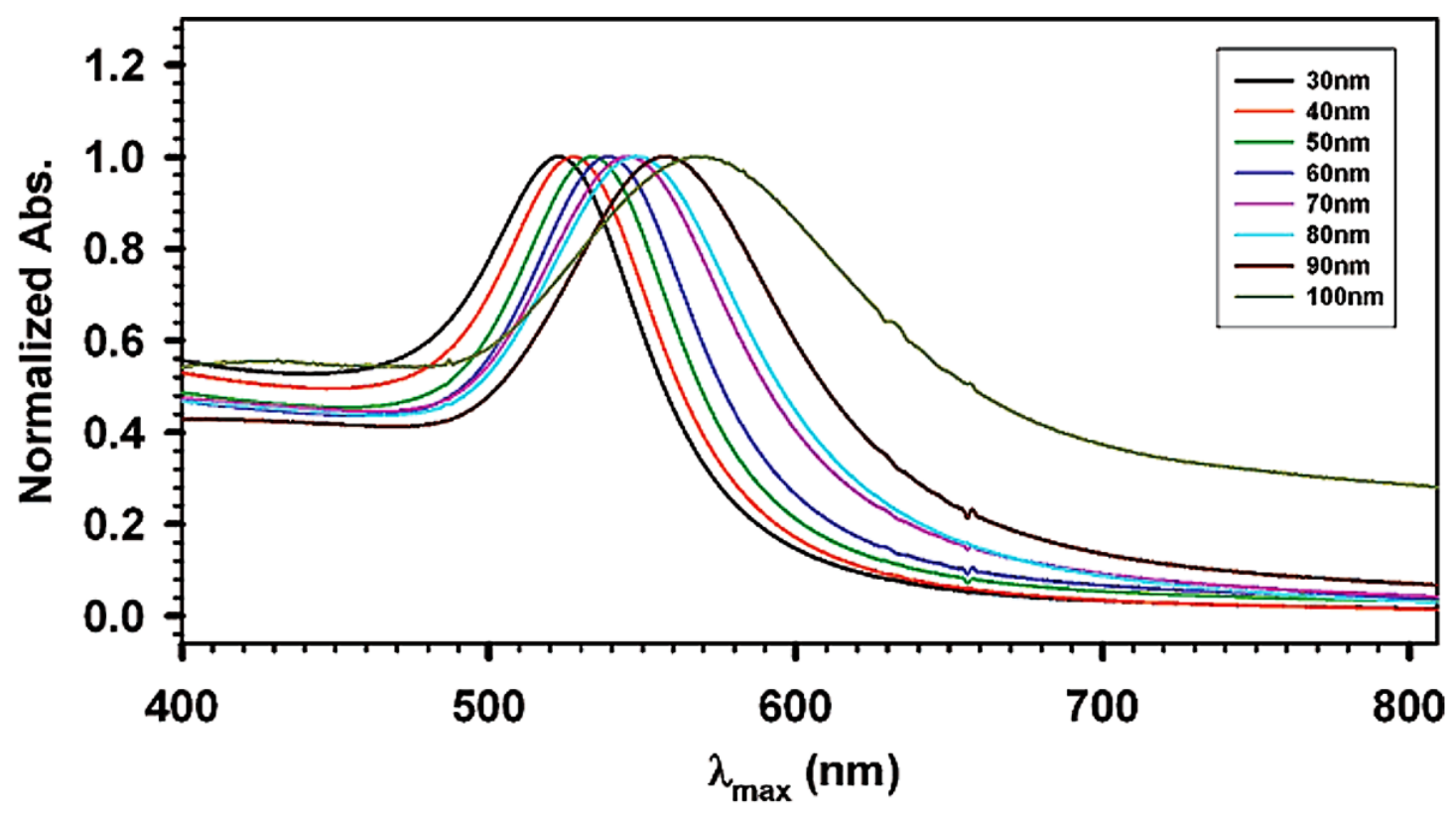

Figure 28: Normalised UV-Vis spectra for Au nanoparticles with different sizes in aqueous solution. Reprinted with permission from Njoki et al. Copyright 2007. American Chemical Society.

Another important aspect of the characterisation is the charge of the nanoparticles, which indicates how stable the colloid is in terms of how much the nanoparticles repel one another. This was important for the current study as the colloidal solutions were required to remain the same as the coating process occurred, without the nanoparticles agglomerating and precipitating out of solution as this would have cause uneven coverage of the fibre. Physical characterisation of the system was able to give an insight into the nature of the gold nanoparticular colloids by means of UV-Vis spectroscopy, zetasizing, microscopy and $\mathrm{pH}$ testing. This characterised the system in terms of particle size, dispersity, concentration and charge, enabling further investigation into the properties of the system by way of computational analysis and SERS.

\subsubsection{Computational Chemistry}

Computational chemistry allows one to model potential reaction mechanisms and to determine the optimal geometries, energies and frequencies of components within a reaction, amongst other properties. By knowing the optimised configurations and energies of the molecules involved in the gold-thiol interactions, it was possible to model theoretical spectra, in particular Raman spectra.

In 1999 Schlegel and Halls computed the theoretical Raman intensities for a variety of small molecules (including $\mathrm{H}_{2} \mathrm{O}, \mathrm{C}_{2} \mathrm{H}_{4}, \mathrm{H}_{2} \mathrm{~S}, \mathrm{SiO}_{2} \& \mathrm{CH}_{2} \mathrm{Cl}_{2}$ ) and tested three different basis sets to see which gave values closest to those experimentally determined. ${ }^{46}$ They 
compared Hartree-Fock, second-order Moller-Plesset perturbation theory (MP2), and density functional theory (DFT), including local, gradient-corrected, and hybrid functionals (S-VWN, B-LYP and B3-LYP, and MPW1-PW91). Comparisons were also made between three different basis sets $(6-31 \mathrm{G}(d)$, Sadlej, and aug-cc-VTZ) and it was found that the quality of basis set used was the most important feature in gaining concordant results. ${ }^{46}$ They found that DFT (using B3-LYP) in combination with the midsized Sadlej basis set gave results that were time effective and yielded excellent quantitative results. ${ }^{46}$ However, from the results in their research it was noted that 6-31G $(d)$ was the least computationally expensive and for the scope of the current research a DFT B3LYP functional with 6-31G and aug-cc-pVTZ/aug-cc-pVTZ-PP basis sets were used in the hope that these calculations would aid in the optimisation of the SERS experiments quickly and with reasonable accuracy.

\subsubsection{Microscopy Techniques}

Microscopy techniques were used to analyse the plausibility and origin of any contamination throughout the SPME extraction process (as previously detailed in Figure 18) and where and what the contaminants may be. Transmission Electron Microscopy (TEM) was used to characterise the gold colloids in solution to determine particle size, shape, composition, and dispersity. TEM is particularly suited for this type of analysis, which occurs on an almost atomic level in an imperfectly crystalline system. ${ }^{47}$

Alternatively, a Scanning Electron Microscope (SEM) can also be used to determine the shape, size, and coverage of the nanoparticles on the fibres. SEM can also be used in back scatter mode where high-energy electrons are reflected off the sample. Heavy elements, with high atomic numbers, backscatter electrons more effectively than lighter elements and therefore, appear a lot brighter in the image. This mode is used to detect contrast between areas with different chemical compositions. ${ }^{48}$ Back scatter mode was able to show that the nanoparticles are made of an element much heavier than components of the fibre and led to Energy-Dispersive X-ray Spectroscopy (EDS), which is an analytical technique that is used for elemental analysis and the chemical characterisation of a sample. It relies on the interaction between the X-ray excitation of a sample and its capabilities which are primarily due to the fundamental principle that each element has a unique atomic structure allowing for a unique set of peaks within its X-ray spectrum. This would confirm the presence of gold nanoparticles and subsequently which elements were nearby (and therefore likely to be bound to them), or how they change during thiol 
adsorption and desorption. Unfortunately, many elements will have overlapping peaks that will reduce the accuracy of some measurements. ${ }^{48}$

\subsubsection{Surface-Enhanced Raman Spectroscopy}

Alternatively, SERS could be used to analyse the surface as it is a surface-sensitive method that uses the Raman scattering of molecules attached to metal surfaces. SERS uses local field enhancements such as the surface plasmon resonance effect of metals to increase the signal caused by Raman scattering of molecules that are attached to the surface, or very near to it. ${ }^{49}$ To this end, it was possible that thiols adsorbed to a nanoparticle surface would be detected as Raman spectroscopy can differentiate between C-S bonds, S-H bonds and R-SH bonds. ${ }^{50}$ The thiols of interest are not particularly good Raman scatterers however, as they do not have many lone pairs or double bonds, which have been found to give the strongest Raman signals. Fortunately, if a molecule is not a good Raman scatterer the signal can be enhanced by using a substrate with a better enhancement factor, larger surface area or the laser power/scattering area could be increased. Typically the former is the approach of choice as more effective substrates are less limited by instrumental conditions. ${ }^{49}$ There are three main classes of substrates that can be used and altered:

1. Metallic particles e.g. colloidal nanoparticle solutions, typically of gold or silver.

2. 'Planar' metallic structures e.g. arrays of nanoparticles on planar substrates.

3. Metallic electrodes.

The benefit of using SERS, as opposed to Raman, is caused by plasmon resonance that can cause varying levels of amplification throughout the spectrum. ${ }^{49}$ SERS is a highly specific technique as it gives a molecular fingerprint, thus making it easier to distinguish a molecule from background signals and noise. It can be directly applied to any molecule without need of a fluorophore or other type of indicator. ${ }^{49}$ Another key difference is a secondary effect caused by the surface selection rules and can occur when a molecule adsorbs to the surface of the substrate and forms a surface complex. This is advantageous as this change can result in small shifts or broadening of the peaks and in some extreme cases peaks may appear, move or disappear altogether, which can give information relating to the surface binding. ${ }^{49}$ 
The Raman cross sections may also change, which is often called the chemical enhancement effect. ${ }^{49}$ The cross section of the molecule $(\sigma)$ for a known linear optical process relates the signal produced by this process, characterised by its intensity or power (P) relative to the incident power density $\left(\mathrm{S}_{\text {Inc }}\right)$ at the molecule position as:

$$
P=\sigma S_{I n c}
$$

The cross section of a molecule depends on the wavelength used. The cross section applies to a single molecule, even if many molecules are involved in the experiment, which makes it slightly difficult to measure (but not impossible). In general terms the larger the cross section, the stronger the interaction is and therefore the more likely the 'collision' is to occur and give a signal. ${ }^{49}$

There are many factors that affect the enhancement that can be provided by SERS and the enhancement factor values can range from approximately 10 to $10^{15}$. These large differences are typically due to changes in substrates but even within the same substrate there can be a difference of a few orders of magnitude. ${ }^{49}$ There are two main contributors to the enhancement;

1. Electromagnetic - To succumb to the effect of this enhancement the molecule must be close to the surface (within $\sim 10 \mathrm{~nm}$ at most). This is the main contributor, it relies on the local field enhancement close to the metallic surface when localised surface plasmon (LSP) resonances are excited. $^{49}$

2. Chemical - This enhancement can induce resonant-Raman scattering at wavelengths where the non-adsorbed molecules would not be resonant. The most likely explanation for this is charge transfer, which requires the molecule to be chemically adsorbed to the surface. ${ }^{49}$

At the same wavelength, most molecules provide a similar spectra in SERS as they do in Raman and the fingerprint Raman peaks are typically identifiable for each molecule. ${ }^{49}$ This means that not only is it possible to gauge the interactions between the thiols and the gold, but could also identify possible contaminants in the system. By theoretically predicting the Raman spectra of the compounds, it is possible to find the appropriate regions to analyse in a SERS spectra and look for similarities and differences in the spectra produced during the different stages of the analytical process (adsorption, desorption and potentially after any cleaning procedures during the protocol). 


\subsection{Results and Discussion}

\subsubsection{Microscopic and Physical Characterisation}

Colloidal gold nanoparticle solutions

Table 6: Comparison of calculated were prepared and prior to coating a fibre, they were analysed. TEM analysis d-spacing and reference values for gold.

(solution was drop cast onto a copper $\begin{array}{lllll}\text { Ring } & \text { d }(\AA) & \text { d }(\AA) & {[h k l]} & \text { Intensity }\end{array}$ No. measured gold $^{51}$ grid) showed the diffraction pattern of the nanoparticles (Figure 29). The calculated d-spacings match the

\begin{tabular}{ccccc}
\hline \hline A & 2.395 & 2.355 & 111 & 100 \\
$\mathbf{B}$ & 2.027 & 2.039 & 200 & 52 \\
$\mathbf{C}$ & 1.450 & 1.442 & 220 & 32 \\
$\mathbf{D}$ & 1.245 & 1.23 & 311 & 36 \\
$\mathbf{E}$ & 1.054 & 1.1774 & 222 & 12 \\
$\mathbf{F}$ & 0.958 & 1.0196 & 400 & 6 \\
\hline
\end{tabular}
reference values for gold nanoparticles and the facets available for reactions.

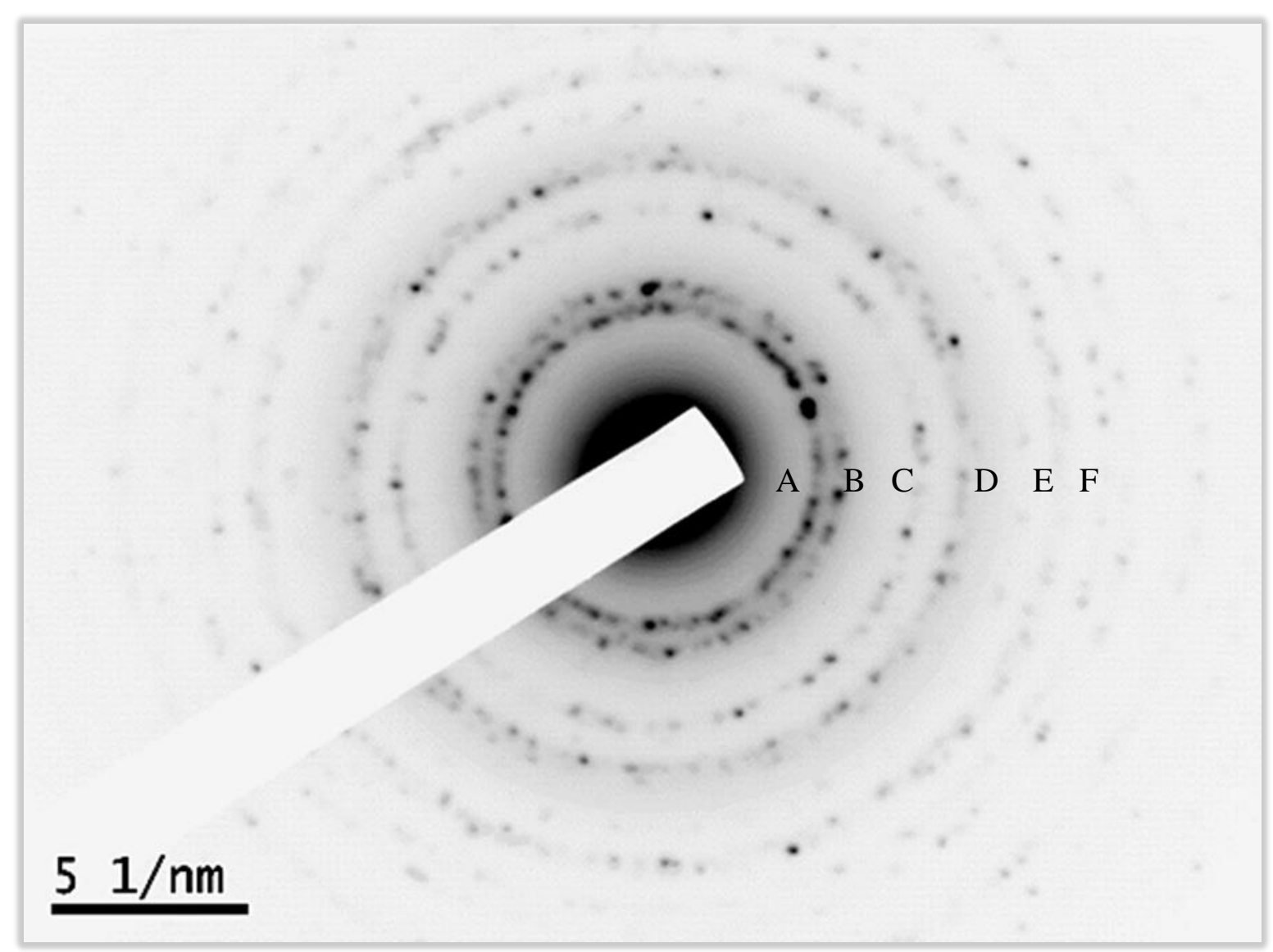

Figure 29: TEM diffraction pattern of gold nanoparticles (inverted colours). 
The colloidal gold solutions were wine-red in colour once formed, however, during the synthesis they appeared a much darker burgundy in colour. The solution (Figure 30- left) was used in the TEM analysis and then diluted by a factor of six until it was translucent pink in colour for use in further physical characterisation.

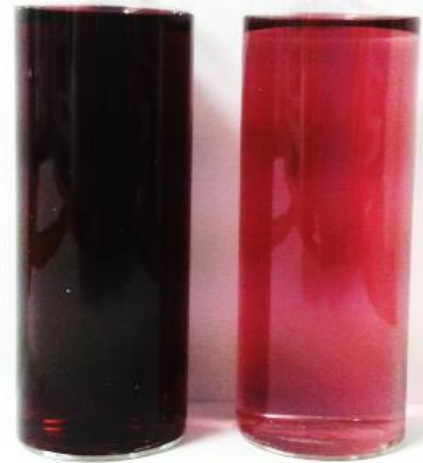

Figure 30: Concentrated (left) and diluted (right) colloidal gold samples.

A low resolution image of the sample (Figure 31) indicates that the majority of gold nanoparticles in the sample were well dispersed and approximately $25 \mathrm{~nm}$ in diameter and that there are a few slightly larger nanoparticles at $\sim 40 \mathrm{~nm}$. The inset high resolution picture in Figure 31 also shows the lattice fringes the nanoparticle, these were calculated to be $4.1667 \AA$ which is indicative of gold. ${ }^{51}$

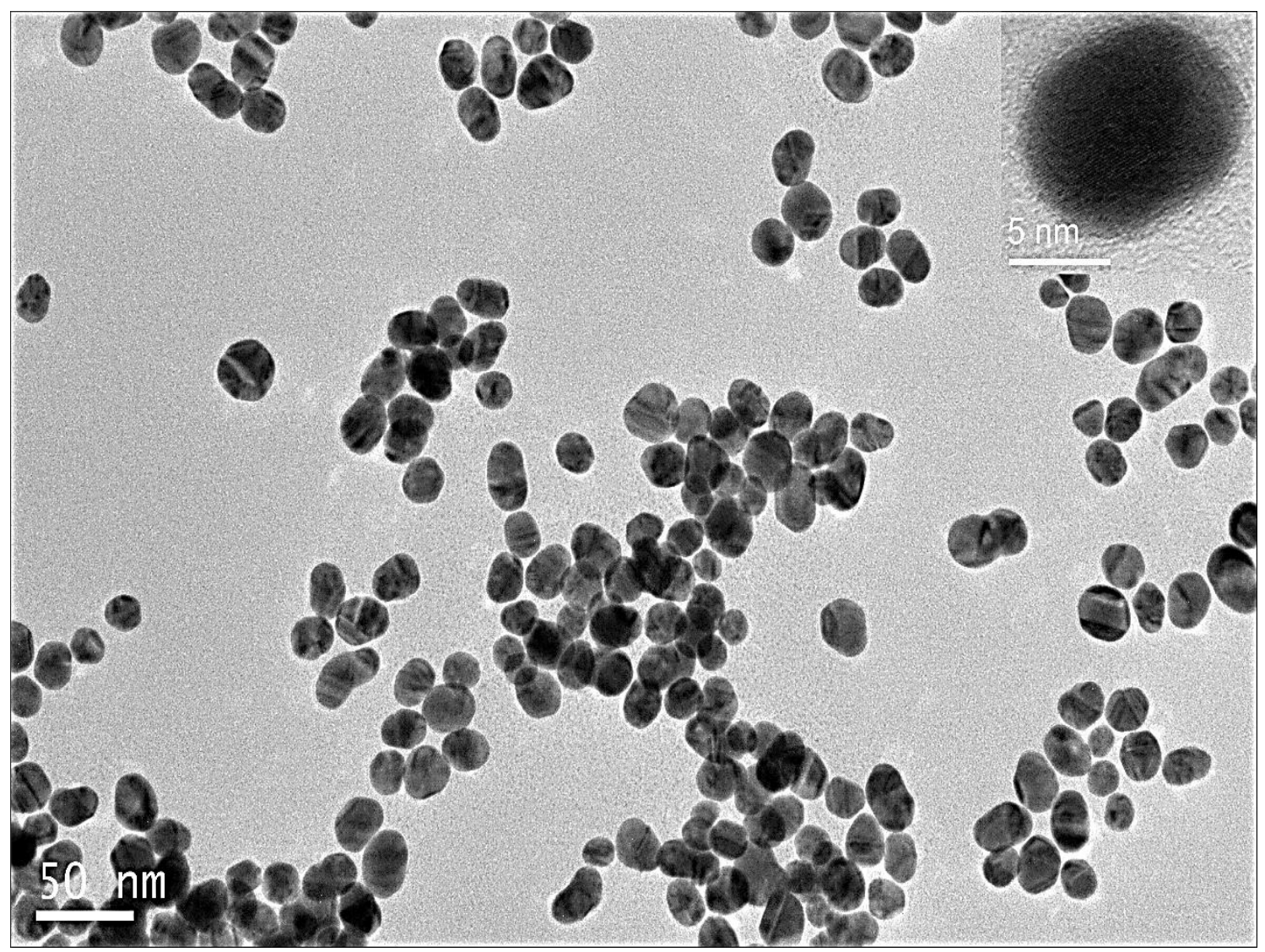

Figure 31: TEM image of colloidal nanoparticles drop cast on a copper grid. 
An EDS map was then taken to show that the gold nanoparticles were pure and that there were no contaminants in the system. The characterisation of the gold nanoparticles using electron microscopic techniques verified that the nanoparticles formed were indeed gold and that the synthesis employed rendered nanoparticles that while polydisperse in size, there were a large number of single nanoparticles that had not agglomerated in solution.
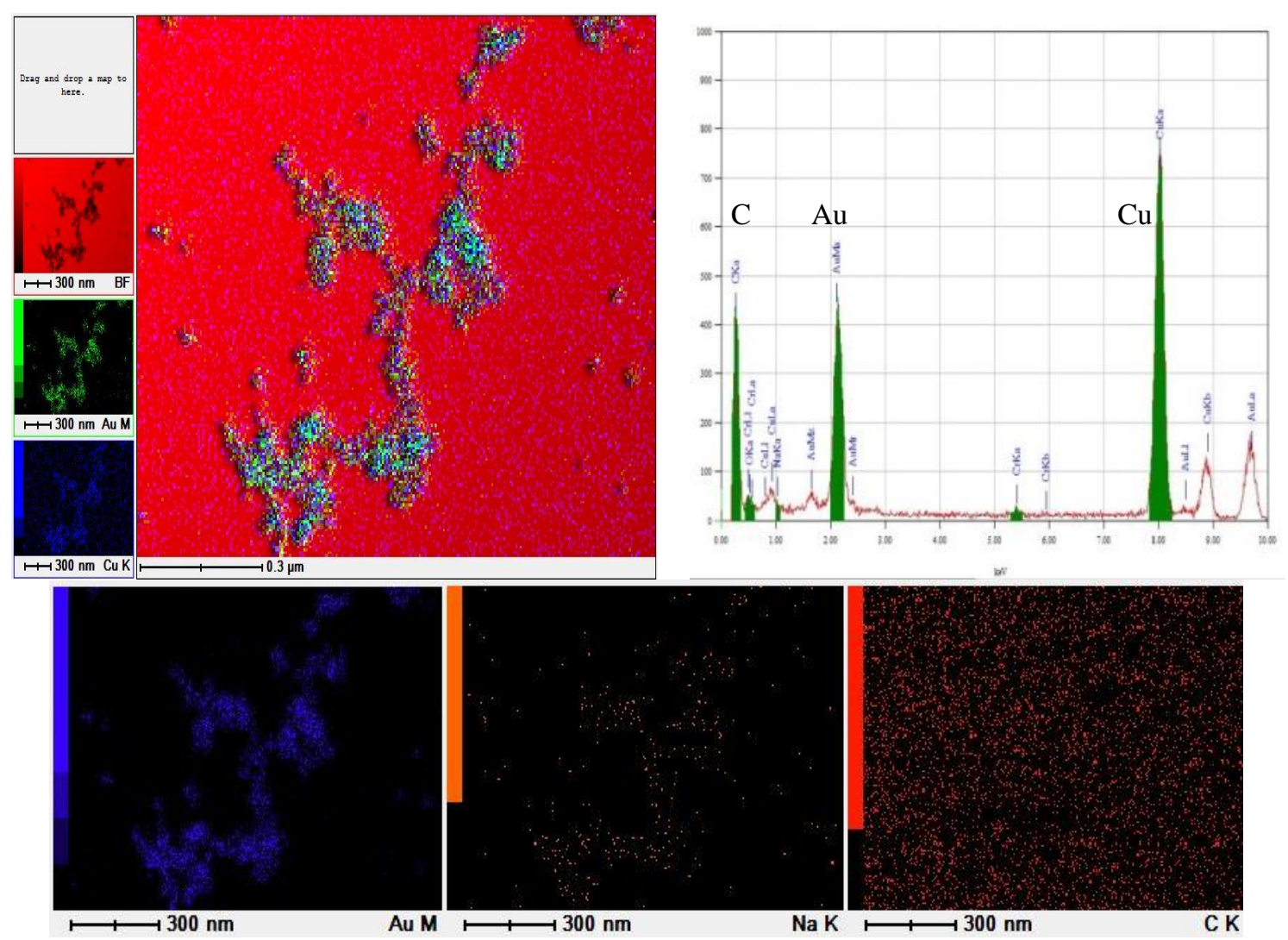

Figure 32: EDS images of colloidal gold nanoparticles. Top left: Overlay of elemental traces, top right: spectral elemental analysis, bottom: individual elemental traces.

Due to time constraints, the EDS maps taken of the evaporated liquid samples were only taken with a minimum number of scans in order to get an indicative result as high resolution was not deemed of high importance for these samples above being able to see the composition of the nanoparticles. It can be seen by spectral analysis that the most abundant atoms in the sample are copper, gold and carbon (Figure 32). This is to be expected as the colloidal gold samples were suspended on a copper grid for analysis and therefore, there is an abundance of copper in the vicinity of the sample. The gold nanoparticles were synthesised using an organic reducing and capping agent (TSC) which is believed to be the cause of the high proportion of carbon seen throughout the sample. This is further thought to be the case due to increased levels of sodium atoms near the gold nanoparticles in comparison to the rest of the area. It is possible that the dissociated TSC was in excess during the nanoparticle synthesis and that the excess citrate molecules 
then dispersed throughout the colloid where they could hydrogen bond with the solvating water molecules. The sodium atoms on the other hand may remain associated with the nanoparticles due to the negative 'shell' that formed by the capping citrate molecules.

Physical characterisation of the colloids was then pursued, the results indicated that the $\mathrm{pH}$ of the colloids was approximately 5.4. In comparison to the $\mathrm{pH}$ of the triple distilled deionised water used for sample preparation, this is very low which suggests that there was still some TSC dispersed throughout the colloid giving a slightly acidic solution. This in turn would indicate that the gold nanoparticles were fully coated with TSC which further supports the abundance of carbon seen throughout the colloid (Figure 32). A UV-Vis spectrum was obtained for the colloids to determine their size (Figure 33). It was found that the $\lambda_{\max }$ of the colloids was $526 \mathrm{~nm}$, which is indicative of 20-30 nm diameter nanoparticles. ${ }^{45}$ However, the peak was quite broad which suggests that there is a degree of polydispersity in the sample.

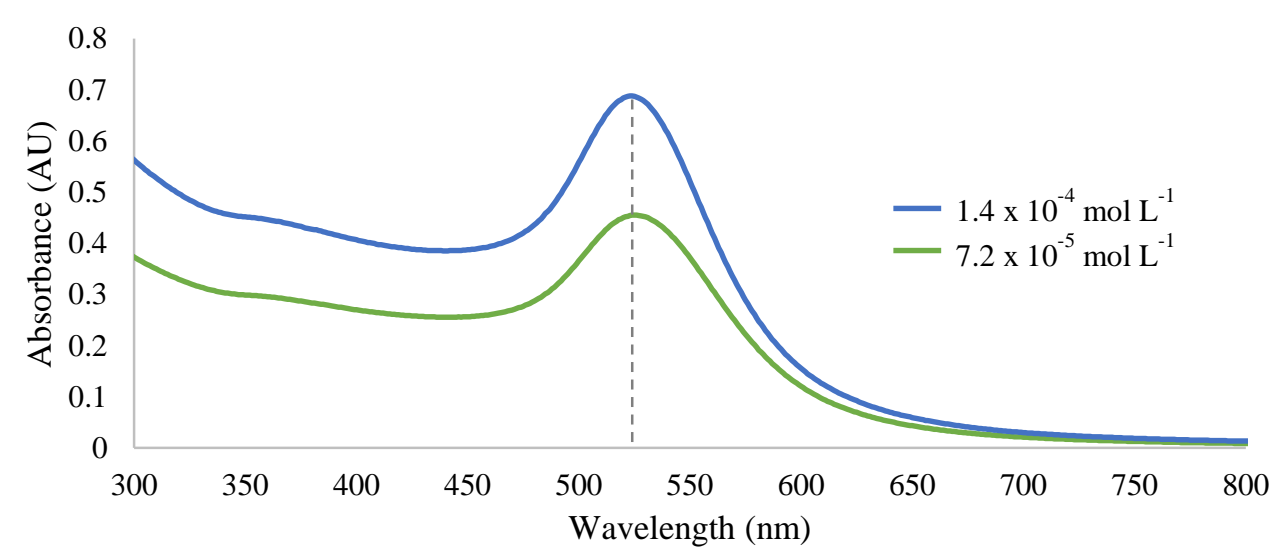

Figure 33: UV-Vis spectra of colloidal gold nanoparticle solutions.

The colloids were then tested in a zetasizer to determine the size and charge of the nanoparticles. It was found that the colloids were indeed polydisperse, there was a large degree of nanoparticles that were very small $(\sim 20 \mathrm{~nm})$ but a very small number were larger $(\sim 40 \mathrm{~nm})$ (correlating with Figure 31), giving a greater contribution towards the intensity which agreed with the TEM and the UV-VIS data. The zeta potential of the colloids was found to be $-27.8 \mathrm{mV}$ that indicated that the particles are repelling each other and therefore the colloid is reasonably stable. This could also be seen in the laboratory, as the colloids would retain their colour, viscosity and translucency for months after their formation. 
To visualise the gold nanoparticles that were coated onto the SPME fibres required a model system, in this case, glass fibres (primarily comprised of silica) were used in place of the silica SPME fibres as SEM preparation would destroy the usable SPME fibres. The glass fibres were then analysed using SEM and showed that the gold nanoparticle coating on the fibres was replicated (in relation to previous research). ${ }^{41}$ It can also be seen that the nanoparticles were evenly distributed on the fibre and that the average size and dispersity of the nanoparticles was retained between the colloid and the fibre coating (Figure 34a). The back scatter image indicates that the particles on the surface of the fibre were of a higher molecular weight than the glass fibre (Figure 34b) and via EDS analysis it was established that the nanoparticles were gold.
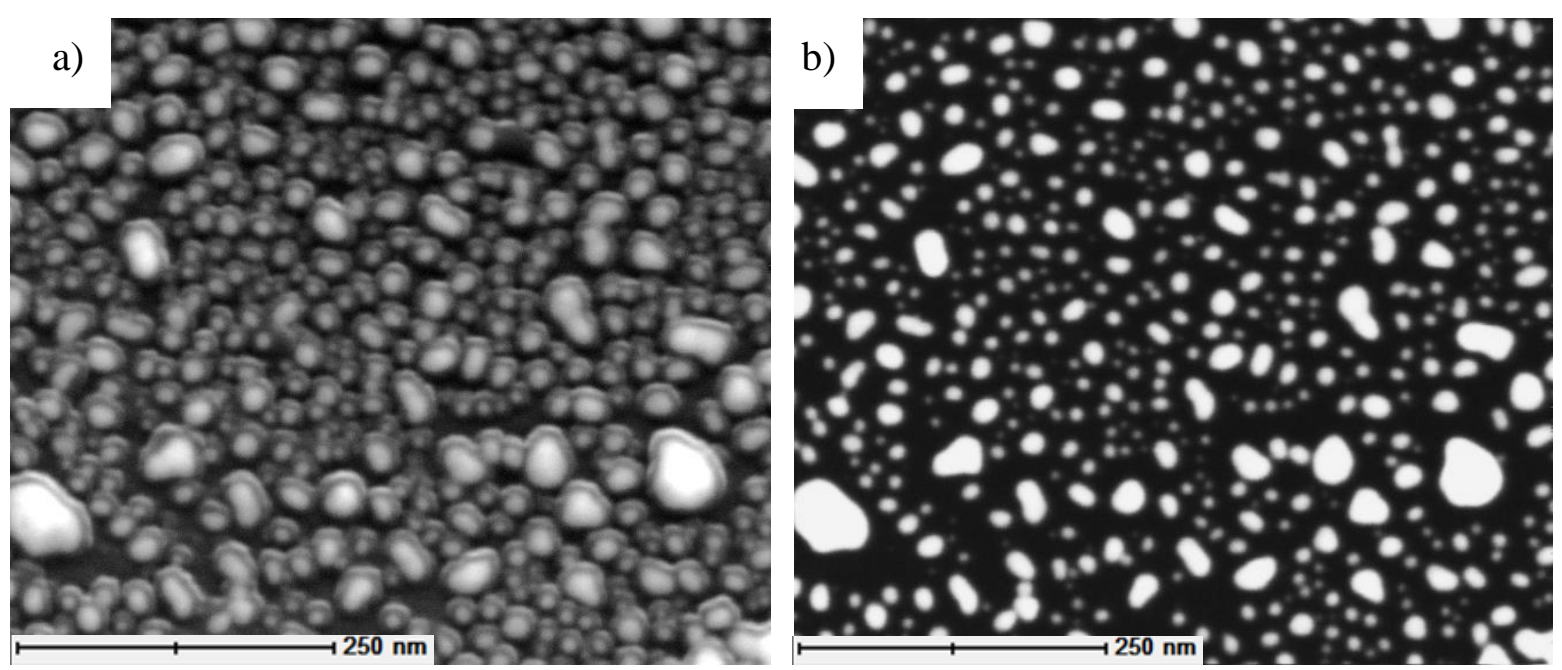

Figure 34: a) SEM of gold nanoparticles b) Backscatter image of gold nanoparticles.

It can also be observed (Figure 34a) that the nanoparticles appear to have a thin 'shell' around the gold core. Initially, this was thought to be caused by the conditioning of the fibres and that the outer core was slightly 'melted' by this process. However, by backscatter analysis (Figure 34b) it would appear that the high mass areas have larger spaces between them than envisioned by the SEM picture. This indicates that the inner core has a much higher molecular mass than the shell. The shell does not visibly appear in the backscatter image which would suggest that its components are of a much lower atomic weight than the gold and that it may consist of elements that are similar in mass to other potential surrounding molecules (Si fibre, APTMS polymer or TSC) which were used in the synthesis of the nanoparticles and their subsequent bonding to the fibre.

Further images were taken in order to try to identify possible contaminants during the mock run of an injection using the glass fibres. Images were taken of the fibres before and after being coated with the gold nanoparticles, once the fibre was conditioned, after 
thiols adsorption, and again after desorption. Analysis of all images and subsequent EDS maps showed no potential contaminants binding to the surface of the gold which supports the results found during the Protocol Standardisation (page 30).

Furthermore, while by odour it was noted that excess thiols were in the vicinity of the surface, it was impossible to determine whether the sulfur had bound to the nanoparticles following thiol headspace extraction via SEM analysis. Unfortunately gold and sulfur peaks overlap in the EDS spectral analysis (Figure 35) and other gold peaks we could have

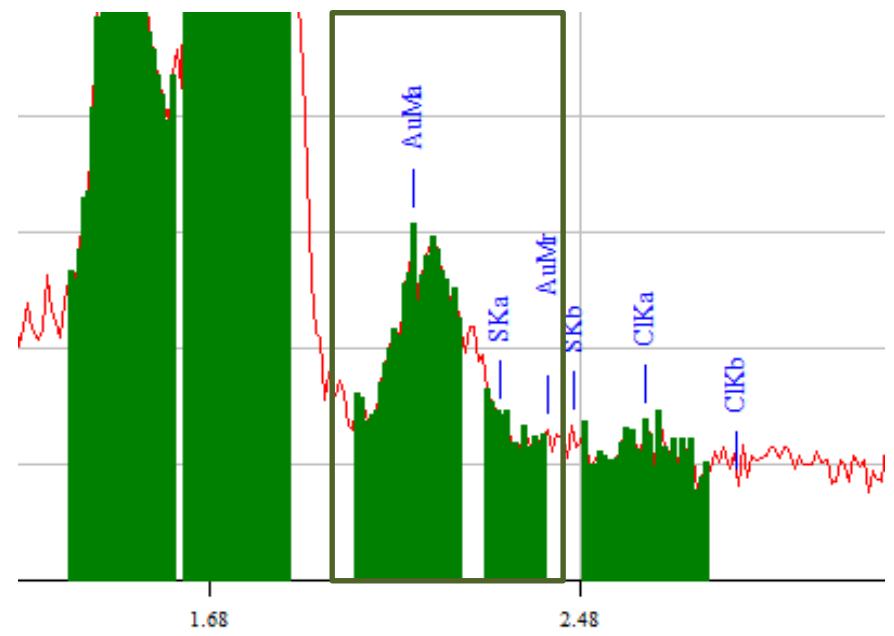

Figure 35: Spectral analysis highlighting Au, S overlap.

seen were too small to provide a decent map. Therefore although it appears that there are some regions where sulfur and gold are present, it cannot be proven that the results are not a false positive.

Being able to characterise the gold nanoparticles led to the need to further identify the electronic and steric properties of the thiols and the possible orientations in which they bound to the gold as well as the mechanism by which this occurred, in order to further control the process. Consequently, attempts were made to analyse the gold surface prior to adsorption and after desorption. To do this, predicted Raman spectra were obtained using computational analysis. 


\subsubsection{Computational Chemistry}

Computational approaches were used to simulate the binding of the wine thiols to gold surfaces in order to probe the steric and electronic properties of the bound thiols and the gold surfaces.

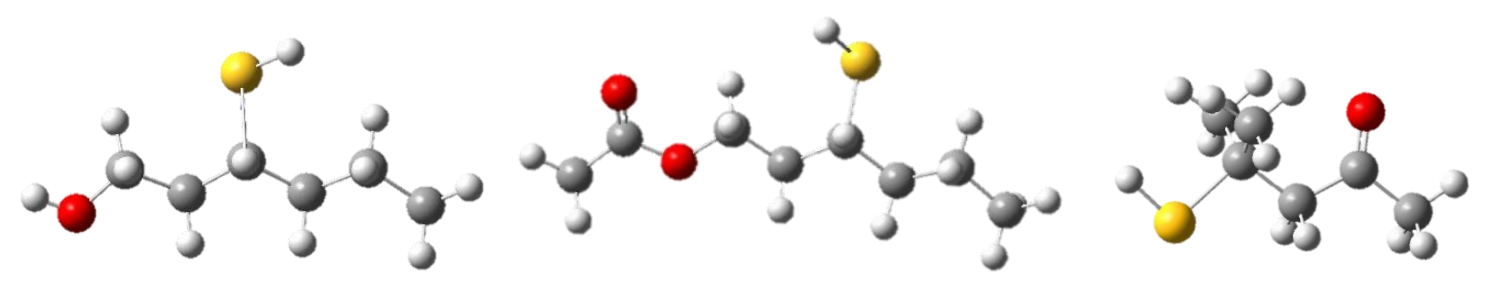

Figure 36: Optimised geometries of three thiols: (left to right) 3SH, 3SHA and 4MSP.

The optimised structures of the free thiols predicts theoretical similarities in the bond length and charge of the C-S segment, particularly in the case of 3SH and 3SHA. These similarities are also seen in the modelled length of the S-H bond in the molecules where in all three thiols the bond length is $1.36 \AA$. The estimated atomic charge of the atoms involved in the thiol functionality indicates a marginal difference between the three thiols; in $3 \mathrm{SH}$ and 3SHA the charge on the sulfur atom is $-0.51 \overline{\mathrm{e}}$ whereas in 4MSP the charge is $-0.56 \overline{\mathrm{e}}$. However, the sulfur bound protons were all $0.04 \overline{\mathrm{e}}$. The charges on the sulfur bound carbon however, are all reasonably different; 3SHA has the carbon with the lowest theoretical charge $(0.36 \overline{\mathrm{e}})$ as the acetyl group is likely to be drawing electron density from the rest of the molecule. The withdrawing effects of the alcohol (S-C* $-\mathrm{R}$ charge $=0.41 \overline{\mathrm{e}})$ and ketone $\left(\mathrm{S}-\mathrm{C}^{*}-\mathrm{R}\right.$ charge $\left.=1.22 \overline{\mathrm{e}}\right)$ groups in the other two thiols are much less pronounced. The tertiary sulfur bound carbon in 4MSP is likely to have a greater charge as it is bound to an extra methyl group instead of a proton. From this it can be seen that while the three thiols are very similar in nature, there are some key differences that should make them distinguishable on the surface during characterisation.

Another area that was theoretically explored was the surface area the thiols occupied and if the surface was being fully covered before the supply of analyte was exhausted thus giving irreproducible adsorption and desorption. It was estimated that one molecule of $3 \mathrm{SH}$ had an approximate area of $1.72 \times 10^{-7} \mathrm{~cm}^{2}$ and that $7.33 \times 10^{5}$ molecules would be required to cover a gold coated fibre with an estimated surface area of $0.126 \mathrm{~cm}^{2}$ (in a simplified model of the system). The samples used in the analysis were typically of a concentration of $50 \mu \mathrm{g} \mathrm{L}^{-1}$ and $10 \mathrm{~mL}$ of sample was used in each analysis. This means that in each sample there was $2.24 \times 10^{21}$ molecules and therefore all binding sites can 
potentially be occupied as there is an excess of target analyte. This suggests that the reproducibility of the protocol relies on all of these sites being available from binding, consequently the understanding of the binding reactions at the surface were critical to further development.

\subsubsection{Surface-Enhanced Raman Spectroscopy}

Theoretical calculations of the thiols and their binding properties with gold led to the possibility of using SERS to examine these interactions. Once the thiols were optimised using Gaussian software, it was possible to predict their likely Raman spectra (Figure 37), and when bound to gold, changes in the calculated spectra were observed (Figure 38). Using the spectra obtained in combination with the nanoparticles' characteristics (size, shape, charge and concentration), it was possible to calculate the Raman cross section of the molecules and therefore analyse them using SERS.

As can be seen in the predicted Raman spectra (Figure 37), the three thiols are very similar in nature but there are some distinguishing peaks, 3SH can be distinguished from 3SHA and 4MSP by an O-H stretching band near $3600 \mathrm{~cm}^{-1}$. Similarly, 3SHA is unique with the ester OAc band near $1100 \mathrm{~cm}^{-1}$, while 4MSP can be differentiated from the other two by several unique bands between 50 and $1000 \mathrm{~cm}^{-1}$ (Figure 37).

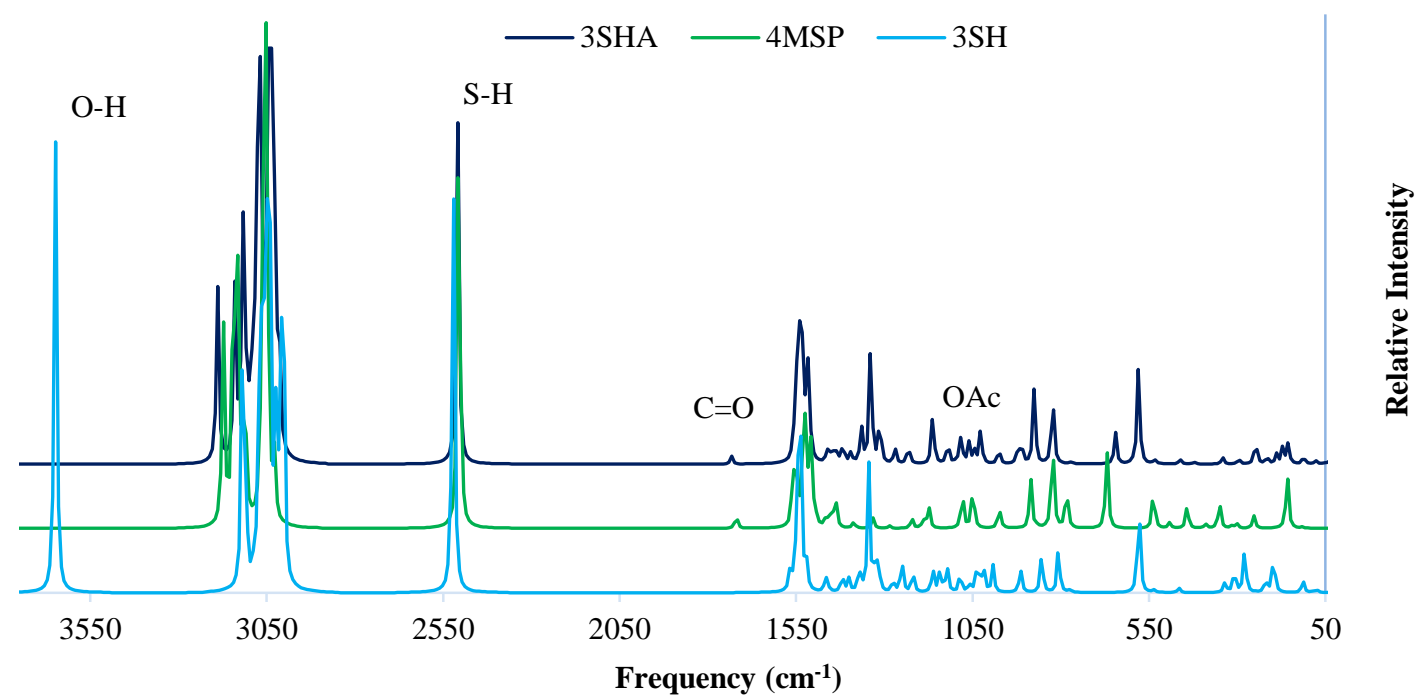

Figure 37: Predicted Raman spectra of 3SHA, 4MSP \& 3SH.

In order to model the spectra that might result from SERS, 3SH was modelled bound to a single gold atom and the structure was optimised. From this optimised model a vibrational calculation was run to give Figure 38. While this was not entirely representative of a gold nanoparticle surface, due to time constraints and computational 
costs involved with modelling large systems with copious electrons, clusters of gold atoms were not a viable modelling option.

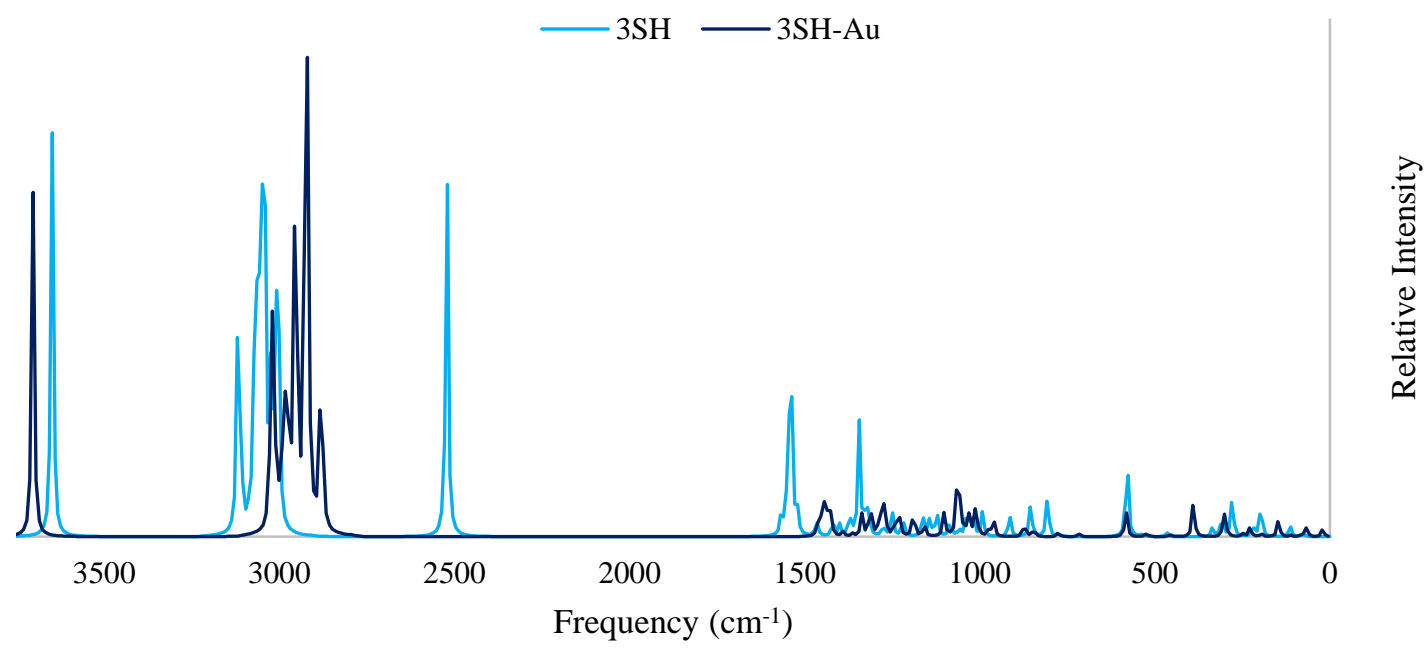

Figure 38: Predicted Raman spectra of 3SH and 3SH-Au.

In the predicted spectra (Figure 38) it can be seen that binding sulfur to the gold causes a slight shift in the peaks and in the region above $2000 \mathrm{~cm}^{-1}$ there is a slight enhancement of the predicted peak intensities of the $\mathrm{C}-\mathrm{H}$ stretching. The other noticeable change was the disappearance of the peak at $2500 \mathrm{~cm}^{-1}$, attributed to the $\mathrm{S}-\mathrm{H}$ bond, which is no longer present in the gold bound complex.

Colloidal gold and $3 \mathrm{SH}$ solutions were made up to $3 \mathrm{~mL}$ in cuvettes with varying ratios between the nanoparticles and thiols these samples were then subjected to the SERS laser to produce a spectrum ( Figure 39). The experimental limits of the SERS instrument were set to between 600 and $1600 \mathrm{~cm}^{-1}$ in order to monitor the differences in organic stretches and bends between spectra of the colloidal gold nanoparticles (coated in TSC) and those that had been spiked with varying concentrations of thiols. As the thiols were expected to be bound to the gold, it was unlikely that there would be an SH peak to observe at higher wavelengths, however, if in the unlikely event of unbound thiol being present in amounts great enough to be detected by SERS, a peak at $900 \mathrm{~cm}^{-1}$ was expected due to a SH bend. While gold will not produce a SERS spectra alone, the nanoparticles are capped with TSC which has the potential to do so. This being the case, the displacement of TSC by thiols on the surface of the gold nanoparticles (Figure 39) appears to be favoured. 


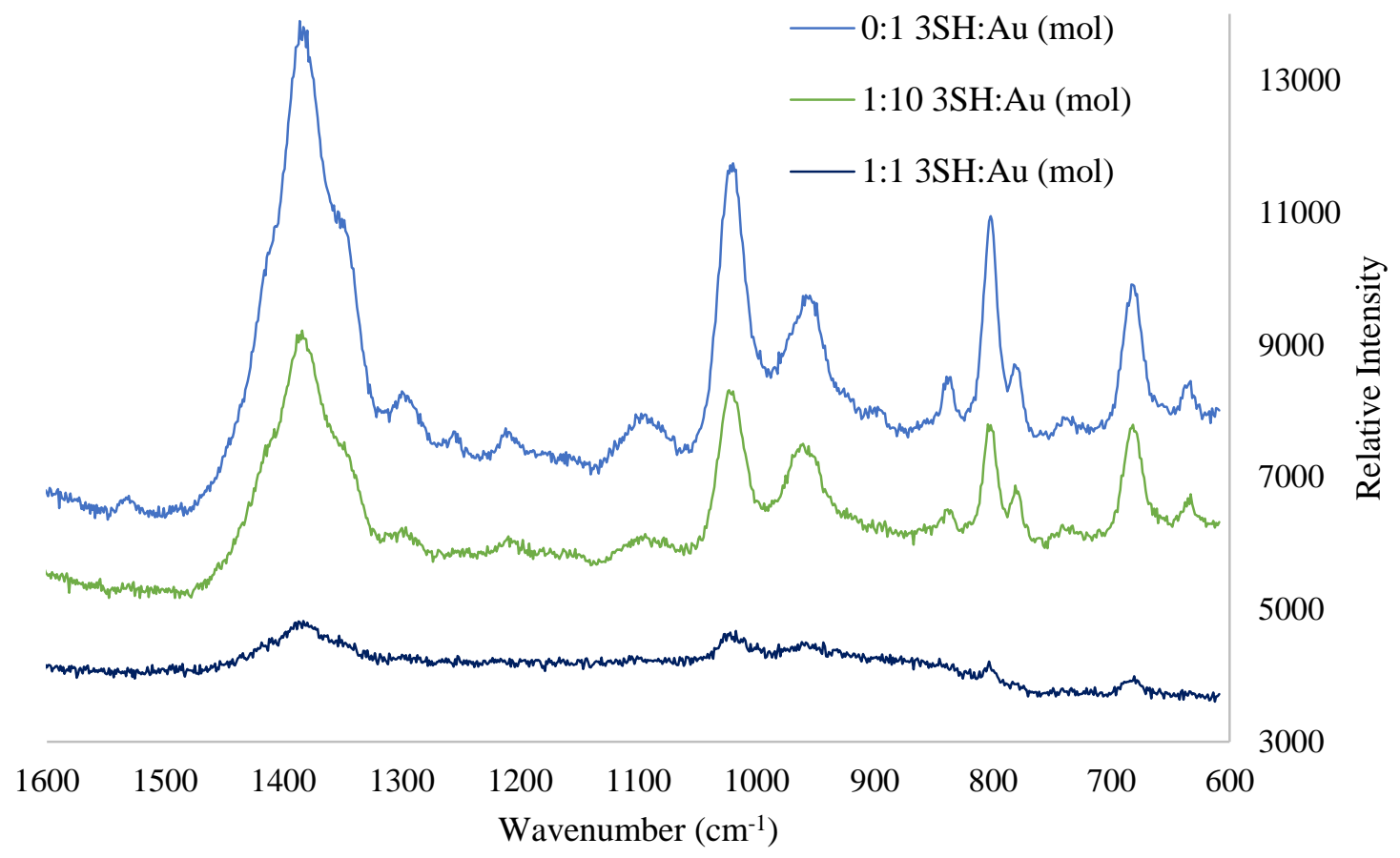

Figure 39: SERS spectra of varying concentrations of thiols in a gold colloid.

This data shows that as increasing amounts of thiol are added to the colloidal gold, the major peaks in the SERS spectra diminish and when the molar ratio is $1: 1$ the citrate is almost completely displaced by the thiol. It is hypothesised that because $3 \mathrm{SH}$ is not particularly Raman resonant, there needs to be a much higher concentration in the colloid for their characteristic peaks to be visible above the noise of the spectrum. Therefore, while this avenue of surface visualisation appears promising, due to time constraints and machine malfunctions, further analysis was not possible during current research project. 


\section{Binding Reactions}

\subsection{Introduction}

The chemical reaction that takes place during adsorption involves a thiol binding to a gold surface, typically requires the removal of the hydrogen from the sulfur, indicating an oxidation of the neutral thiol to a thiolate with a charge of -1 (Scheme 6). This in turn implies that the gold nanoparticles, with a neutral charge, were reduced with each thiolate addition to the surface. All these chemical attributes needed to be taken into consideration in order to determine the cause of the hysteresis observed during initial experimentation. The way in which the thiols bind and orient on the surface was deemed to be important as well as the potential reaction mechanisms by which this occurs. These interactions were investigated both experimentally and computationally to interpret how they occurred and the possible reversibility of these reactions.

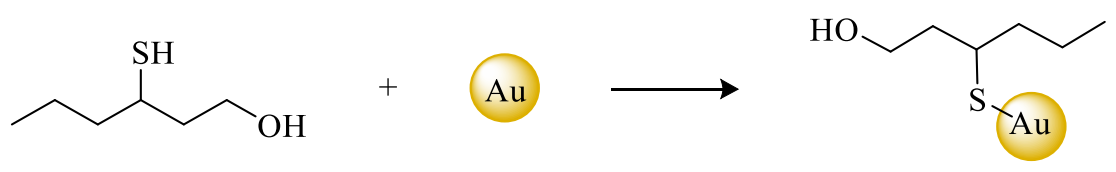

Scheme 6: 3SH addition to gold, demonstrating the thiolate-gold bond.

\subsubsection{Computational Chemistry}

The information provided by computational analysis can help predict the activation energy of a reaction and the overall energy change that takes place with bond breakage and formation, allowing conclusions to be drawn on the viability of a mechanism and the temperatures that are required for them to take place.

Previous research by Askerka et al. presented two possible mechanisms by which the hydrogen is removed from thiols as they bind to a gold atom or surface (Figure 40). ${ }^{52}$ Mechanism A indicates a staple motif and energy data has indicated that the introduction of a second thiol to the same binding site decreases the total energy required to remove the hydrogen from the thiol to form the thiolate bound to the gold by $4.6 \mathrm{kcal} \mathrm{mol}^{-1}$. Alternatively, mechanism B shows that as the thiols bind to a gold atom on a surface, the protons are shuttled to a neighbouring thiol and then on to the gold itself. This shuttling 
process detaches the proton from the thiol to form a thiolate and by proceeding in this manner, the energy needed to form a bond with the surface was theoretically decreased by $9.1 \mathrm{kcal} \mathrm{mol}^{-1} .^{52}$

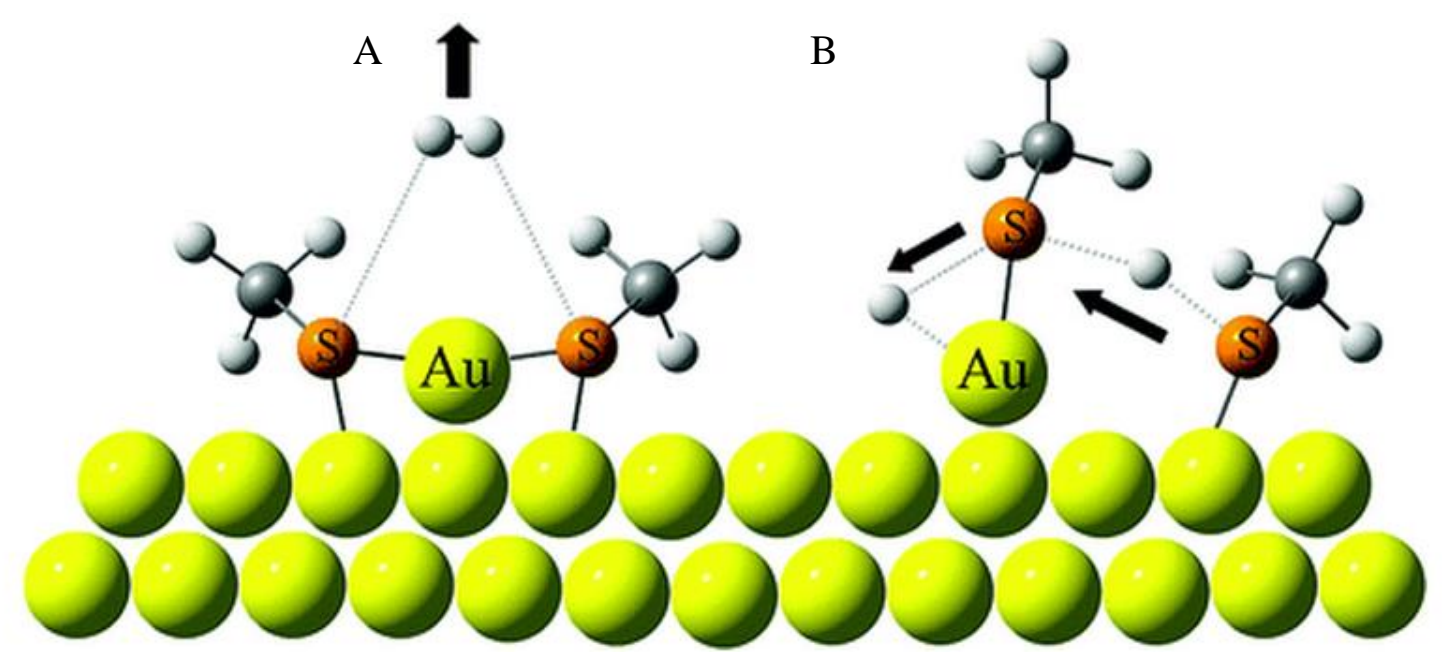

Figure 40: Two possible mechanisms for thiols binding to gold A- Staple motif B -Hydrogen shuttling. Reprinted with permission from Askerka et al. Copyright 2012. American Chemical Society. ${ }^{52}$

A similar approach could be used to probe the binding, oxidation, and reduction that occurs during thiol adsorption in the current research development. By using the optimised bond lengths and angles predicted by the Askerka study for the complexes, it was possible to lower the computational expense of the calculations. ${ }^{52}$ The bond lengths optimised for the molecules in the previous study were input as an estimated the initial geometry for the optimisations calculated in the current study. It was proposed that once the optimum geometries of the reactants and proposed products were determined, it would then be possible to determine transition states and intermediates of the reactions and hence the physical parameters by which the fibres could be cleaned for use in multiple analyses. 


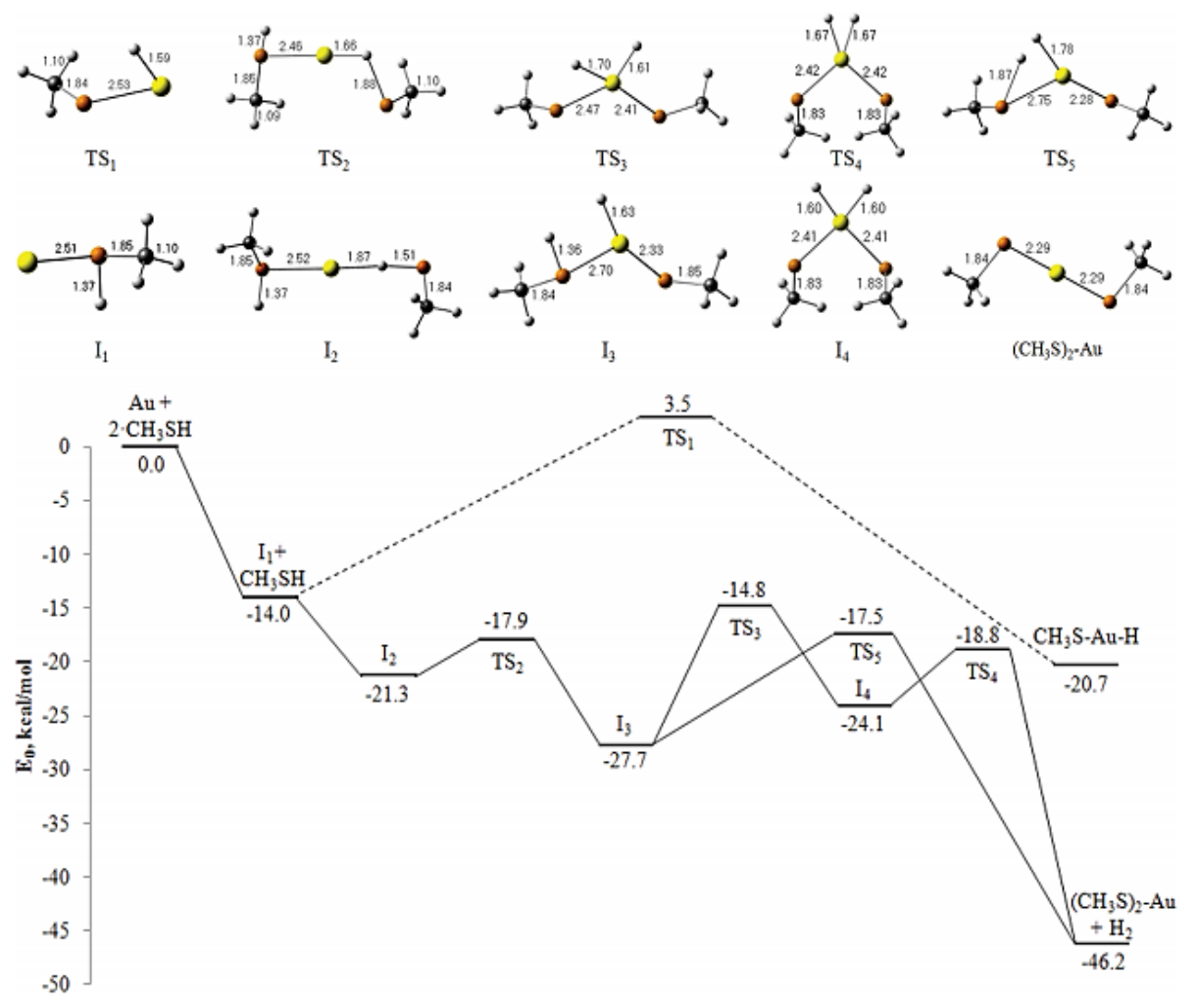

Figure 41: Optimised structures of reagents, products, intermediates $\left(I_{n}\right)$ and transition states $\left(\mathrm{TS}_{\mathrm{n}}\right)$ for the proposed mechanism of dissociative adsorption of methanethiol on a single Au. Reprinted with permission from Askerka et al. Copyright 2012. American Chemical Society. ${ }^{52}$

The mechanism proposed by Askerka et al. is shown in Figure 41and it can be seen that there is an overall decrease in energy for methane thiol binding to a single gold atom and an even bigger energetic decrease when two thiols are present and binding to the same gold atom. ${ }^{52}$ In the reaction where only one thiol is present, the transition state $\left(\mathbf{T S}_{1}\right)$ required to be overcome has an energy barrier of $17.5 \mathrm{kcal} \mathrm{mol}^{-1}$. A two thiol intermediate was also investigated and although there are more transition states and steps to proceed through, the maximum energy barrier to be surpassed is only $12.9 \mathrm{kcal} \mathrm{mol}^{-1}$ (TS3) and theoretically leads to a final product that is $25.5 \mathrm{kcal} \mathrm{mol}^{-1}$ lower in energy, making this second mechanism more plausible. A closer look at this mechanism indicates that the binding of methanethiol to the gold atom $\left(\mathbf{I}_{1}\right)$ has a binding energy of $14 \mathrm{kcal} \mathrm{mol}^{-1}$ and the binding of the second thiol ( $\left.\mathbf{I}_{2}\right)$ further lowers the energy by another $7.3 \mathrm{kcal} \mathrm{mol}^{-1}$. During this step it can be seen that the second thiol likely binds through the proton on the sulfur rather than through the sulfur atom. This would then lengthen the S-H bond in the second thiol as electron density is drawn towards the gold. As the suggested mechanism proceeds from $\mathbf{I}_{\mathbf{2}}$ to $\mathbf{T S}_{\mathbf{2}}$, the Au-H bond shortens and therefore, becomes stronger, while the $\mathrm{S}-\mathrm{H}$ bond lengthens and becomes weaker, this causes the $\mathrm{Au}-\mathrm{H}-\mathrm{S}$ bond angle to decrease (increasing the energy of the molecule) bringing the S closer to the Au. In $\mathbf{I}_{3}$, the 
$\mathrm{H}-\mathrm{S}$ bond has broken and the S becomes bound to the Au. This bonding is theoretically favoured in the system and the energy decreases by a further $9.6 \mathrm{kcal} \mathrm{mol}^{-1}$. After the third intermediate, it was predicted that there are two possible ensuing routes; the first (path a) involving three transition states and represents a step by step migration of the two protons from sulfur to gold (TS2, TS3) and the association of these protons to form $\mathrm{H}_{2}$ (TS4). The second (path b) in which there is a direct formation of $\mathrm{H}_{2}$ without migration of the second proton from the sulfur to the gold atom (TS5). (Figure 40) Askerka et al. compared the two proposed reaction pathways in terms of their hypothetical energy barriers and found that path (b) is lower by $2.7 \mathrm{kcal} \mathrm{mol}^{-1}$, they also noted that all of the elementary stages of this pathway would be exothermic which means that route (b) is more probable than route (a). ${ }^{52}$ Following path (b), it is of interest to note the initial lack of symmetry in comparison to pathway (a). As the Au-H and S-H bonds lengthen by $\sim 0.4$ $\AA$ and $0.1 \AA$ respectively the protons move closer together in preparation for the $\mathrm{H}_{2}$ formation. This transition state requires the system to overcome an energy barrier of $10.2 \mathrm{kcal} \mathrm{mol}^{-1}$ to reach the final product with $\mathrm{C}_{2}$ symmetry. While a single gold atom is not particularly representative of the electronic and energetic properties of an entire gold surface, it serves as an approximation model of a surface atom on an $\mathrm{Au}(111)$ surface and is computationally less expensive.

\subsubsection{Reduction and Oxidation}

It can be seen in the computational study that as the thiols bind to the gold, the sulfur-bound hydrogens are removed and replaced by gold, therefore reducing the nanoparticles. This being the case, it is likely that any surface contaminant would need to be reduced in order to be properly removed from the gold, or that the gold needs to be oxidised in order to 'reset' the surface for further thiol binding. It was possible that as the gold-sulfur bond is very strong, some thiols may remain on the fibres after the 'desorption' step of the protocol and therefore cleaning the fibres after each use may be appropriate to give suitable reproducibility.

\subsubsection{Thiol}

Theoretical research by Andreoni and co-workers investigated the interaction between gold and the head groups of thiols and disulfides. ${ }^{53}$ They demonstrated that the potential for adsorption of both thiols and thiolates to a $\mathrm{Au}(111)$ surface is possible, either releasing molecular hydrogen or with proton binding to the gold, however, the thiolate adsorption is favoured, by a predicted $3 \mathrm{kcal} \mathrm{mol}^{-1}$. This would mean that the two adsorbates may 
exist on the surface at the same time. ${ }^{53}$ They noted that in spite of the predicted similarities in binding energies, the bonds to the surface were very different. In the case of the thiol, there is weak chemisorption whereas the thiolates form two strong chemisorption interactions with the surface. The properties of a disulphide bond to the surface was investigated as it is possible that once the thiol/ates are bound to the gold surface, they dimerise even though theoretically it is energetically favourable for the disulfide to dissociate to two thiolates adsorbed to the surface (by $22 \mathrm{kcal} \mathrm{mol}^{-1}$ ). ${ }^{53}$

With this data, the current research explored the use of hydrogen exposure to the fibre, and alternatively the addition of a strong reducing agent, in an attempt to reduce the bound thiolates to the weakly bound thiols thus facilitating the removal of any residual compounds still bound to the gold after desorption. The effect this had on the reproducibility of the GC-MS results was then examined. The other option that was explored was the oxidation of the thiolates on the surface to disulfides. Andreoni and coworkers discovered that in comparison to the thiolates, the theoretical energy difference between bound and unbound disulfides is $\sim 10 \mathrm{kcal} \mathrm{mol}^{-1}$ lower. ${ }^{53}$

\subsubsection{Gold}

Oxidation of the gold-thiol interaction was thought to prove beneficial in terms of 'resetting' the gold nanoparticles. Previous research has typically indicated that surface oxidation of the gold or exposure to reactive oxygen species may be beneficial to clean the gold coated fibres. Gold cleaning solutions include immersion in $50^{\circ} \mathrm{C}$ sulfochromic acid (saturated $\mathrm{K}_{2} \mathrm{Cr}_{2} \mathrm{O}_{7}$ in conc. $\mathrm{H}_{2} \mathrm{SO}_{4}$ ), $3 \%$ aqueous $\mathrm{HF},{ }^{54}$ immersion in hot concentrated nitric acid, ${ }^{55}$ and immersion in piranha solution $\left(\mathrm{H}_{2} \mathrm{SO}_{4}\right.$ in $\left.\mathrm{H}_{2} \mathrm{O}_{2} 30: 70 \mathrm{v} / \mathrm{v}\right),{ }^{56}$ An example of these is piranha solution, which can be used to clean organic residues off substrates as it is a strong oxidising agent. The fibres could be immersed in the solution for up to 30 minutes and then dried with a stream of nitrogen. However, if the fibres are immersed in the solution for too long, the coating may 'roughen' thus causing a possible degradation of the fibres. ${ }^{56}$

Alternatively, gold surfaces could be subjected to a low pressure quartz-mercury vapour lamp which emits in the $254 \mathrm{~nm}$ to $185 \mathrm{~nm}$ range for about 10 minutes in order to remove some contaminants. This can then be followed by treatment with ozone (which is produced via $\mathrm{UV}$-induced reactions of $\mathrm{O}_{2}$ ) and subsequent immersion in ethanol for 20 minutes. ${ }^{57}$ Another cleaning protocol uses exposure to mild $\mathrm{O}_{2}$ plasma for approximately two 
minutes which may be beneficial to clean the fibres but any longer than this may cause the gold to deteriorate as $\mathrm{O}_{2}$ plasma can oxidise the gold surface. ${ }^{57}$

All of the above techniques are all fairly aggressive as gold is seemingly inert, therefore anything that has bound to it is likely to be difficult to remove. Of the methods described, it was unlikely that many of the methods will be beneficial to the project in the long term as they are potentially toxic, dangerous or too specialised for use in many smaller analytical labs and wineries. However, in terms of obtaining proof of concept (selective binding to sulfur and reproducible extraction of thiols from wine), any means of cleaning the fibres was thought to be a significant advancement in the development of this technique. Of the above cleaning protocols, it was thought that oxidation via $\mathrm{O}_{2}$ plasma or dilute $\mathrm{H}_{2} \mathrm{O}_{2}$ would provide an initial idea of the plausibility of the protocol.

\subsubsection{Procedural Considerations}

In order to look at potential cleaning methods, the procedure of analysis needed to be modified in order to maximise efficiency. There was the potential to introduce methods of cleaning the fibre in this procedure, however, these needed to be incorporated so that a dove-tailed protocol could still be adhered (Figure 42).

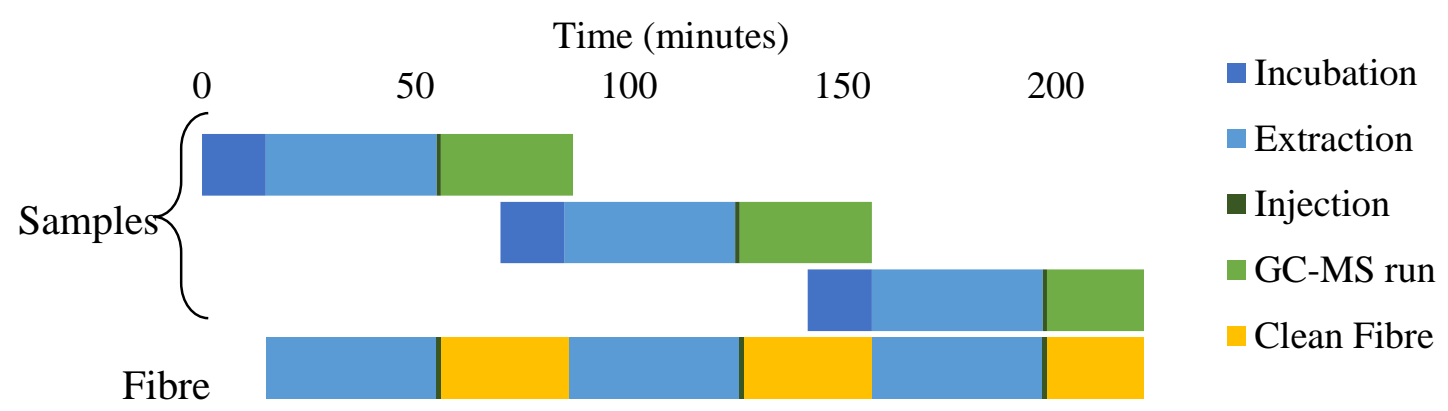

Figure 42: Potential to implement cleaning regime.

While adding a cleaning method to the regime does increase the amount of time taken to analyse the samples it was thought to be necessary in order to 'reset' the fibre for further, more reproducible analyses and only increased the length of time by approximately 30 minutes per sample. 


\subsection{Results and Discussion}

\subsubsection{Computational Chemistry}

Computational analysis of these systems focussed primarily on $3 \mathrm{SH}$ as this was the model compound used for all experimental work. Using a Mulliken population analysis of the starting materials and products allowed comparison of the partial charge differences of atoms in the system (the elementary charge of the atoms is denoted as ' $\overline{\mathrm{e}}$ ' and relates to the asymmetric distribution of electrons in chemical bonds). A comparison of the data collected before and after gold binding to $3 \mathrm{SH}$ is presented in Table 7. It was predicted that as the thiol binds to the gold atom, the charge on the sulfur and many of the carbons decreases, this can also be seen in terms of the variation in shading in Figure 43 where the bright green indicates a more positive partial charge on an atom and conversely, bright red shows more negative partially charged species.

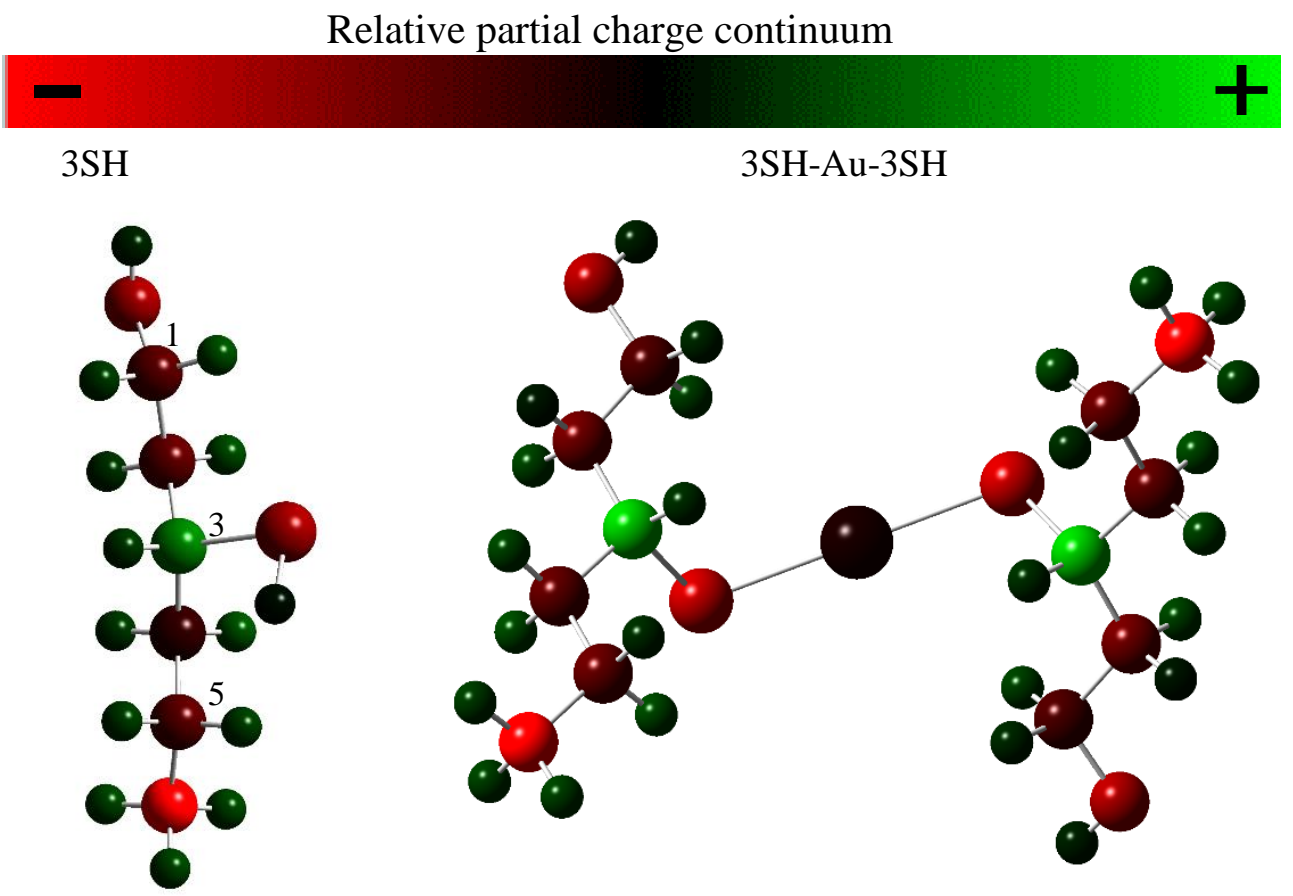

Figure 43: Partial charges of 3SH and 2(3SH)Au.

In the predicted model, the gold brings in an excess of electrons to the complex and in binding to the sulfur, these electrons are, to some extent, involved in the electronic distribution within the complex. Gold is much less electronegative than sulfur and therefore, electrons are drawn towards the sulfur, as seen in the increase of partial 
negative charge on the sulfur atom. The gold has more electrons to contribute to the sulfur than the binding carbon and therefore, the sulfur does not pull as strongly on the carbon's electrons, thus increasing the predicted partial positive charge on the carbon. The predicted bond lengths throughout the molecule remained unchanged for the most part, it was only near the site of attachment (where the largest charge differences occurred) that they were observed to increase very slightly and the $\mathrm{OH}$ bond decreased slightly. Further analysis using natural bonding orbitals and Wiberg indices would further benefit this area of research; however, due to limitations (basis set, functional used and time constraints) these calculations were not available for this research.

Table 7: Partial Charges and Bond Lengths.

\begin{tabular}{|c|c|c|c|}
\hline \multicolumn{2}{|c|}{ 3SH } & \multicolumn{2}{|c|}{ 2(3SH)-Au } \\
\hline Atom & 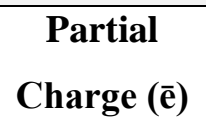 & 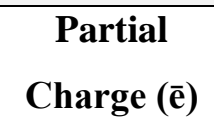 & Difference \\
\hline $\bar{S}$ & -0.51 & "-0.67 & "-0.16 \\
\hline $\mathbf{C}_{1}$ & -0.28 & -0.26 & 0.02 \\
\hline $\mathrm{C}_{2}$ & -0.30 & -0.37 & -0.07 \\
\hline $\mathrm{C}_{3}$ & 0.41 & 0.68 & 0.27 \\
\hline $\mathrm{C}_{4}$ & -0.17 & -0.32 & -0.15 \\
\hline $\mathrm{C}_{5}$ & -0.29 & -0.37 & -0.08 \\
\hline $\mathrm{C}_{6}$ & -0.93 & -0.99 & -0.06 \\
\hline $\mathbf{O}$ & -0.51 & -0.58 & -0.07 \\
\hline $\mathbf{H}_{\mathbf{S}}$ & 0.04 & N/A & N/A \\
\hline $\mathbf{H}_{\mathbf{O}}$ & 0.11 & 0.11 & 0 \\
\hline $\mathbf{H}_{\mathrm{C}}$ & 0.17 & 0.16 & -0.01 \\
\hline Bond & $\begin{array}{c}\text { Bond } \\
\text { Length (̊̊) }\end{array}$ & $\begin{array}{c}\text { Bond } \\
\text { Length }(\AA)\end{array}$ & Difference \\
\hline $\bar{~} \overline{\mathrm{C}_{2}-\mathrm{C}_{3}}$ & 1.53 & 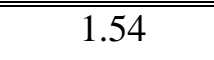 & 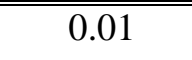 \\
\hline $\mathrm{C}_{3}-\mathrm{C}_{4}$ & 1.53 & 1.54 & 0.01 \\
\hline O-H & 0.97 & 0.95 & -0.02 \\
\hline
\end{tabular}


Once the reactants and products had been optimised it was possible to attempt to compare this reaction with that proposed in previous literature $^{52}$ and the prediction of the mechanism by which the thiols bind to the gold atoms was attempted. With the expectation that a synchronous transit algorithm (Figure 44) between known intermediate configurations could be used to extrapolate desired geometries of transition states, the intermediates were constructed

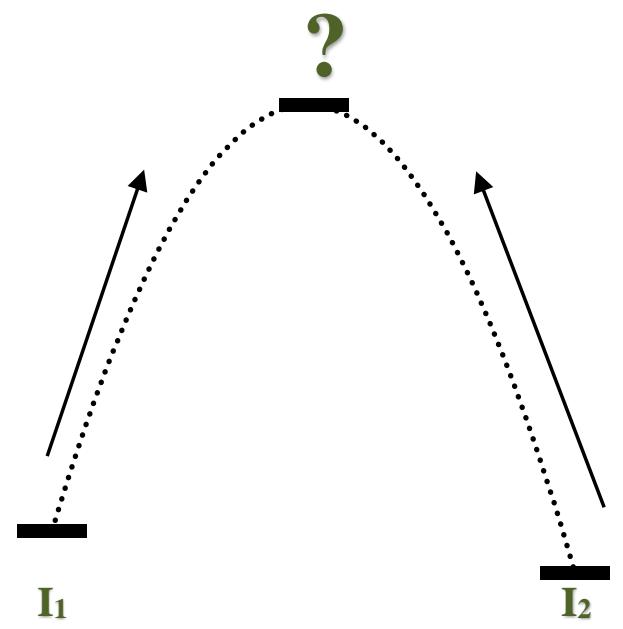
and attempts to optimise them using Figure 44: Method of synchronous transit. Gaussian were pursued. The system, however, is complex in nature and involves a large number of atoms, electrons and therefore interactions that must be accounted for, thus making computational analysis a long, difficult process. In correlation with the Askerka study, ${ }^{52}$ Intermediates 1 and 3 were optimised (Figure 45).
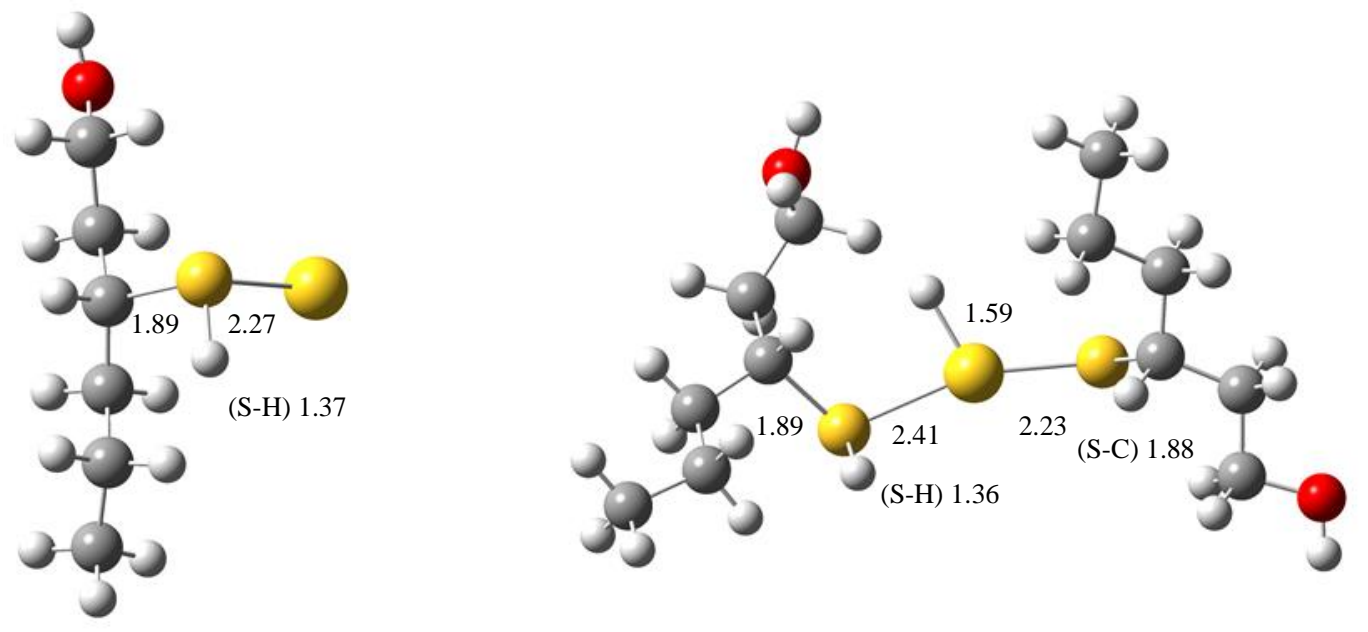

Figure 45: Intermediates 1 and 3 of proposed binding mechanism.

The absolute values of the current study's modelled structures cannot be directly compared to that reported in the literature as minor changes to parameters used in calculations can cause different overall values. However, general trends can be observed and analysed. This research found that the trends seen in the change from $I_{1}$ to $I_{3}$ are very similar to that observed in previous research. ${ }^{52}$ For example, it is predicted that the C-S 
and $\mathrm{S}-\mathrm{H}$ bonds do not significantly change however, in both studies the modelled $\mathrm{Au}-\mathrm{S}$ bonds lengthen and an asymmetry can be observed about the gold atom in $\mathrm{I}_{3}$.

The other intermediates, however, were not as easily optimised. This meant that the transition-states were not able to be optimised or identified either. Future work in this area would be beneficial in gaining a better knowledge of the energy required for the reaction to occur, and hence the energy needed to potentially be reversed. However, it was possible to predict the energy change over the course of the reaction and this was estimated to be $-1410 \mathrm{~kJ} \mathrm{~mol}^{-1}$ showing that the proposed product of a gold atom bound to two thiolates releasing hydrogen gas was significantly more favoured than the gold atom and thiols separate as the reactants as this release of energy is very large. However, this is just a proposed mechanism and the values predicted are estimates with no experimental data is implied.

\subsubsection{Reductions}

Even though the mechanisms were not

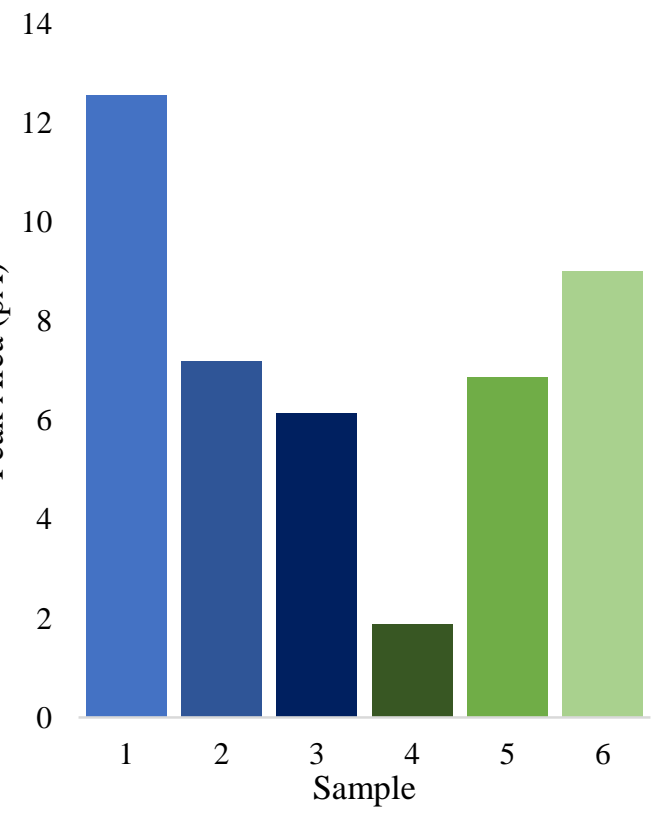

Figure 46: Peak area of $3 \mathrm{SH}$ over subsequent injections to the GC-FID.

in Figure 46. It can be seen in these traces

that the $\mathrm{H}_{2}$ atmosphere did not provide better reproducibilty between runs as the peak area still fluctuated as the experiment progressed. To this end, it was hypothesised that a reductive dip may be a more direct method of reducing the thiol to ensure the gold surface was 'renewed' for the next extraction. Three reductive dips were considered; TSC, hydrazine and $\mathrm{NaBH}_{4}$. The results of which are presented in Figure 47 (only three samples of $\mathrm{NaBH}_{4}$ were run due to time and reductant constraints). For the purpose of reproducible techniques in small scale labs and wineries, mild, non-toxic techniques (such as TSC) 
was prefered, however, it was found that all reductive dips were ineffective in generating reproducibility in this technique. It can be seen that in the case of hydrazine and $\mathrm{NaBH}_{4}$ the peak areas increase over subsequent injections, however, their peak areas (data not shown) were considerably deformed with each run.

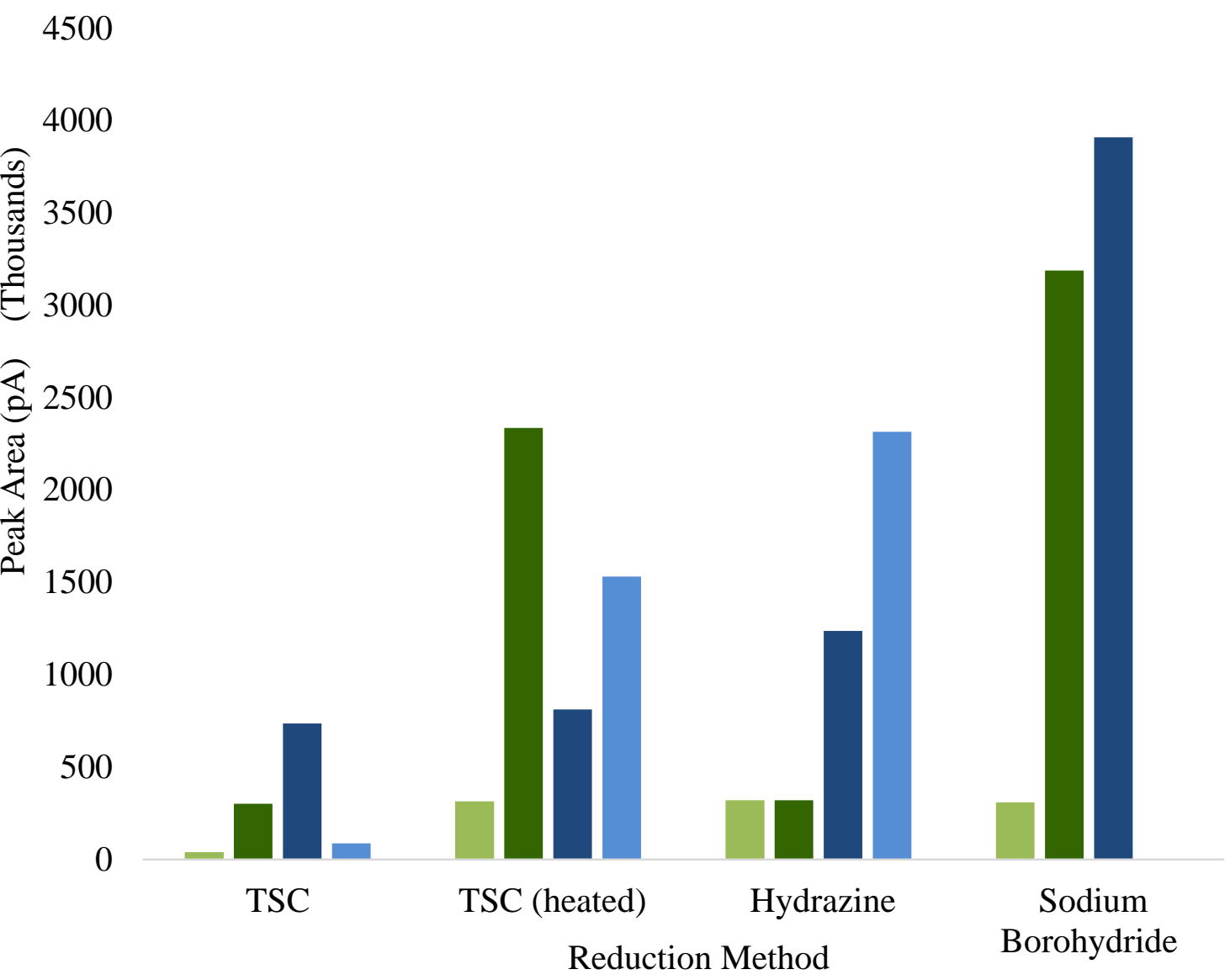

Figure 47: Comparison of the effects of reductive conditions on thiol analysis using GC-MS.

\subsubsection{Oxidation}

An initial test of the possibility of oxidising the fibres to clean them was conducted using thin glass fibres, coated in gold nanoparticles and subsequently adsorbed with thiols which were then desorbed by heat. Oxygen plasma was used to try and remove any residual thiols by oxidising the gold. It could be seen by EDS (data not shown) that the plasma cleaning did not completely destroy the fibres or dislodge all the particles, however, due to the overlap of the sulfur and gold on the EDS analysis it was impossible to determine the effect on the removal of thiols. This technique was not attempted on the SPME fibres as it was thought to be too time consuming and specialised for industrial use.

Hydrogen peroxide in combination with strong acids has been able to clean gold in previous research, ${ }^{56}$ however, in the hope that a milder method might be able to 'reset' 
the gold fibres, a $30 \%(\mathrm{v} / \mathrm{v}) \mathrm{H}_{2} \mathrm{O}_{2}$ was used. After desorption in the GC-MS, the fibre was immersed in the $\mathrm{H}_{2} \mathrm{O}_{2}$ solution for 30 minutes and rinsed with water prior to each extraction. The resulting spectra shows that as with previous experiments, the $3 \mathrm{SH}$ peak at $\sim 9$ minutes fluctuates in area and deforms with subsequent injections (Figure 48 \& Figure 50). However, it is also noted that the peaks between 10.25 minutes and 11 minutes increase in intensity and breadth (Figure 48) which is likely to be due to a phase mismatch in polarity between the non-polar stationary phase and an unexpected polar analyte.

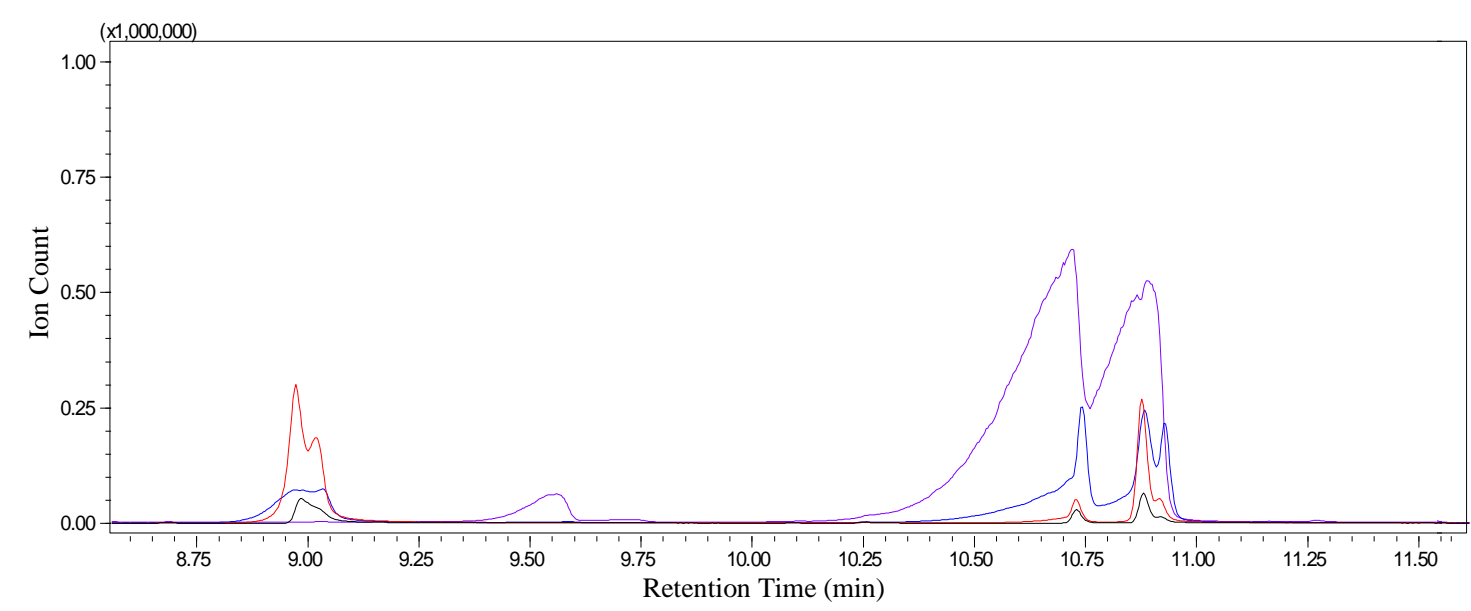

Figure 48: Overlay of HS Chromatograms with oxidation between each injection (8.5 min to $12 \mathrm{~min}$ ) (sample 1: black trace, 2: red, 3: blue and 4: purple).

Later in the chromatograph, it can also be seen that there is an increase in the intensity of three peaks that appear to be correlated with the order in which they were run, which in turn is related to the amount of oxidisation that the fibre and bound compounds encountered. (Figure $49 \&$ Figure 50). This suggested that the fibre oxidation is rendering new products that are becoming more facile to desorb.

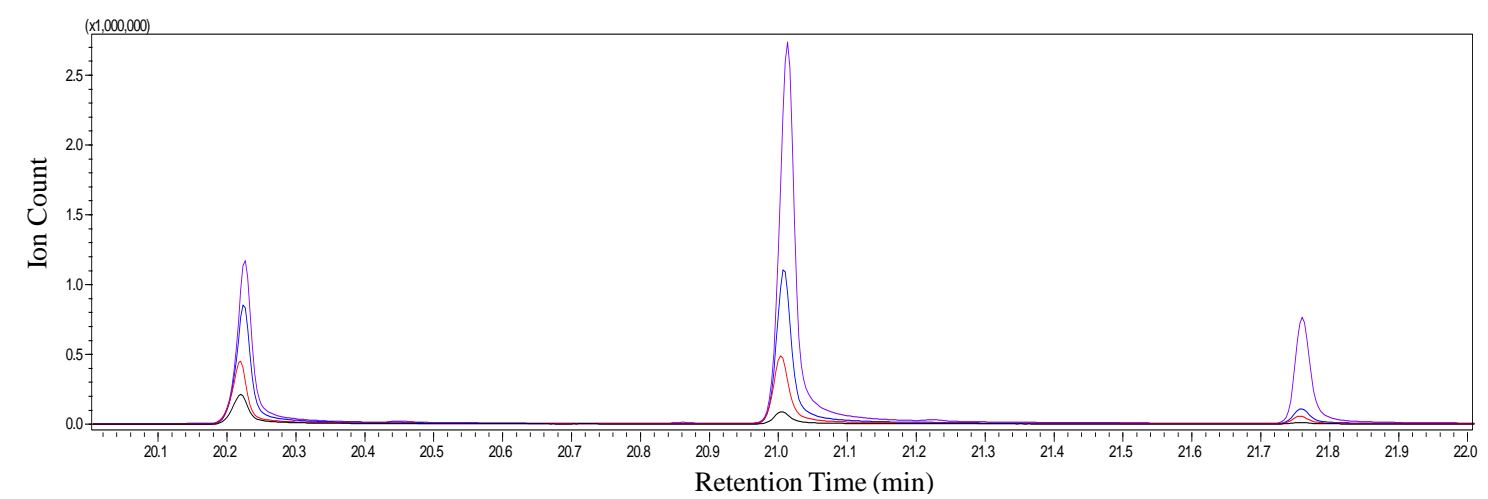

Figure 49: Overlay of HS Chromatograms with oxidation between each injection (20 $\mathrm{min}$ to $22 \mathrm{~min}$ ) (sample 1: black trace, 2: red, 3: blue and 4: purple). 


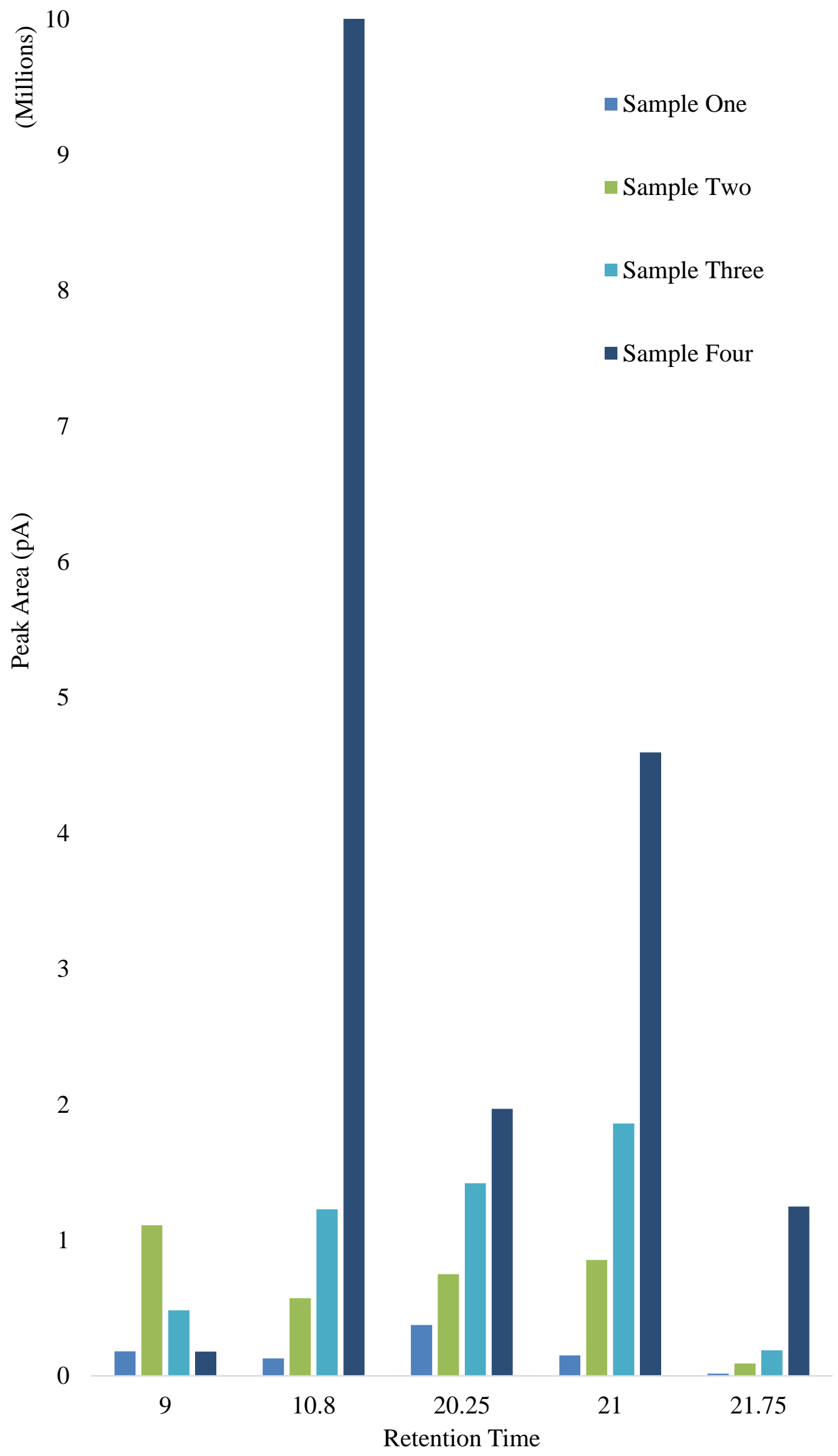

Figure 50: 3SH peak areas of subsequent oxidations. 
These analyses were run in SIM mode, however, with the appearance of new peaks around 10 and 20 minutes the analysis was changed to scan mode in order to better interpret the MS fragmentation patterns to identify these compounds. The peaks of interest were cross-matched with the MS fragmentation patterns in reference libraries, unfortunately, tentative identifications were not found. However, some consistent $\mathrm{m} / \mathrm{z}$ values were seen throughout many of the fragmentation patterns $(\mathrm{m} / \mathrm{z} 55,83,101,115 \&$ 148). It was thought that the fragmentation patterns obtained could be further analysed to give a better idea of what was occurring during the oxidative dip process.

To identify the compounds at each retention time, 3SH and 3SHA were analysed in terms of the potential fragments that they could form. Many of the MS fragmentation patterns for the compounds detected around 20 minutes contained fragment with a parent fragments greater than 134 (monoisotopic mass of $3 \mathrm{SH}$ ), indicating that during the cleaning of the fibre with $\mathrm{H}_{2} \mathrm{O}_{2}$, the thiols had been oxidised to form a disulphide linkage between two thiols (3SH and/or 3SHA - Figure 51) or that they may have been oxidised to a carboxylic acid. Therefore, the simple $3 \mathrm{SH}$ and 3SHA frameworks were used for initial calculations of possible fragments.

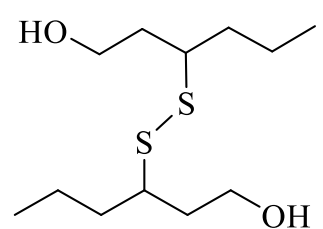

$\mathrm{M}_{\mathrm{r}}: 266$<smiles>CCCC(CCOC(C)=O)SSC(CCC)CCOC(C)=O</smiles>

$\mathrm{M}_{\mathrm{r}}: 350$<smiles>CCCC(CCO)SSC(CCC)CCOC(C)=O</smiles>

$\mathrm{M}_{\mathrm{r}}: 308$

Figure 51: Possible dimers 3SH and 3SHA.

As shown in Figure 48, the compounds found at 10.75 and 10.85 minutes merge together over subsequent runs, analysis of their MS fragmentation patterns indicated that they were very likely to be the same compound. The MS fragmentation pattern for these peaks is shown in Figure 52. 


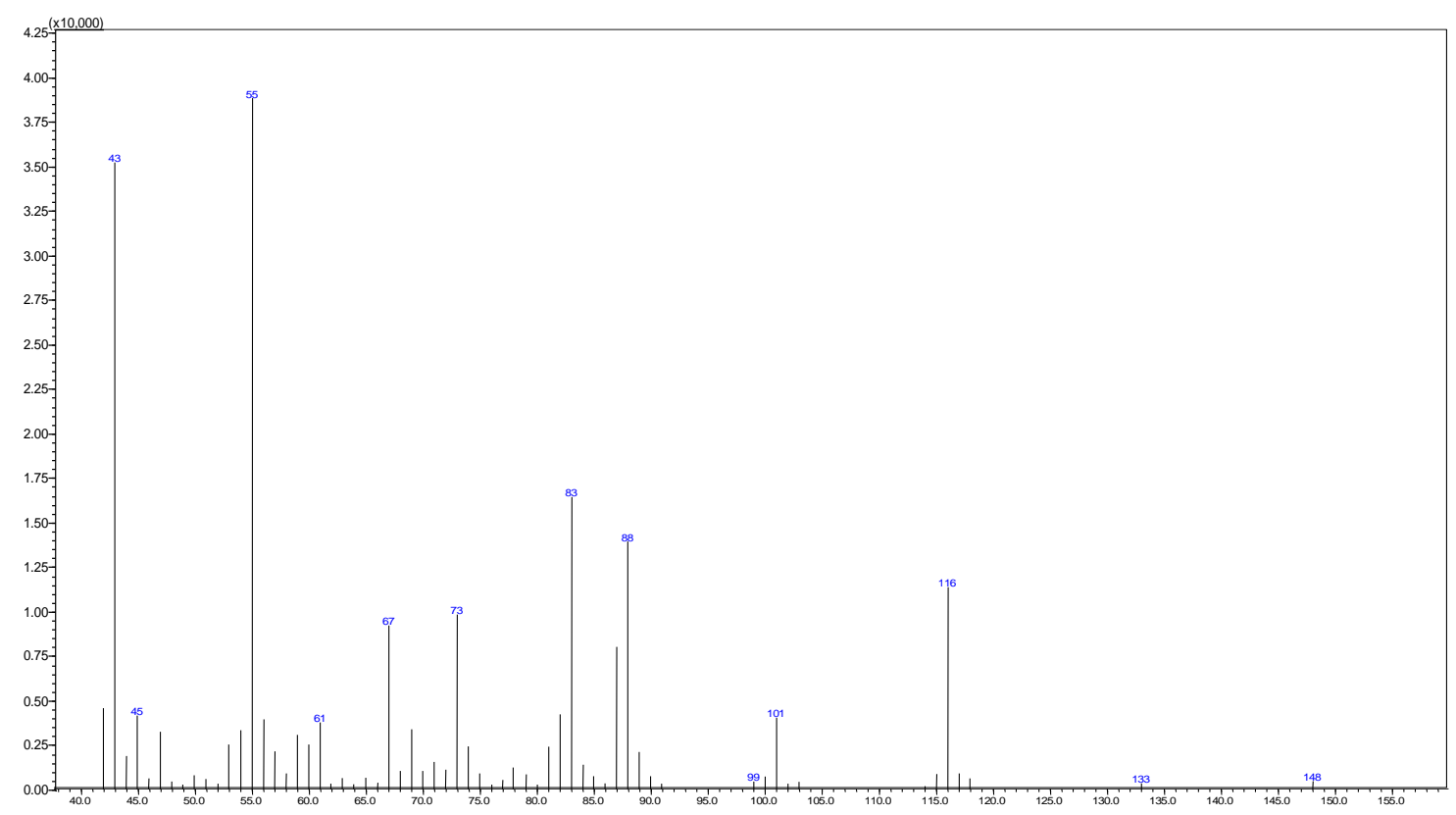

Figure 52: Mass spectral fragmentation pattern for compound eluted at 10.80 minutes.

The $\mathrm{M}+$ peak is at $\mathrm{m} / \mathrm{z} 148$, which is higher than the parent monoisotopic mass of $3 \mathrm{SH}$ by 14 AMU, suggesting an oxidation. This indicated that a chemical reaction had taken place during the oxidation of the fibre. It is hypothesised that if there was still thiol attached to the gold nanoparticles after the desorption phase of the protocol, the alcohol functional group of $3 \mathrm{SH}$, when subjected to $\mathrm{H}_{2} \mathrm{O}_{2}$ was oxidised to its corresponding carboxylic acid (Scheme 7). The monoisotopic mass of 3-sulfanylhexanoic acid is 148 AMU and therefore is likely to be cause of this peak.<smiles>CCCC(S)CCO</smiles>

Scheme 7: Possible oxidation of $3 \mathrm{SH}$ by $\mathrm{H}_{2} \mathrm{O}_{2}$ to form a carboxylic acid.

In a similar manner to the well-known elimination of water fragmentation, the thiol functional group can react with a nearby hydrogen to from the small neutral molecule $\mathrm{H}_{2} \mathrm{~S}$. This reaction leaves behind an alkenyl fragment (Scheme 8) with potential $\mathrm{m} / \mathrm{z}$ values of $41,55,69$, etc.(increasing in 14AMU) which are often greater than or equal in intensity to the alkyl fragments of linear organic molecules. ${ }^{58}$ This is further supports hypothesis that this compound contains some $3 \mathrm{SH}$ functionality.

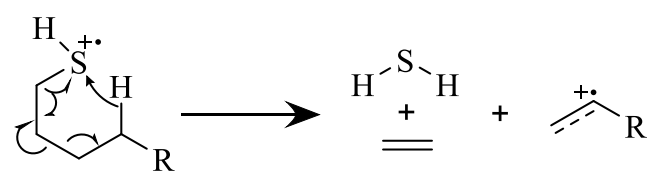

Scheme 8: Fragmentation of linear thiol to give an $\mathrm{M}-\mathrm{H}_{2} \mathrm{~S}-\left(\mathrm{CH}_{2}=\mathrm{CH}_{2}\right)$ series of peaks. 
The loss of a propyl fragment is indicated by a large peak at $\mathrm{m} / \mathrm{z} 43$ and there is a characteristic loss of $\mathrm{COOH}$ to give a small peak at $\mathrm{m} / \mathrm{z} 103$. It is also possible to see the loss of water followed by fragments of the alkyl chain and acid functional group ( $\mathrm{m} / \mathrm{z} 88)$.

The MS fragmentation pattern for the peak at 20.25 minutes is shown in Figure 53. The $\mathrm{M}+$ peak of this compound appears to be at $\mathrm{m} / \mathrm{z} 266$, suitable for a disulfide of $3 \mathrm{SH}$, with an ion at $\mathrm{M}+2$ reminiscent of the low abundance ${ }^{34} \mathrm{~S}$ isotopomer. Primarily, it can be noted that the alkenyl peaks ( $55 \& 83$ ) are present in abundance. The alcohol at the terminus of the thiol can go through an elimination reaction with the neighbouring methylene to afford water (small neutral molecule loss), leaving an alkene at the end of the carbon chain giving an ion at $\mathrm{m} / \mathrm{z} 115$.

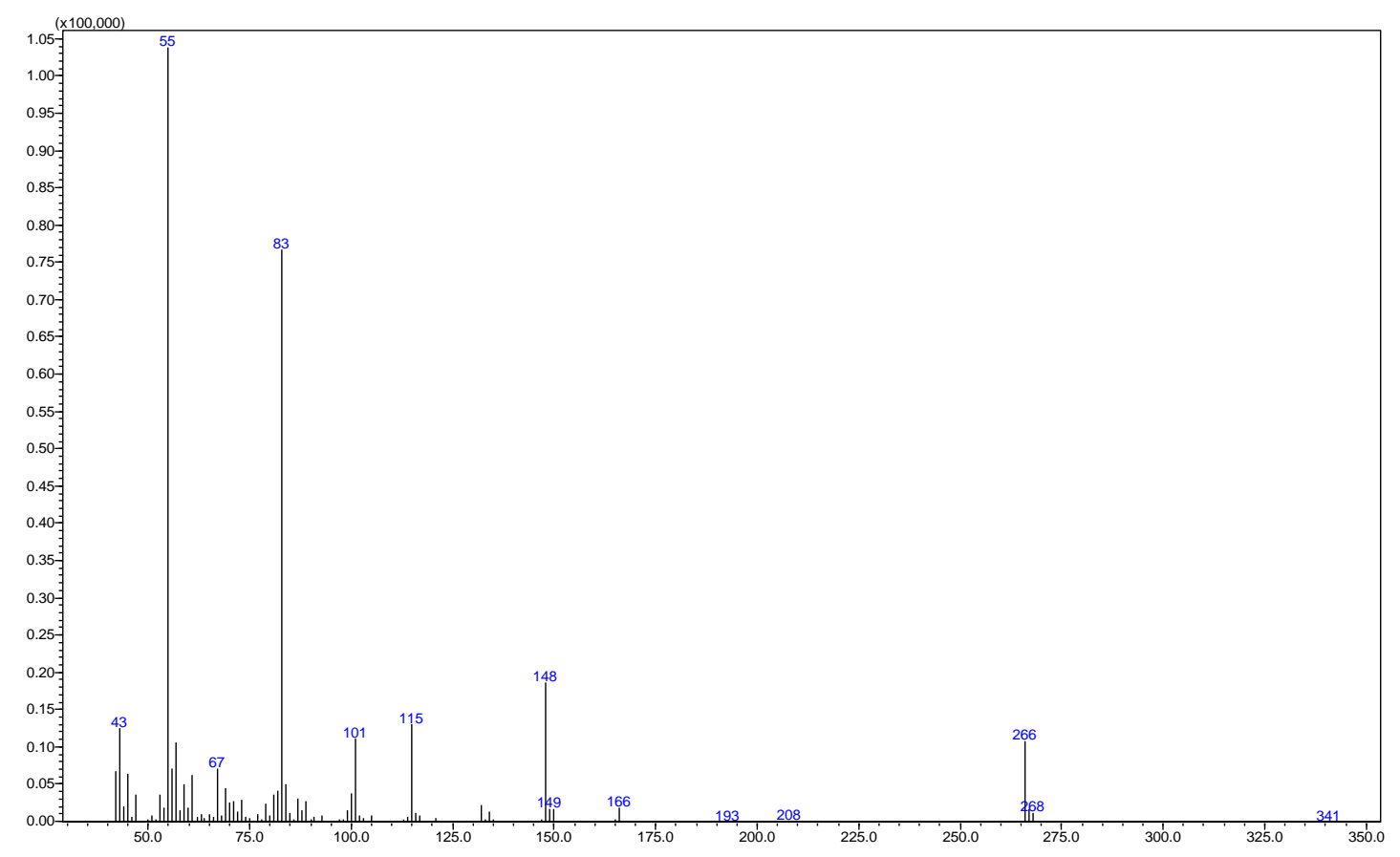

Figure 53: Mass spectral fragmentation pattern for compound eluted at 20.25 minutes

Another fragment ion noted at $\mathrm{m} / \mathrm{z} 101$ is from thiol loss from $3 \mathrm{SH}$ indicating that the compound is likely to contain a $3 \mathrm{SH}$ component. This points towards a $3 \mathrm{SH}$ dimer (Figure 51) as the compound responsible for this MS fragmentation.<smiles>CCCCCCCCC([Si])CCO</smiles>

Scheme 9: Fragmentation of 3SH, loss of thiol. 
Striking similarities can be seen in the MS fragmentation pattern for the compound at 21 minutes, which is demonstrated in Figure 54. However, from the intensity of the ion at $\mathrm{m} / \mathrm{z} 43$, this ion is no longer only accounting for alkyl chain fragmentation but also for the loss of an acetate group. The typical alkenyl fragment series is apparent again and from this the compound is tentatively assigned as a 3SH-3SHA dimer (Figure 51), as 3SHA is present in small quantities as an impurity of the standard solution. Supporting this assumption is the monoisotopic mass of this dimer which is 308 AMU.

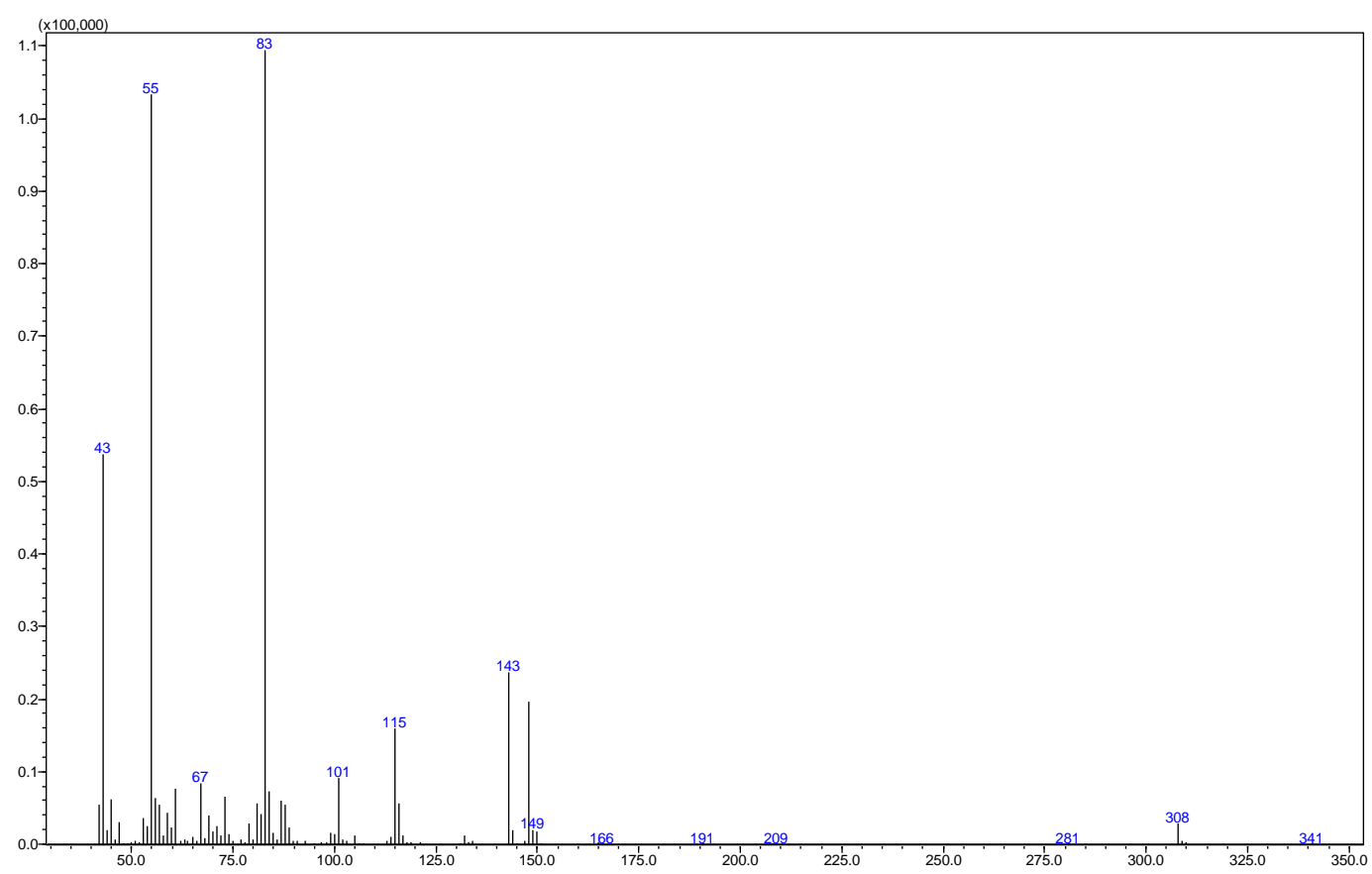

Figure 54: Mass spectral fragmentation pattern for compound eluted at 21 minutes.

The final peak at 21.75 minutes gave the MS fragmentation pattern presented in Figure 55. While the presumed $\mathrm{M}+$ ion is not identifiable in this figure, it is thought that this compound is the 3SHA dimer (Figure 51) that would have a monoisotopic mass of $350 \mathrm{AMU}$ and that this parent ion was too small to be detected. However, noting that the $\mathrm{m} / \mathrm{z} 43$ has significantly increased in size again from the $3 \mathrm{SH}$ dimer and mixed dimer this assumption is further supported as well as the alkenyl peak series and subsequent similarities with the two previous MS fragmentation patterns. 


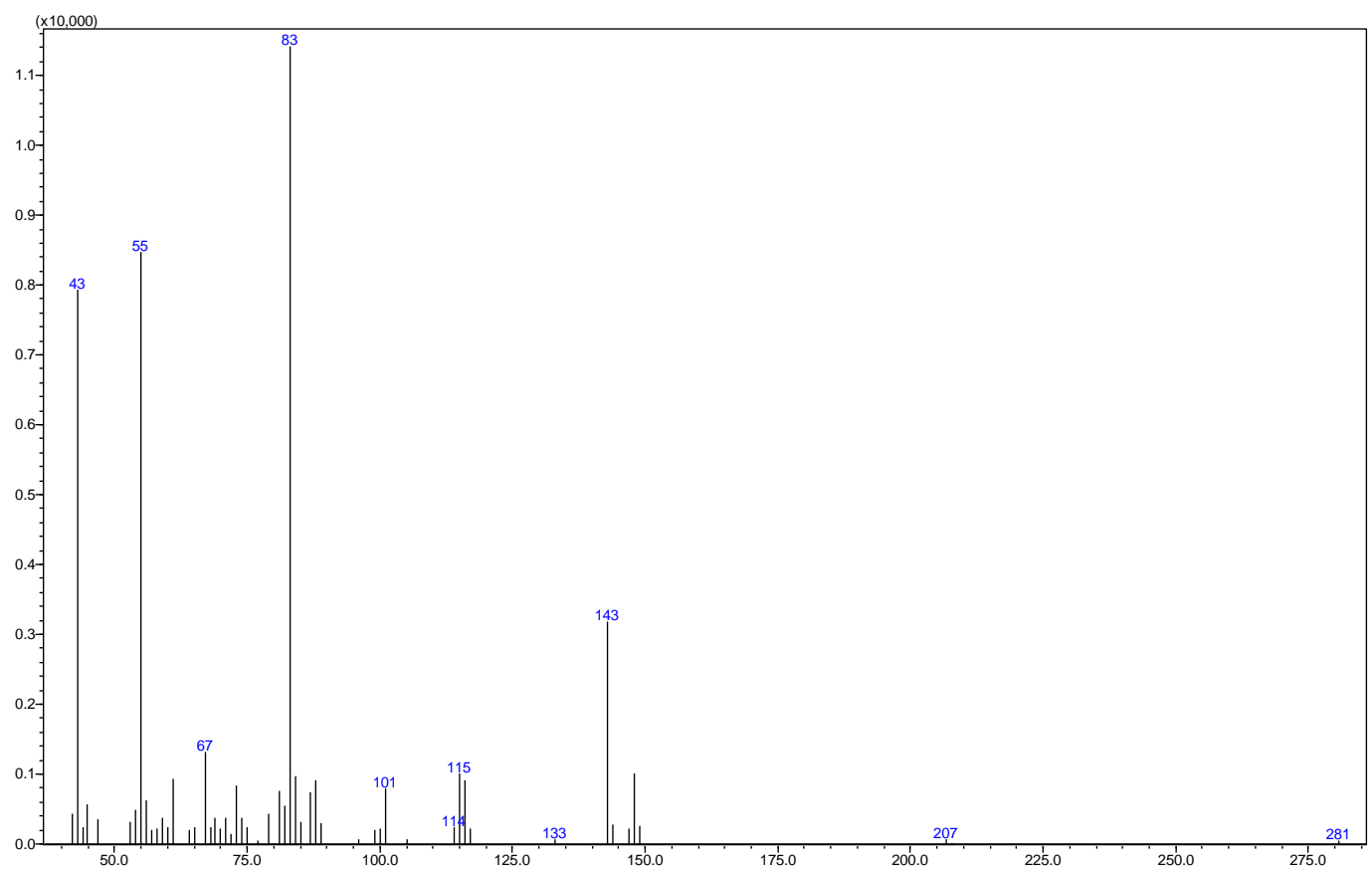

Figure 55: Mass spectral fragmentation pattern for compound eluted at 21.75 minutes

Analysis of the GC peaks after the fibre's oxidative dip, indicates that it is likely that the residual thiolates on the surface of the gold have been oxidised and became easier to displace from the fibre, thus causing a sequential increase of peak area as subsequent injections are analysed. This in turn logically leads to the conclusion that the thiolates had been inadequately desorbed during the analytical process and hence are the source of the “contamination" and hysteresis noted during analysis. Further research should attempt to increase the GC inlet temperature without destroying the fibre or structural integrity of the organic molecules. Attempts should also be made to determine the retention times and MS fragmentation patterns of the proposed disulfide compounds in order to further support these findings. 


\section{Conclusion}

The research described in this thesis has investigated the development of a novel method of analysis for thiols in Sauvignon blanc using gold nanoparticles bound to SPME fibres. The aim was to further develop an analytical protocol that was more reproducible, more accessible and less toxic than the current methods that use organomercury columns or specialised GC techniques. Initially it was discovered that there was a hysteresis effect that cause irreproducibility between samples and this was sought to be overcome by adjusting the kinetic and thermodynamic parameters of the protocol, or by removing potential contaminants. When this area of exploration rendered little advancements the surface interactions of the gold and thiol interface were characterised using microscopy, computational chemistry and SERS. These analyses demonstrated that it was unlikely that external contaminants were the cause of the hysteresis observed and that the binding chemistry of the thiols needed to be further investigated in order to control the adsorption of the thiol to the fibre and during the subsequent desorption. Computational analysis was used in an attempt to map the potential energy surface of the adsorption in order to predict the energy barriers required to break the $\mathrm{Au}-\mathrm{S}$ bond, however due to time constraints this mechanistic pathway remains undefined. For future investigations, this mechanism could be completed in order to determine the transition state energies by which the reaction is predicted to proceed. This information could then be translated to a temperature at which the thiolate-gold bonds will break in a more reliable manner. A natural bonding orbital analysis resulting in Wiberg indices would also provide predicted charge values for each atom and bond strengths which when compared between products and reactants can present predicted electron flow within the molecule and at which step the bonds are likely to be the weakest and therefore more easily broken.

Reduction and oxidation of the system was also investigated and while neither reduction or $\mathrm{O}_{2}$ plasma cleaning induced reproducibility between samples, oxidation with hydrogen peroxide appears to oxidise the thiolates still bound to the gold surface after the desorption. This oxidation is thought to have afforded 3-sulfanylhexanoic acid, and three thiol dimers as disulfide products (tentatively identified by MS fragmentation analysis). This being the case, it would appear that the weaker disulfide-gold bond is broken during the desorption in the GC in a much more facile manner than the thiolate-gold bond. For further development of this technique, it would be pertinent to explore the oxidation of the thiolates further and establish a quantification of the percentage of thiol that is 
desorbed prior to oxidation and then how many oxidation steps it takes to remove all of the thiol. An analysis of the ratio of the thiol forming the $3 \mathrm{SH}$ dimer and contributing to the mixed dimer would also prove interesting as it may be possible to oxidise the thiols prior to desorption, and therefore use quantification of the dimers instead of the thiols themselves.

Alternatively, without using oxidative techniques, it is possible that recoating the fibre with gold nanoparticles between each injection could prove the most ecofriendly technique. A $1 \mathrm{~cm}$, uncoated SPME fibre costs roughly $\$ 300$, and has an estimated surface area of $0.126 \mathrm{~cm}^{2}$. A coating of gold nanoparticles $(\sim 20 \mathrm{~nm}$ diameter $)$ required $3.218 \times 10^{-5} \mathrm{~g}$ of gold which equates to $1.06 \times 10^{-4} \mathrm{~g}$ of $\mathrm{HAuCl}_{4} .3 \mathrm{H}_{2} \mathrm{O}$. $\mathrm{HAuCl}_{4}$ is available for approximately $\$ 200$ per gram and SPME fibres are sold in packs of three for approximately $\$ 900$. Each fibre, when handled carefully could be used in up to 500 analyses. This means that including the cost of APTMS and TSC, each injection of sample would cost approximately $\$ 0.64$. For this alternative protocol to be effective, a method of removing the gold from the fibre without damaging the fibre itself would need to be developed, and the cost of this method would need to minimised. In the attempts of further reducing the cost of the technique, the gold could then be recycled and reused to coat further SPME fibres. Future research will look at the feasibly of re-coating fibres (primarily to explore its reproducibility), how many injections a fibre can withstand in this manner and the subsequent cost of doing so.

Sauvignon blanc currently makes up almost $85 \%$ of the 170 million litres of wine exported by New Zealand each year and is a promising area of potential increased income. Analysis of the key odorant compounds in the wine gives the potential to discover their biosynthetic pathways and is a step towards tailoring them to make designer wines and further increasing New Zealand's export income. Using environmentally friendly techniques keeps New Zealand's green image intact and this thesis has described some contributions to this area. 


\section{Methodology}

3SH, 3SHA and 4MSP were purchased from Alfa Aesar (Ward Hill, MA). All other chemicals used were purchased from Sigma-Aldrich. All reagents and solvents were used as received, without further purification. Solvents were purchased from Fisher Scientific. Uncoated, $1 \mathrm{~cm}$ SPME fibres were purchased from Supelco. All water specified was MQ triple distilled, deionised (18.2 $\Omega$ ) water unless otherwise stated.

SPME fibres were coated with gold nanoparticles using 3-aminopropyltrimethoxysilane (APTMS) as a polymer binding agent between the fibre and gold nanoparticles. The Turkevitch method ${ }^{59}$ of gold nanoparticle synthesis (reducing hydrogen tetrachloroaurate with trisodium citrate) was used to make colloidal nanoparticles that were the colour of a dark red wine.

APTMS ( $2 \mathrm{~mL}$ ) was added to $20 \mathrm{~mL} \mathrm{MeOH}$ in a glass sample vial. The solution was then shaken, left for 10 minutes, and subsequently discarded. An identical solution was then made up in the same vial. To prepare the gold nanoparticles, $0.03 \mathrm{~g}$ of $\mathrm{HAuCl}_{4} .3 \mathrm{H}_{2} \mathrm{O}$ was added to a $100 \mathrm{~mL}$ conical flask and mixed with a stirring bar. $\mathrm{H}_{2} \mathrm{O}(50 \mathrm{~mL})$ was then added and the resulting solution was heated to $100^{\circ} \mathrm{C}$. $\mathrm{Na}_{3}$ (citrate) $(0.06 \mathrm{~g})$ was dissolved in $5 \mathrm{~mL}$ of $\mathrm{H}_{2} \mathrm{O}$ and subsequently added to the solution as it began to boil. Once the solution had boiled for a further 15 minutes, it was removed from heat and stirred for a further 15 minutes. The uncoated fibres were immersed in the APTMS/MeOH dip for one hours and then the gold colloidal solution for a further hour. Upon removal from the gold solution, the fibres were red in colour, they were then subjected to conditioning in the GC-MS for 30 minutes, after which they were blue in colour due to surface aggregation of the nanoparticles.

Solutions of $3 \mathrm{SH}$ were made in bulk, (100-200mL) at a concentration of $50 \mu \mathrm{g} \mathrm{L}^{-1}$ unless otherwise specified. When not in use, these solutions were sealed with Parafilm $®$.

The Reductive dips were made up on the day of their use in SPME vials. Due to the varying degree of reactivity and danger associated with the dips they were prepared in different concentrations. A saturated solution of TSC was prepared by dissolving $4.25 \mathrm{~g}$ in $10 \mathrm{~mL}$ of water. Sodium borohydride $(0.2 \mathrm{~g})$ was dissolved in $15 \mathrm{~mL}$ water to give a $0.35 \mathrm{~mol} \mathrm{~L}^{-1}$ solution. A $0.1 \% \mathrm{v} / \mathrm{v}$ solution of Hydrazine was prepared in $10 \mathrm{~mL}$ water. 


\section{GC-MS}

GC-MS was carried out on a Shimadzu (Kyoto, Japan) GC-2010 containing a 30 m Restek (Bellefonte, PA) RTX®-5SilMS crossbond $®$ column with $0.25 \mathrm{~mm}$ internal diameter and $0.25 \mu \mathrm{m}$ film thickness attached to a Shimadzu GC-MS QP 2020 Plus El MS (Electron impact at $70 \mathrm{eV}$ detecting $\mathrm{m} / \mathrm{z} 55,67,82,88,100,116,134$ in SIM mode). The software used for data analysis was Shimadzu GCMSsolutions.

Thiol samples ( $10 \mathrm{ml}$ of $50 \mu \mathrm{g} \mathrm{L}^{-1}$ or $60 \mu \mathrm{g} \mathrm{L}^{-1}$ ) were transferred to a $20 \mathrm{~mL}$ headspace vial. Headspace sampling involved an incubation period at $37^{\circ} \mathrm{C}$ and an extraction period where the fibre was exposed to the headspace of the sample. The sample was then manually injected into the GC-FID for a desorption time of 1 minute (injection temperature $150^{\circ} \mathrm{C}$ ). For all analyses, the temperature profile was as follows: $50^{\circ} \mathrm{C}$ for 2 minutes, raised to $300^{\circ} \mathrm{C}$ at $10^{\circ} \mathrm{C}$ per min followed by a $5 \mathrm{~min}$ isotherm. For gas chromatography the helium carrier gas was passed through the column with a column flow rate of $1.2 \mathrm{~mL} \mathrm{~min}^{-1}$. The mass spectrometer was functioning in electron impact mode in positive mode with a source temperature at $230^{\circ} \mathrm{C}$. The thiols of interest were recognised by their retention time and mass spectrometry fragmentation patterns in comparison to known standards.

\section{GC-FID ( $\mathrm{H}_{2}$ carrier gas)}

Analysis was carried out on a Hewlett Packard (Palo Alto, CA) HP 6890 series GC system containing a $30 \mathrm{~m}$ Aglient DB-WAX column with $0.25 \mathrm{~mm}$ internal diameter and 0.25 $\mu \mathrm{m}$ film thickness attached to a flame ionization detector The software for data analysis was GC ChemStation.

Thiol samples $\left(10 \mathrm{ml}\right.$ of $\left.50 \mu \mathrm{g} \mathrm{\textrm {L } ^ { - 1 }}\right)$ were transferred to a $20 \mathrm{~mL}$ headspace vial. Headspace sampling involved an incubation period at $37^{\circ} \mathrm{C}$ and an extraction period where the fibre was exposed to the headspace of the sample. The sample was then manually injected) into the GC-FID for a desorption time of 1 minute (injection temperature $270^{\circ} \mathrm{C}$ ). The sample was injected in splitless mode on a DB-WAX column (50 m x $250 \mu \mathrm{m}$ internal diameter $\mathrm{x} 0.2 \mu \mathrm{m}$ film thickness). For all analyses, the temperature profile was as follows: $50^{\circ} \mathrm{C}$ for 5 minutes, raised to $115^{\circ} \mathrm{C}$ at $3{ }^{\circ} \mathrm{C} \mathrm{min}^{-1}$ and held for 0 minutes, raised to $150^{\circ} \mathrm{C}$ at $40^{\circ} \mathrm{C} \mathrm{min}{ }^{-1}$ and held for 5 minutes, raised to $173^{\circ} \mathrm{C}$ at $3^{\circ} \mathrm{C} \mathrm{min}{ }^{-1}$ and held for $0 \mathrm{~min}$, raised to $230^{\circ} \mathrm{C}$ at $70^{\circ} \mathrm{C} \mathrm{min}-1$ and held for 15 minutes, lowered to $50^{\circ} \mathrm{C}$ at $40^{\circ} \mathrm{C} \mathrm{min}^{-1}$ 
and held for 0 minutes. For gas chromatography the hydrogen carrier gas was passed through the column with a column head pressure of 30.384 psi and a flow rate of 1 $\mathrm{mL} \min ^{-1}$. The thiols of interest were recognised by their retention time and comparison to a standard of (conc) $3 \mathrm{SH}$ in EtOAc.

\section{SERS}

All experiments were done with the $633 \mathrm{nM}$ line of a HeNE laser with $3 \mathrm{~mW}$ at the sample x 20 objective and 600 lines per millimetre were used. A depth of 400uM focus was used. All samples were prepared in MQ water and sealed in capped plastic cuvettes parafilm ${ }^{\circ}$.

\section{Plasma}

Analysis was carried out on a PE-50 Benchtop Plasma Cleaner using $\mathrm{O}_{2}$ at $20 \mathrm{sccm}$. The plasma chamber was cleaned for one minute prior to addition of samples. Gold coated fibres were placed in the chamber on top of a watch glass so they could be moved in and out of the chamber without interference to the surface of the fibre and were exposed to the $\mathrm{O}_{2}$ plasma for between 8 and 20 seconds at a time.

\section{Electron Microscopy}

SEM samples were carbon coated to prevent charging using the JEOL 6500F microscope for secondary electron image, backscattered electron images, energy dispersive spectroscopy and elemental mapping. TEM samples were drop cast onto a copper grid for imaging using the JEOL 2010 microscope. Analysis of images was performed using JEOL Analysis Station.

\section{Computational}

The current study used density functional theory (DFT) with the Becke-Perdew (BP86) density functional to calculate the mechanistic steps of the reaction of a thiol (3SH, 3SHA or 4MSP) binding to a gold atom, the binding energies of these steps were characterized by using correlation consistent triple- $\zeta$ basis sets (aug-cc-pVTZ/aug-cc-pVTZ-PP) for $\mathrm{H}^{60}$, $\mathrm{C}^{61}, \mathrm{O}^{61}, \mathrm{~S}^{61}$, and $\mathrm{Au}^{62,63}$. For $\mathrm{Au}$ a scalar relativistic energy-consistent small-core pseudopotential of the Stuttgart group was used. The nature of all stationary points was examined through the calculation of the second-derivative matrix. For the Raman calculations a DFT (B3LYP) functional was used with a 6-31G basis set with correlation consistent triple- $\zeta$ basis sets (aug-cc-pVTZ/aug-cc-pVTZ-PP) for $\mathrm{H}^{60}, \mathrm{C}^{61}, \mathrm{O}^{61}, \mathrm{~S}^{61}$, and 
$\mathrm{Au}^{62,63}$. For $\mathrm{Au}$ a scalar relativistic energy-consistent small-core pseudopotential of the Stuttgart group was used. The Gaussian09 suite of programs was used for all calculations.

\section{UV-Vis}

Samples of colloidal gold were diluted to a pale pink, translucent solution $\left(3.55 \times 10^{-4} \mathrm{~mol} \mathrm{~L}^{-1}\right)$ and pipetted into quartz cuvettes for analysis using an Agilent 8453 UV-Vis spectrophotometer with a photodiode array, the samples were scanned between 300 and $800 \mathrm{~nm}$.

\section{Zetasizer}

Colloidal gold samples were diluted to a concentration of $3.55 \times 10^{-4} \mathrm{~mol} \mathrm{~L}^{-1}$ and their size and charge were determined using a Malvern zetasizer (Nano ZS) using NonInvasive Backscatter optics. 


\section{References}

1. New Zealand Winegrowers Association Annual Report; New Zealand Winegrowers Association: 2012.

2. New Zealand Winegrowers Association Annual Report; New Zealand Winegrowers Association: 2013.

3. New Zealand Winegrowers Association Annual Report; New Zealand Winegrowers Association: 2010.

4. Whiteman, N. K.; Pierce, N. E., Delicious poison: genetics of Drosophila host plant preference. Trends in Ecology \& Evolution 2008, 23 473-478.

5. Harrington, R. J., Defining Gastronomic Identity: The Impact of Environment and Culture on Prevailing COmponents, Texture and Flavours in Wine and Food. Journal of Culinary Science \& Technology 2005, 4(2/3) 129.

6. Behrens, M.; Meyerhof, W.; Hellfritsch, C.; Hofmann, T., Sweet and umami taste: natural products, their chemosensory targets, and beyond. Angewandte Chemie International Edition 2011, 50 2220-42.

7. Harrington, R. J., The Wine and Food Pairing Process. Journal of Culinary Science \& Technology 2006, 4:1 101-112.

8. $\quad$ Tamura, T.; Taniguchi, K.; Suzuki, Y.; Okubo, T.; Takata, R.; Konno, T., Iron Is an Essential Cause of Fishy Aftertaste Formation in Wine and Seafood Pairing. Journal of Agricultural and Food Chemistry 2009, 57 8550-8556.

9. Dunlevy, J. D.; Kalua, C. M.; Keyzers, R. A.; Boss, P. K., The production of flavour and aroma compounds in grape berries. In Grapevine Molecular Physiology and Biotechnology, 2 ed.; Roubelakis-Angelakis, K. A., Ed. Springer Science+Business Media: 2009.

10. Tominaga, T.; Dubourdieu, D., A Novel Method for Quantification of 2-Methyl3-furanthiol and 2-Furanmethanethiol in Wines Made from Vitis vinifera Grape Varieties. Journal of Agricultural and Food Chemistry 2006, 54 29-33.

11. Green, J. A.; Parr, W. V.; Breitmeyer, J.; Valentin, D.; Sherlock, R., Sensory and chemical characterisation of Sauvignon blanc wine: Influence of source of origin. Food Research International 2011, 44 2788-2797.

12. Hengstler, K. Konrad Wines. http://www.konradwines.co.nz/malborough.html (accessed 01/06/2012).

13. Parr, W. V.; Green, J. A.; White, K. G.; Sherlock, R. R., The distinctive flavour of New Zealand Sauvignon blanc: Sensory characterisation by wine professionals. Food Quality and Preference 2007, 18 849-861.

14. Swiegers, J. H.; Kievit, R. L.; Siebert, T.; Lattey, K. A.; Bramley, B. R.; Francis, I. L.; King, E. S.; Pretorius, I. S., The influence of yeast on the aroma of Sauvignon Blanc wine. Food Microbiology 2009, 26 204-211.

15. Trought, M. C. T.; Agnew, R. A.; Bennett, J. S.; Stronge, K.; Parr, W.; Greven, M. In Soils, Climate and Vine Management: Their Influence on Marlborough Sauvignon blanc wine style, VIII international terroir congress, Soave (VR), Italy, Soave (VR), Italy, 2010.

16. Tesic, D.; Woolley, D. J.; Hewett, E. W.; Martin, D. J., Environmental effects on cv Cabernet Sauvignon (Vitis vinifera L.) grown in Hawke's Bay, New Zealand.: 1. Phenology and characterisation of viticultural environments. Australian Journal of Grape and Wine Research 2002, 8 15-26.

17. Tominaga, T.; Furrer, A.; Henry, R.; Dubourdieu, D., Identification of new volatile thiols in the aroma of Vitis vinifera L. var. Sauvignon blanc wines. Flavour and Fragrance Journal 1998, 13 159-162. 
18. Ferreira, V.; Ortin, N.; Cacho, J. F., Optimization of a procedure for the selective isolation of some powerful aroma thiols. Journal of Chromatography A 2007, 1143 190198.

19. Kennedy, J. Understanding grape berry development 2002. http://www.practicalwinery.com/JulyAugust02/julaug02p14.htm (accessed 05/08/2012). 20. Subileau, M.; Schneider, R.; Salmon, J.-M.; Degryse, E., New insights on 3mercaptohexanol (3MH) biogenesis in Sauvignon Blanc wines: Cys-3MH and (E)-hexen2-al are not the major precursors. Journal of Agricultural and Food Chemistry 2008, 56 9230-9235.

21. Harsch, M. J.; Benkwitz, F.; Frost, A.; Colonna-Ceccaldi, B.; Gardner, R. C.; Salmon, J.-M., New Precursor of 3-Mercaptohexan-1-ol in Grape Juice: Thiol-Forming Potential and Kinetics during Early Stages of Must Fermentation. Journal of Agricultural and Food Chemistry 2013, 61 3703-3713.

22. Analytical Chemistry Laboratory Manual. http://www.cord.edu/dept/chemistry/analyticallabmanual/experiments/gcms/intro.html (accessed 30 August).

23. Kilmartin, P. Personal Communication,2012.

24. Tominaga, T.; Murat, M.-L.; Dubourdieu, D., Development of a Method for Analyzing the Volatile Thiols Involved in the Characteristic Aroma of Wines Made from Vitis vinifera L. Cv. Sauvignon Blanc. Journal of Agricultural and Food Chemistry 1998, 46 1044-1048.

25. Tominaga, T.; Niclass, Y.; Frérot, E.; Dubourdieu, D., Stereoisomeric distribution of 3-mercaptohexan-1-ol and 3-mercaptohexyl acetate in dry and sweet white wines made from vitis vinifera (Var. Sauvignon blanc and Semillion). Journal of Agricultural and Food Chemistry 2006, 54 7251-7255.

26. Sourabié, A. M.; Spinnler, H.-E.; Bonnarme, P.; Saint-Eve, A.; Landaud, S., Identification of a Powerful Aroma Compound in Munster and Camembert Cheeses: Ethyl 3-Mercaptopropionate. Journal of Agricultural and Food Chemistry 2008, 56 46744680 .

27. Mateo-Vivaracho, L.; Ferreira, V.; Cacho, J., Automated analysis of 2-methyl-3furanthiol and 3-mercaptohexyl acetate at ngL-1 level by headspace solid-phase microextraction with on-fibre derivatization and gas chromatography-negative chemical ionization mass spectrometric determination. Journal of Chromatography A 2006, 1121 $1-9$.

28. Mateo-Vivaracho, L.; Zapata, J.; Cacho, J.; Ferreira, V., Analysis, Occurrence, and Potential Sensory Significance of Five Polyfunctional Mercaptans in White Wines. Journal of Agricultural and Food Chemistry 2010, 58 10184-10194.

29. Capone, D. L.; Sefton, M. A.; Jeffery, D. W., Application of a Modified Method for 3-Mercaptohexan-1-ol Determination To Investigate the Relationship between Free Thiol and Related Conjugates in Grape Juice and Wine. Journal of Agricultural and Food Chemistry 2011, 59 4649-4658.

30. Warren, J. M.; Parkinson, D.-R.; Pawliszyn, J., Assessment of Thiol Compounds from Garlic by Automated Headspace Derivatized In-Needle-NTD-GC-MS and Derivatized In-Fiber-SPME-GC-MS. Journal of Agricultural and Food Chemistry 2012, $61492-500$.

31. Damodaran, V. Summer Project Report - Wine thiol analysis; Victoria University of Wellington: 2010.

32. Pawliszyn, J.; Pawliszyn, B.; Pawliszyn, M., Solid phase microextraction (SPME). Journal of Chemical Education 1997, 2.

33. Risticevic, S.; Lord, H.; Gorecki, T.; Arthur, C. L.; Pawliszyn, J., Protocol for solid-phase microextraction method development. Nat. Protocols 2010, 5 122-139. 
34. Risticevic, S.; Chen, Y.; Kudlejova, L.; Vatinno, R.; Baltensperger, B.; Stuff, J. R.; Hein, D.; Pawliszyn, J., Protocol for the development of automated high-throughput SPME-GC methods for the analysis of volatile and semivolatile constituents in wine samples. Nature Protocols 2010, 5 162-176.

35. Yang, X.; Peppard, T., Solid-Phase Microextraction for Flavor Analysis. Journal of Agricultural and Food Chemistry 1994, 42 1925-1930.

36. Ulman, A., Formation and Structure of Self-Assembled Monolayers. Chemical Reviews 1996, 96 1533-1554.

37. Love, J. C.; Estroff, L. A.; Kriebel, J. K.; Nuzzo, R. G.; Whitesides, G. M., SelfAssembled Monolayers of Thiolates on Metals as a Form of Nanotechnology. Chemical Reviews 2005, 105 1103-1169.

38. Häkkinen, H., The Gold-Sulfur interface at the nanoscale. Nature Chemistry 2012, 4 443-455.

39. Bain, C. D.; Troughton, E. B.; Tao, Y. T.; Evall, J.; Whitesides, G. M.; Nuzzo, R. G., Formation of monolayer films by the spontaneous assembly of organic thiols from solution onto gold. Journal of the American Chemical Society 1989, 111 321-35.

40. Low, D. Characterisation of Non-Linear Thiols on Gold; Towards New Detection of Thiols in Wine. Flinders University, Adelaide, Australia, 2010.

41. Weatherall, E. Development of a Novel Method for Thiol Analysis in Sauvignon Blanc; Victoria University of Wellington: 2011.

42. Farr, A.; Greenwood, D. The Evaluation of a Novel Method for Wine Thiol Analysis; University of Auckland: 2008.

43. Bellino, M. G.; Calvo, E. J.; Gordillo, G., Adsorption kinetics of charged thiols on gold nanoparticles. Physical Chemistry Chemical Physics 2004, 6 424-428.

44. NIST11. http://www.nist.gov/.

45. Njoki, P. N.; Lim, I. I. S.; Mott, D.; Park, H.-Y.; Khan, B.; Mishra, S.; Sujakumar, R.; Luo, J.; Zhong, C.-J., Size Correlation of Optical and Spectroscopic Properties for Gold Nanoparticles. The Journal of Physical Chemistry C 2007, 111 14664-14669.

46. Halls, M. D.; Schlegel, H. B., Comparison study of the prediction of Raman intensities using electronic structure methods. The Journal of Chemical Physics 1999, $1118819-8824$.

47. Sanders, J. V., Transmission electron microscopy of catalysts. Journal of Electron Microscopy Technique 1986, 3 67-93.

48. Goldstein, G. I.; Newbury, D. E.; Echlin, P.; Joy, D. C.; Fiori, C.; Lifshin, E., Scanning electron microscopy and x-ray microanalysis. Plenum Press: New York, 1981. 49. LeRu, E. C.; Etchegoin, P. G., Principles of surface-enhanced Raman spectroscopy and related plasmonic effects. Elsevier: Amsterdam, 2009.

50. Lambert, J. B.; Shurvell, H. F.; Lightner, D. A.; Cooks, R. G., Organic Structural Spectroscopy. Prentice-Hall, Inc.: New Jersey, 1998.

51. Swanson, H. E.; Tatge, E., Standard X - Ray Diffraction Powder Patterns. Journal of Research of the National Bureau of Standards 1953, 539.

52. Askerka, M.; Pichugina, D.; Kuz'menko, N.; Shestakov, A., Theroetical Prediction of S-H Bond Rupure in Methanethiol upon Interaction with Gold. The Journal of Physical Chemistry A 2012, 1168.

53. Grönbeck, H.; Curioni, A.; Andreoni, W., Thiols and Disulfides on the Au(111) Surface: The Headgroup-Gold Interaction. Journal of the American Chemical Society 2000, 122 3839-3842.

54. Miller, C.; Cuendet, P.; Graetzel, M., Adsorbed $\omega$-hydroxy thiol monolayers on gold electrodes: evidence for electron tunneling to redox species in solution. Journal of Physical Chemistry 1991, 95 877-86. 
55. Gomez, M.; Li, J.; Kaifer, A. E., Surfactant monolayers on electrode surfaces: self-assembly of a series of amphiphilic viologens on gold and tin oxide. Langmuir 1991, 7 1797-806.

56. Evans, S. D.; Sharma, R.; Ulman, A., Contact angle stability: Reorganization of monolayer surfaces. Langmuir 1991, 7 156-61.

57. Ron, H.; Matlis, S.; Rubinstein, I., Self-Assembled Monolayers on Oxidized Metals. 2. Gold Surface Oxidative Pretreatment, Monolayer Properties, and Depression Formation. Langmuir 1998, 14 1116-1121.

58. Silverstein, R. M.; Webster, F. X.; Kiemle, D. J., Spectrometric Identification of Organic Compounds. 7 ed.; John Wiley \& Sons Inc.: 2005.

59. Turkevich, J.; Stevenson, P. C.; Hillier, J., The nucleation and growth processes in the synthesis of colloidal gold. Discussions of the Faraday Society 1951, No. 11 55-75.

60. Dunning, T. H., Gaussian basis sets for use in correlated molecular calculations. I. The atoms boron through neon and hydrogen. The Journal of Chemical Physics 1989, 90 1007-1023.

61. Woon, D. E.; Dunning, T. H., Gaussian basis sets for use in correlated molecular calculations. III. The atoms aluminum through argon. The Journal of Chemical Physics 1993, 98 1358-1371.

62. Schwerdtfeger, P.; Dolg, M.; Schwarz, W. H. E.; Bowmaker, G. A.; Boyd, P. D. W., Relativistic effects in gold chemistry. I. Diatomic gold compounds. The Journal of Chemical Physics 1989, 91 1762-1774.

63. Peterson, K. A.; Puzzarini, C., Systematically convergent basis sets for transition metals. II. Pseudopotential-based correlation consistent basis sets for the group $11(\mathrm{Cu}$, $\mathrm{Ag}, \mathrm{Au})$ and 12 ( $\mathrm{Zn}, \mathrm{Cd}, \mathrm{Hg}$ ) elements. Theoretical Chemistry Accounts 2005, 114283 296. 